


\section{Propositions}

1. In transportation models, cost minimisation objectives can also reduce emissions if fuel estimations were considered more realistically. (this thesis)

2. Logistics cooperation improves eco-efficiency. (this thesis)

3. If models are your reality, you have a simplified worldview.

4. Involving business in science adds value but it should not be a prerequisite.

5. The government's plan to subsidise companies to come up with their own emission reduction plan should be replaced by a tax on $\mathrm{CO}_{2}$ emissions.

6. Social sustainability is hard to define but easy to improve.

Propositions belonging to the thesis, entitled 'Cooperative food logistics: towards eco-efficiency'

Helena Margaretha Stellingwerf

Wageningen, June 21, 2019 



\section{Cooperative food logistics: towards eco-efficiency}

Helena Margaretha Stellingwerf 


\section{Thesis committee}

\section{Promotor}

Prof. Dr ].M. Bloemhof-Ruwaard

Personal Professor of Operations Research and Logistics

Wageningen University \& Research

Prof. Dr ].G.A.J. van der Vorst

Professor of Logistics and Operations Research

Wageningen University \& Research

\section{Co-promotor}

Dr A. Kanellopoulos

Assistant professor of Operations Research and Logistics

Wageningen University \& Research

\section{Other members}

Prof. Dr M.P.M. Meuwissen, Wageningen University \& Research

Dr E.M.T. Hendrix, University of Malaga, Spain

Dr A. Palmer, Heriot-Watt University, United Kingdom

Prof. DrW.E.H. Dullaert, Vrije Universiteit Amsterdam

This research was conducted under the auspices of the Wageningen School of Social Sciences (WASS). 


\title{
Cooperative food logistics: towards eco-efficiency
}

\author{
Helena Margaretha Stellingwerf
}

\section{Thesis}

submitted in fulfilment of the requirements for the degree of doctor at Wageningen University

by the authority of the Rector Magnificus,

Prof. Dr A.P.J. Mol,

in the presence of the

Thesis Committee appointed by the Academic Board

to be defended in public

on Friday June 21, 2019

at 4 p.m. in the Aula. 
H.M. Stellingwerf

Cooperative food logistics: towards eco-efficiency,

122 pages.

PhD thesis, Wageningen University, Wageningen, the Netherlands (2019) With references, with summaries in English and Dutch

ISBN 978-94-6343-914-5

DOI https://doi.org/10.18174/472673 


\section{Contents}

1 Introduction 1

2 Reducing $\mathrm{CO}_{2}$ emissions in temperature-controlled road transportation using the LDVRP model 9

3 The quality-driven vehicle routing problem: Model and application to a case of cooperative logistics

4 Quantifying the environmental and economic benefits of cooperation: A case study in temperature-controlled food logistics

5 Fair gain allocation in eco-efficient vendor-managed inventory cooperation 71

6 Conclusions and general discussion $\quad 87$

$\begin{array}{ll}\text { Appendix } & 97\end{array}$

$\begin{array}{ll}\text { Bibliography } & 103\end{array}$

$\begin{array}{ll}\text { Statement of authorship contribution } & 111\end{array}$

$\begin{array}{ll}\text { Summary } & 113\end{array}$

$\begin{array}{ll}\text { Samenvatting } & 115\end{array}$

$\begin{array}{ll}\text { Acknowledgements } & 118\end{array}$

$\begin{array}{ll}\text { Curriculum Vitae } & 119\end{array}$

$\begin{array}{ll}\text { List of publications } & 120\end{array}$ 


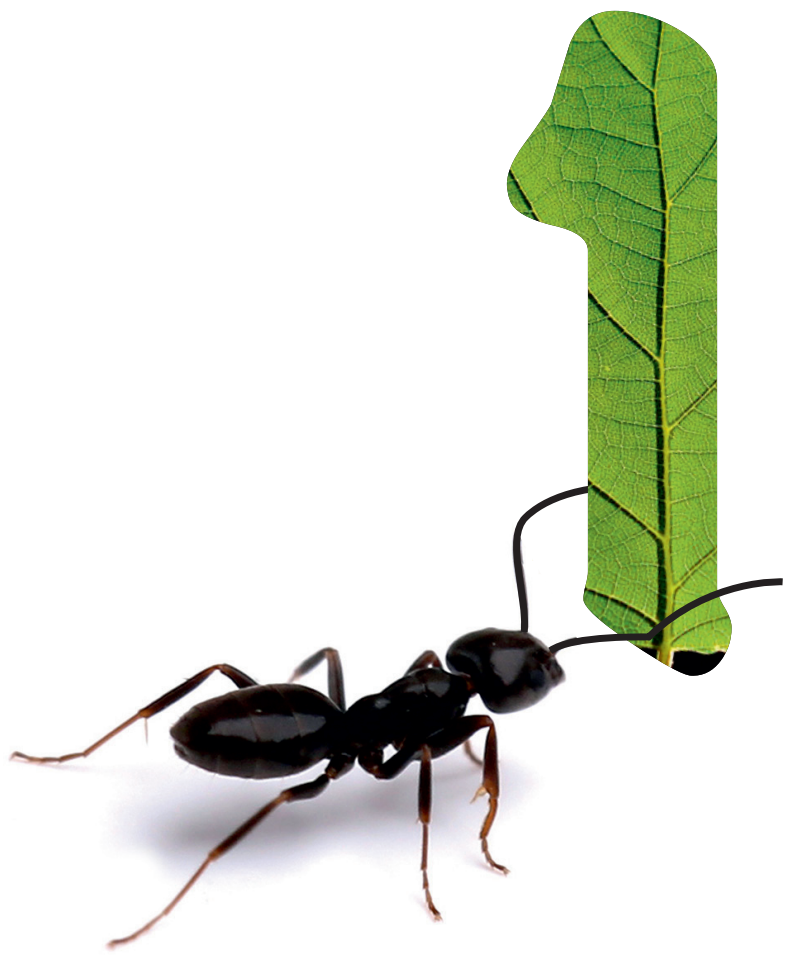




\section{Chapter 1}

\section{Introduction}

Parts of this chapter are based on: H.M. Stellingwerf, A. Kanellopoulos, and J.M. Bloemhof (2019), Sustainable Food Supply Chains: Planning, Design, and Control through Interdisciplinary Methodologies (Eds: Riccardo Accorsi and Riccardo Manzini), Ch. 11: Using vehicle routing models to improve sustainability of temperature-controlled food chains, Elsevier, Amsterdam, The Netherlands.

\subsection{Background}

Food supply chains are challenged to reduce both costs and emissions to improve ecoefficiency and studies have suggested that logistics cooperation can help to achieve this aim. Therefore, the Netherlands Organisation for Scientific Research (NWO) has funded a project called Capitalising on cooperation in sustainable logistics in food chains (CapsLog), which involves Wageningen University, Vrije Universiteit (VU) Amsterdam, Argusl (a supply chain advisory) and a group of Dutch retailers that consider to implement logistics cooperation in order to reduce costs and emissions. This thesis focuses on developing decision support tools that can help designing eco-efficient logistics cooperation in food supply chains.

\section{Food supply chains}

Public awareness about global environmental changes such as air pollution caused by intensified economic activity has increased the need to reduce of environmental impact (Garnett, 2008; Hariga et al., 2017). Road transportation generates significant costs for firms that deliver and collect products but it is also responsible for a large part of global $\mathrm{CO}_{2}$ emissions (Dekker et al., 2012; Palmer, 2007). Food supply chains are more polluting compared to regular supply chains because food is often perishable and temperature control is needed, which requires extra energy, resulting in additional fuel use and emissions (Adekomaya et al., 2016; Tassou et al., 2009). Moreover, food specific properties such as perishability and seasonality make efficient planning of the supply chain more challenging because there is not always a stable supply and shelf life is limited (Van Der Vorst et al., 2009). Food supply chains are challenged to improve economic performance with less environmental impact; they need to become eco-efficient (Banasiket al., 2018). 


\section{Eco-efficiency}

Eco-efficiency was first described by Reijnders (1998) as the reduction of environmental impact of economic activities. In later studies, the definition of eco-efficiency has become more quantitative. For example, in the study of Clavič and Lukman (2007), it is described as the ratio between economic and environmental performance. In this thesis, we adopt a more recent definition of eco-efficient solutions, i.e. solutions for which it is impossible to improve the environmental objective without worsening the economic objective, and vice versa (Neto et al., 2009). Since economic and environmental objectives can be conflicting, multiple alternative eco-efficient solutions may exist (Banasiket al., 2018). However, since our study is focused on food supply chains, food quality is also an important indicator. Food quality can be considered both an economic and an environmental indicator, since quality affects price, but also food waste. Improving costs, emissions and food quality are thus all part of an eco-efficient food supply chain and this thesis will focus on all three indicators when testing eco-efficiency.

\section{Logistics cooperation}

Logistics cooperation has been defined as the situation where two or more autonomous firms work together to plan and execute supply chain operations (Simatupang and Sridharan, 2002). Logistics cooperation between firms of the supply chain has been proposed as a concept that can substantially improve the eco-efficiency of the chain as a whole. This is mainly because firm specific management decisions, like transportation planning and inventory management decisions can be aligned and resources can be used more efficiently (Ramanathan et al., 2014; Vanovermeire et al., 2014; Bloemhofet al., 2015). Establishing effective cooperation between firms is a challenging process. Competition and synergies have to be evaluated and decision support models are required to quantify the potential benefits but also the risks of cooperation in food supply chains. Moreover, the cooperative benefits need to be allocated in a way that is considered fair and acceptable by all partners. Despite the benefits, companies are often hesitant to participate in a cooperation because it might bring advantages to competitors and they find it difficult to agree on gain sharing (Cruijssen et al., 2007a).

\section{Problem definition and research questions}

Food supply chains are challenged to reduce both costs and emissions to improve ecoefficiency. The concept of logistics cooperation is promising but its effects on eco-efficiency in the food supply chain have not been evaluated quantitatively. It is hard to obtain quantitative evidence because of complexity related to food specific aspects such as perishability. Also, there are different forms of cooperation, multiple actors are involved, benefits need to be allocated in a fair way, and economic and environmental objectives can be conflicting. Therefore, decision support models that are able to capture the complexity of establishing effective food logistics cooperation in supply chains need to be developed.

The main question that this thesis aims to answer is: Which decision support models can be used to design eco-efficient logistics cooperation in food supply chains? 


\subsection{Literature and methodological challenges}

\section{Eco-efficiency in food supply chains}

Vehicle and inventory routing problems have been modelled in Operations Research (OR) to optimise logistics decisions in supply chain management. Traditionally, vehicle routing models aim to minimise costs or distance. Recently, green logistics research has also studied how to minimise emissions (Bektaş and Laporte, 2011). However, these green logistics models have not been used to establish a trade-off between cost and emissions (Cheng et al., 2017). As the eco-efficiency objectives can be conflicting, multi-objective models can be used. Those models are designed to comply with multiple objectives simultaneously. Also, they are useful to capture the inherent complexity that characterises temperature-controlled food supply chains (Banasiket al., 2018).

Moreover, to accurately quantify the economic and environmental effects of cooperation in food supply chains, the characteristics of food supply chains need to be considered. Of all foods, $40 \%$ needs refrigeration to guarantee quality. This results in additional energy use, and consequently, fuel and emissions. Moreover, refrigerant leakage causes extra emissions (Adekomaya et al., 2016). Therefore, it is important to consider the effects of temperature control on the costs and emissions.

The first sub question is thus: How to quantify eco-efficiency in temperature-controlled food logistics?

\section{Food quality in cooperative logistics}

In food supply chains, it is important to guarantee food quality. Cooperation is generally expected to reduce costs and emissions but a possible disadvantage is a negative influence on product quality caused by the temperature fluctuations resulting from the increased number of stops on a joint route. Also, transporting multiple products with different optimal temperatures together could negatively affect food quality. In logistics modelling studies, quality decay has been incorporated using different approaches, for example by quantifying remaining shelf life (Amorim and Almada-Lobo, 2014; Soysal et al., 2018), or by using a chance-constrained decay function to mimic quality decay (Ambrosino and Sciomachen, 2007; Osvald and Stirn, 2008; Chen et al., 2009), or by using a temperaturedependent decay function to approximate quality decay (Aung and Chang, 2014; Hsu et al., 2007). However, these studies do not base the decay rate on empirical studies on the temperature-dependency of reactions that cause quality decay. Using a decay function that is related to temperature-dependent reaction speeds in foods could provide more realistic estimations of the reaction rates and consequent quality decay.

The second sub question is thus: How to model temperature-dependent food quality decay in logistics models?

\section{Comparing logistics cooperation concepts}

In literature, different forms of cooperation have been proposed as logistics solutions with promising prospect to improve economic and environmental performance of the 
chain. Different forms of cooperation have been compared qualitatively. Table 1.1 summarises different forms of cooperation and describes their advantages and disadvantages.

Table 1.1: Logistics Cooperation Concepts (LCC), with a description of the cooperation mechanisms, as well as their advantages and disadvantages according to literature.

\begin{tabular}{|c|c|c|c|c|}
\hline LCC & Mechanism & Advantages & Disadvantages & References \\
\hline $\begin{array}{l}\text { Joint } \\
\text { ware- } \\
\text { housing }\end{array}$ & $\begin{array}{l}\text { Opening a } \\
\text { new joint DC } \\
\text { or sharing one }\end{array}$ & $\begin{array}{l}\text { Reduction of inventory } \\
\text { Less pick-ups } \\
\text { Less vehicles } \\
\text { Increased drop size }\end{array}$ & $\begin{array}{l}\text { Investment } \\
\text { New planning } \\
\text { Higher response } \\
\text { time }\end{array}$ & $\begin{array}{l}\text { Aydin and Porteus (2008) } \\
\text { Chopra and Meindl (2007) } \\
\text { Cruijssen et al. (2007a) }\end{array}$ \\
\hline $\begin{array}{l}\text { Vendor- } \\
\text { managed } \\
\text { inventory }\end{array}$ & $\begin{array}{l}\text { Vendor manages } \\
\text { inventory and trans- } \\
\text { port to buyer(s) }\end{array}$ & $\begin{array}{l}\text { Better resource utilisation } \\
\text { Improved inventory } \\
\text { management } \\
\text { Higher service levels } \\
\text { Reduced bullwhip }\end{array}$ & $\begin{array}{l}\text { Requires } \\
\text { advanced } \\
\text { IT facilities }\end{array}$ & $\begin{array}{l}\text { Disney and Towill (2003) } \\
\text { Coelho et al. (2013) } \\
\text { Bazan et al. (2015) }\end{array}$ \\
\hline $\begin{array}{l}\text { Transport } \\
\text { bundling }\end{array}$ & $\begin{array}{l}\text { Node exchange } \\
\text { to enable combining } \\
\text { vehicle loads }\end{array}$ & $\begin{array}{l}\text { More vehicle load } \\
\text { Higher delivery frequency } \\
\text { Higher service levels }\end{array}$ & $\begin{array}{l}\text { Increased opera- } \\
\text { tional time and } \\
\text { handling costs }\end{array}$ & Kreutzberger (2010) \\
\hline $\begin{array}{l}\text { Joint route } \\
\text { planning }\end{array}$ & $\begin{array}{l}\text { Jointly optimising } \\
\text { daily routing } \\
\text { decisions }\end{array}$ & $\begin{array}{l}\text { More reliable } \\
\text { Less slack time } \\
\text { Less vehicles } \\
\text { Better vehicle utilisation }\end{array}$ & $\begin{array}{l}\text { Increased schedu- } \\
\text { ling complexity } \\
\text { Necessity stand- } \\
\text { by vehicles }\end{array}$ & $\begin{array}{l}\text { Cruijssen et al. (2007b) } \\
\text { Conzalez-Feliu et al. (2010) }\end{array}$ \\
\hline
\end{tabular}

A qualitative comparison of different forms of logistics cooperation can provide insight, but without a quantitative comparison, it is hard for partners to choose a form of cooperation. Also, quantification of benefits can help to convince partners to cooperate. However, in quantitative studies, authors often compare cooperation to a non-cooperative scenario. Multiple forms of cooperation are possible, and should be compared to each other based on different multiple indicators, such as costs and emissions.

In order to quantify the eco-efficiency of a cooperative logistics systems, multiple indicators should be considered. For example, costs, emissions and food quality. Nonetheless, minimising costs does not always yield the same solution as minimising emissions and trade-offs exist. For partners to be able to make a decision on a cooperative transport plan, it is thus important to clarify if there are trade-offs between the different objectives, and to quantify these trade-offs.

The third sub question is thus: What are the effects of different forms of cooperation on ecoefficiency?

\section{Gain allocation for eco-efficient food supply chain cooperation}

Cost savings are an important reason for partners to cooperate. However, when a form of cooperation is chosen, and partners agree on a cooperative logistics plan, they also need to decide how to divide the resulting benefits in a fair way. Agreeing on a fair gain division is generally hard for partners and research shows that this is one of the most common impediments to cooperation (Cruijssen et al., 2007c). It is thus important to find a gain allocation that is considered fair by all participants. 
Gain allocation methods have been used to identify potential coalitions and to fairly allocate the benefits of the coalitions to all cooperating partners (Nagarajan and Sošić, 2008). Tijs and Driessen (1986) have summarised different gain allocation methods. In most gain allocation methods, the benefits or cost allocated to a partner are related to the partner's contribution to the group's cost savings (Guajardo and Rönnqvist, 2016). Recent case studies that compare different cost allocation methods are, for example, Frisk et al. (2010), Vanovermeire et al. (2014), and Wang et al. (2017). Some studies (Frisket al., 2010; Vanovermeire et al., 2014; Jonkman et al., 2019) conclude that cooperation can also result in environmental benefits. However, in these studies the contribution of the partners to $\mathrm{CO}_{2}$ emissions saving is not taken into account in the allocation of the cooperative benefits. To stimulate eco-efficient forms of cooperation, partners should not only be rewarded for reducing cooperative costs, but also for improving performance of other indicators such as emissions.

The fourth sub question is thus: How can gain allocation be applied such that eco-efficient forms of logistics cooperation are stimulated?

\subsection{Outline of thesis}

The problems and the concepts addressed in the research questions, are linked to each other and this can be visualised as shown in Figure 1.1, which summarises the modelling framework used in this thesis. It shows that we aim to build models that can be used to evaluate logistics cooperation concepts based on their eco-efficiency, while considering supply chain characteristics, and that we aim to allocate gains based on the contribution of the cooperative partners to eco-efficiency.

In order to study how cooperation can be used to improve eco-efficiency, we need methods to quantify the eco-efficiency of food supply chains. Therefore, in Chapter 2, a green vehicle routing problem is extended to account for the costs and emissions caused by temperature control. Cooperation in food supply chains can result in significant savings in costs and emissions. However, cooperative routes can result in more door openings, and temperature fluctuations, which could have a negative effect on the quality of the temperature sensitive products delivered. Therefore, in Chapter 3 we extend the model of Chapter 2 such that it can be used to quantify the effects of door openings and temperature fluctuations on the quality of the food transported. Also, we study the effects of transporting different products with different optimal temperatures (i.e. not all products are transported at their optimal temperature) on the quality of the products. 


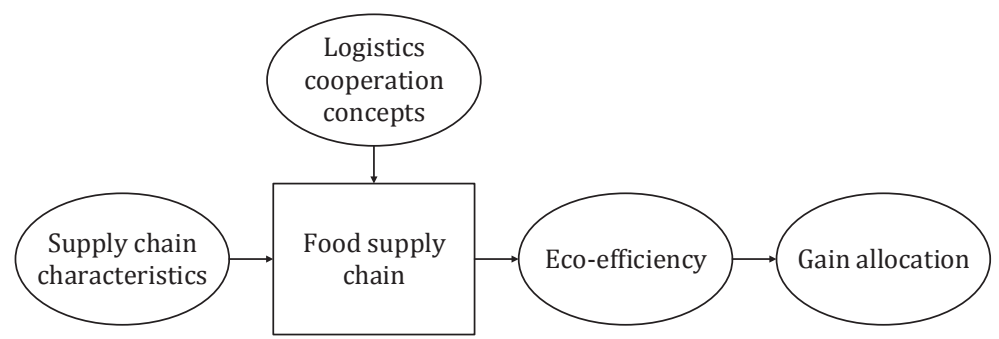

Figure 1.1: Modelling framework used in the thesis.

In Chapter 4, we quantify and compare the economic and environmental benefits of different forms of cooperation (JRP and vendor-managed inventory, VMI) with a non-cooperative scenario in a case study in frozen food logistics. We study eco-efficiency of VMI cooperation, as well as the trade-off between emissions and product age. One of the impediments to cooperation is how to share the resulting gains. In Chapter 5, we propose a methodology to turn this impediment into an opportunity to stimulate eco-efficient forms of cooperation. Gain allocation is generally based on each partner's contribution to saving costs, and we show how it can be used to base the allocation on each partner's contribution to costs as well as to emissions. Building on the insights from the previous chapters, Chapter 6 is used to draw general conclusions, discuss implications and limitations of this work, put it in a broader perspective, and suggest future research directions. 
1.3. Outline of thesis 


$$
2
$$




\title{
Chapter 2
}

\section{Reducing $\mathrm{CO}_{2}$ emissions in temperature-controlled road transportation using the LDVRP model}

\begin{abstract}
Temperature-controlled transport is needed to maintain the quality of products such as fresh and frozen foods and pharmaceuticals. Road transportation is responsible for a considerable part of global emissions. Temperature-controlled transportation exhausts even more emissions than ambient temperature transport because of the extra fuel requirements for cooling and because of leakage of refrigerant. The transportation sector is under pressure to improve both its environmental and economic performance. To explore opportunities to reach this goal, the Load-Dependent Vehicle Routing Problem (LDVRP) model has been developed to optimise routing decisions taking into account fuel consumption and emissions related to the load of the vehicle. However, this model does not take refrigeration related emissions into account. We therefore propose an extension of the LDVRP model to optimise routing decisions and to account for refrigeration emissions in temperature-controlled transportation systems. This extended LDVRP model is applied in a case study in the Dutch frozen food industry. We show that taking the emissions caused by refrigeration in road transportation can result in different optimal routes and speeds compared with the LDVRP model and the standard Vehicle Routing Problem model. Moreover, taking the emissions caused by refrigeration into account improves the estimation of emissions related to temperature-controlled transportation. This model can help to reduce emissions of temperature-controlled road transportation.
\end{abstract}

This chapter is based on: Stellingwerf, H.M., Kanellopoulos, A., van der Vorst, J.G.A.J., Bloemhof, J.M. (2018). Reducing $\mathrm{CO}_{2}$ emissions in temperature-controlled road transportation using the LDVRP model. Transportation Research part D: Transport and Environment, 65, 178-193, 2018 


\subsection{Introduction}

Transportation of goods results in substantial economic and environmental consequences (Palmer , 2007). The percentage of $\mathrm{CO}_{2}$ emissions caused by vehicle transportation in the European Union has increased from 5.6\% in 1990 to 9\% in 2014; worldwide, transportation causes $14 \%$ of the global $\mathrm{CO}_{2}$ emissions Dekker et al. (2012). Greenhouse gas emissions from conventional diesel engine vapour compression refrigeration systems can be as high as $40 \%$ of the vehicle's emissions Tassou et al. (2009). In general, current transportation systems are far from efficient and the problem is more severe in temperaturecontrolled transportation systems of, for example, frozen food and pharmaceuticals, for which additional energy is needed to regulate temperature and ensure quality, product safety and shelf-life (Adekomaya et al., 2016; Ketzenberg et al., 2015). It is therefore important to keep the temperature at the appropriate level. A survey showed that the thermal energy requirement is around $15 \%$ to $25 \%$ of the motive energy requirement of vehicles (Tassou et al., 2009) and therefore temperature-controlled transportation is more polluting than ambient transportation.

To improve the efficiency of current temperature-controlled transportation systems, we need appropriate decision support tools to compare different options to eliminate inefficiencies. Models based on the Vehicle Routing Problem (VRP) have been proposed to optimise operational routing decisions for transportation systems of various commodities. The basic variants of these models minimise transportation costs or transportation distance and provide an optimal route to deliver or collect commodities (Toth and Vigo, 2002). Some variants of VRP models, known as Creen Vehicle Routing Problem (CVRP) models (Lin et al., 2014) have also been used to minimise environmental impact, most often expressed as carbon dioxide $\left(\mathrm{CO}_{2}\right)$ emissions. Demir et al. (2014) summarise different approaches to the GVRP. The Load-Dependent VRP (LDVRP) model can be considered as a special case of a GVRP model that takes the load and the order of unloading into account when calculating fuel consumption (Bektaş and Laporte, 2011; Zachariadis et al., 2015). Existing green logistics VRP models do not account for the substantial emissions exhausted as a result of temperature control. Accurate quantification of the emissions of temperature controlled transportation requires not only to consider emissions caused by fuel use needed for driving, but also emissions caused by fuel use for refrigeration and refrigerant leakage. Consequently, existing green logistics VRP models need to be extended before they can be used to optimise operational decisions for temperature-controlled transportation systems.

The objective of this Chapter is to propose an extension of the LDVRP model to minimise emissions in temperature-controlled transportation systems. We propose a metric of $\mathrm{CO}_{2}$ emissions that, next to emissions caused by fuel use for moving the vehicle, also considers emissions caused by fuel use for cooling the vehicle as well as emissions caused by leakage of refrigerant. To that aim, a Load-Dependent VRP is extended to account for emissions caused by fuel used for temperature control and for refrigerant leakage. This model is applied to a case study in frozen food transportation in the Netherlands. In Section 2.2, we present the methodology, in which we describe an LDVRP model and the ex- 
tensions required to take emissions caused by refrigeration into account. The case study and the calculations are presented in Section 2.3 and the results in Section 2.4. We conclude with the discussion and conclusions in Section 2.5 .

\subsection{Methodology}

This section first gives a review of relevant LDVRP literature and describes the LDVRP model. Then, characteristics of temperature controlled transportation are described these characteristics are translated into a LDVRP extension. In the appendix, all nomenclature used is summarised (Table A.1), as well as the decision variables (Table A.2) the values used to run the model (Table A.3) and all parameters are defined there as well (Table A.4).

\section{The green vehicle routing problem: review of relevant literature}

VRP models are used to find optimal routes for delivering or collecting products, mostly by minimising total distance (Toth and Vigo, 2002). However, different objective functions have been used. For example, to minimise environmental impacts caused by the distance travelled, green VRPs have been developed (Demiret al., 2014; Lin et al., 2014; Jaehn, 2016). In ambient temperature transport, $\mathrm{CO}_{2}$ emissions are linearly related to fuel consumption, which in turn is linearly related to the loaded distance (i.e. weight multiplied by distance) travelled. Because the fuel consumption and thus the emissions are directly related to the weighted distance, the order of unloading can have a significant impact on the pollution caused by a route (Kara et al., 2007; Xiao et al., 2012; Molina et al., 2014; Zachariadis et al., 2015). The recently proposed LDVRP model considers the load and the order of unloading when comparing routes, for example, by minimising total $\mathrm{CO}_{2}$ emissions and transportation costs (Kara et al., 2007; Bektaş and Laporte, 2011; Xiao et al., 2012; Bing et al., 2014; Zachariadis et al., 2015).

Kara et al. (2007) use the LDVRP to minimise energy use on a route, and they provide two examples to show that the LDVRP model gives different results than a distance minimising model. Xiao et al. (2012) consider the load into account in a fuel consumption optimisation model. Like Kara et al. (2007), they show that their model gives a different result than distance minimisation. Also Ahn and Rakha (2008) describe show that taking the load into account can change the best route from an environmental and an energy perspective: the fastest route is no longer the best.

Zachariadis et al. (2015) use the LDVRP model proposed by Kara et al. (2007) and extend it to account for pickup and delivery time in order to analyse the influence of the maximum cargo to empty weight ratio. The authors state that the LDVRP model is suitable for optimising transportation operations when the weight of the transported cargo has a significant contribution to the gross vehicle weight, such as logistics operations for supermarkets. In this case, the LDVRP model generates a more sensible transportation plan compared with basic VRP models (Zachariadis et al., 2015). For a sensitivity analysis, the authors compare the objective function values of two different routes with loads of varying weight. 
Most LDVRP studies use one objective function. For example, Kara et al. (2007) and Xiao et al. (2012) both add the weighted distance (which they translate to energy and fuel consumption, respectively) into a cost function. Molina et al. (2014) combine three different objectives (i.e. to minimise internal costs, $\mathrm{CO}_{2}$ emissions, and NOx emissions) into one function. The Pollution Routing Problem is an example of an LDVRP that takes both the economic and environmental impact of different routes into account (Bektaş and Laporte, 2011). That study takes a broader view than the standard VRP by analysing routes based on four indicators: costs, emissions, distance, and time.

\section{The Load Dependent Vehicle Routing Problem}

An LDVRP is formulated based on the studies described above. Bektaş and Laporte (2011) used the LDVRP model to minimise environmental impacts, such as energy use. The model was adjusted to account for multiple vehicles. The total energy use in ambient temperature transportation systems is the motive energy. The motive energy requirement depends on the distance driven, the weight transported over that distance, the steepness of the road $(\theta)$ and the air density $(\rho)$. The first objective function (Equation 2.1) minimises motive energy and is based on that study:

$$
\text { Minimise }\left\{P_{m}=\sum_{i \in V} \sum_{j \in V} \sum_{k \in K} \alpha_{i j} w_{i j}^{k} c_{i j}+\sum_{i \in V} \sum_{j \in V} \sum_{k \in K} \beta^{k} x_{i j}^{k} v_{i j}^{2} c_{i j}\right\},
$$

where $P_{m}$ is the total motive energy requirement of a route ( $\left.\mathrm{kWh}\right), x_{i j}^{k}$ is a binary variable that equals 1 if and only if the route between node $i$ and node $j$ is taken with vehicle $k$, $y_{i j}^{k}$ is the weight of vehicle $k$, including the load that is transported from node $i$ to node $j, c_{i j}$ is the distance between node $i$ and node $j(\mathrm{~m})$ and $v_{i j}$ is the speed at which the distance between $i$ and $j$ is traversed $(\mathrm{m} / \mathrm{s})$, and $\alpha_{i j}$ is the arc-specific constant, and $\beta$ is the vehicle-specific constant. Equations (2.2) and (2.3) show how these constants are calculated:

$$
\alpha_{i j}=a+g \sin \theta_{i j}+g C_{r} \cos \theta_{i j},
$$

where $a$ is the acceleration of the vehicle $\left(\mathrm{m} / \mathrm{s}^{2}\right), g$ is the gravitation constant $\left(\mathrm{m} / \mathrm{s}^{2}\right), \theta_{i j}$ refers to the average slope on $\operatorname{arc} i j\left({ }^{\circ}\right), C_{r}$ is the rolling resistance (dimensionless). The vehicle-specific constant is calculated as

$$
\beta=0.5 C_{d} A \rho,
$$

where $C_{d}$ is the drag coefficient (dimensionless), $A$ is the frontal area of the vehicle $\left(\mathrm{m}^{2}\right)$, and $\rho$ is the air density $\left(\mathrm{kg} / \mathrm{m}^{3}\right)$.

In (2.4) fuel use for ambient (motive) transport $\left(f_{m}\right)$ is calculated by summing up the power requirements for all routes and converting those into fuel use. This is achieved by dividing the power by $3.6 \times 10^{6}$ to convert Joule (J) to kilowatt-hour ( $\mathrm{kWh}$ ), by the chemical to motive energy conversion efficiency $\left(\eta_{m}\right)$, and by the energy content of the fuel $\left(P_{f}\right)$ :

$$
f_{m}=\frac{\sum_{i \in V} \sum_{j \in V} P_{i j}}{3.6 \times 10^{6} P_{f} \eta_{m}} .
$$


The emissions from ambient (motive) transport are linearly related to fuel use:

$$
E_{m}=f_{m} e_{f}
$$

where $e_{f}$ is the emissions factor which converts fuel use into $\mathrm{CO}_{2}$ emissions $(\mathrm{kg} / \mathrm{L})$, and $E_{m}$ are the $\mathrm{CO}_{2}$ emissions of ambient transport (kg). Equation 2.5 shows that motive energy and motive emissions are linearly related. This means that in case of ambient transport (no thermal energy), minimising motive energy and minimising emissions will give the same results (Palmer, 2007).

To also analyse the economic consequences of using different VRP-based model, an operational cost function is constructed (Equation 2.6). From an operational perspective, fuel costs and wage costs are the most important costs. Fuel costs depend on energy use and wage costs depend on the time that a driver spends on a route.

The total cost can be calculated as follows by adding wage cost and fuel cost:

$$
C=\sum_{i \in V} \sum_{j \in V} \sum_{k \in K} \sum_{t \in T} \frac{c_{w} c_{i j} x_{i j}^{k t}}{v_{i j}}+\sum_{i \in V^{\prime}} \sum_{j \in V} \sum_{k \in K} \sum_{t \in T} c_{w} x_{i j}^{k t} s+\left(f_{a}+f_{r}\right) c_{f},
$$

where $C$ refers to the total cost $(€), c_{w}$ is the driver wage per time unit $(€ / s), c_{f}$ is the unit fuel cost $(€ / \mathrm{L})$, and $c_{w}$ is the unit wage cost $(€ / \mathrm{s})$.

We formulate the LDVRP constraints based on (Kara et al., 2007) and extend them such that they fit the requirements of transportation with multiple vehicles. Constraints (2.7) - (2.14) were adjusted to account for multiple vehicles and constraint (2.15) was added to limit the maximum driving time per vehicle, such that the proposed solutions are in line with the regulations for driver's working times (Molina et al., 2014). Because of the explicit vehicle numbering, explicit sub-tour elimination constraints were needed, so constraints (2.16) and (2.17) were added (Miller et al., 1960). Constraint (2.18) ensures that no vehicles drive to locations without demand. As a result of accounting for multiple vehicles, it was necessary to add constraints (2.19) - (2.21). Explanation of all constraints is given after their formulation. 
subject to

$$
\begin{aligned}
& \sum_{k \in K} \sum_{i \in V^{\prime}} x_{0 i}^{k} \leq K \\
& \sum_{k \in K} \sum_{i \in V} x_{i j}=1 \quad q_{j}>0, j \in V^{\prime}, \\
& \sum_{k \in K \mid q_{i}>0,} \sum_{j \in V} x_{i j}=1 \quad i \in V^{\prime}, \\
& \sum_{i \in V} w_{j i}^{k}-\sum_{i \in V \mid i \neq j} w_{i j}^{k}=q_{i}^{k} \quad j \in V^{\prime}, k \in K, \\
& w_{i 0}^{k}=L_{0}^{k} x_{i 0}^{k} \quad i \in V^{\prime}, k \in K, \\
& w_{i 0}^{k} \leq\left(L^{k}+L_{0}^{k}-q_{i}\right) x_{i j}^{k} i \in V, j \in V, k \in K \text {, } \\
& w_{i j}^{k} \geq\left(L_{0}^{k}+q_{j}\right) x_{i j}^{k} \quad i \in V, j \in V, k \in K, \\
& x_{i j}^{k} \in\{0,1\} \quad i \in V, j \in V, k \in K, \\
& \sum_{i \in V} \sum_{j \in V^{\prime}} \frac{c_{i j} x_{i j}^{k}}{v_{i j}}+\sum_{i \in V} \sum_{j \in V^{\prime}} x_{i j}^{k} s_{i} \leq d \\
& k \in K \\
& u_{i}-u_{j}+L^{k} x_{i j}^{k} \leq L_{k}-q_{j} \quad i \in V^{\prime}, j \in V, k \in K \\
& q_{i} \leq u_{i} \leq L^{k} \quad i \in V^{\prime}, k \in K \\
& \sum_{j \in V} x_{i j}^{k}=0 \quad q_{i}=0, i \in V^{\prime}, k \in K, \\
& \sum_{i \in V} \sum_{j \in V} \sum_{k \in K} x_{i j}^{k}=0 \quad i=j \\
& \sum_{i \in V^{\prime}} \sum_{k \in K} x_{i 0}^{k}=\sum_{i \in V^{\prime}} \sum_{k \in K} x_{0 i}^{k} \\
& \sum_{j \in V} x_{0 j}^{k} \leq 1 \quad k \in K
\end{aligned}
$$

Constraint (2.7) ensures that no more than the maximum number of vehicles available $(K)$ leave the CDC. Constraints (2.8) ensure that each node with demand $\left(q_{i}\right)$ is visited once and constraints (2.9) cause each node with demand to also be left once. Constraints (2.10) are balance constraints; after a node has been visited, the load of the vehicle diminishes with the demand of the node just visited. Constraints (2.10) force the vehicles to return to the depot empty ( $L_{0}^{k}$ is the tare, i.e. the empty weight of the vehicle). Constraints (2.12) and (2.13) put boundaries on the minimum and maximum weight ( $L^{k}$ is 
the total weight of vehicle $k$ ) that can be transported over an edge, and connect the decision variables $x_{i j}^{k}$ and $y_{i j}^{k}$ such that the objective functions remain linear. Constraints (2.14) forces $x_{i j}^{k}$ to be integer; a path is either taken or it is not. Constraints (2.15) limit the maximum working time per driver $(d) ; s$ is the time needed for unloading. Constraints (2.16) and (2.17) are Miller-Tucker-Zemlin sub-tour elimination constraints (Miller et al., 1960). Constraints (2.18) ensure that there are no routes to locations without demand. Constraints (2.19) do not allow the model to suggest routes between the same location. Constraint (2.20) states that the number of vehicles leaving the depot should equal the number of vehicles returning. Constraints (2.21) force the model to use a new vehicle for a new route from the depot.

\section{Temperature controlled road transportation: a review of relevant literature}

Food transport refrigeration causes additional emissions compared to ambient transport (Adekomaya et al., 2016). Most refrigeration systems for food transportation use diesel driven vapour compression (Tassou et al., 2009). The chemical energy from the diesel is converted to electrical energy and the constant $\eta_{e}$ is used to describe the efficiency of this conversion. Then the electrical energy is used to drive the transport of heat from the inside to the outside of the vehicle. This heat transport can be described with the Coefficient of Performance (COP). The COP describes the ratio of heat removed as function of energy supplied, is generally between 0.5 and 1.5 for refrigerated transport of chilled food (at $2^{\circ} \mathrm{C}$ ) (Tassou et al., 2009). The COP can be above 1 because the electric power is used to transport energy (heat) instead of converting it. In frozen food, where the COP is generally below 1 , there are thus two conversions (from chemical to electrical to thermal) in which energy is lost, which increases the energy demand for cooling. In the field of thermal engineering, some studies use thermodynamics to estimate how much heat (energy) should be removed in order to keep the temperature in the vehicle stable (Kondjoyan, 2006; Pham, 2006). These studies however require detailed knowledge of the values of many technical parameters, including the dimensions and the direction of the airflow, the composition of the air and the composition of the products. In daily route planning practice, these data are often unavailable. In this Chapter, we propose a more operational approach to the amount of energy that needs to be removed from the vehicle for a stable temperature. Thermal energy is needed to compensate for the heat that enters through the vehicle wall during the trip (Tassou et al., 2009; James and James, 2010), and for the heat that enters through the door when the vehicle is opened (Tso et al., 2002). Tassou et al. (2009) described the heat entering the vehicle wall as a function of conductivity, the difference between the temperature inside and outside the vehicle wall, and the surface area. Tso et al. (2002) measured how much the temperature of the vehicle air increases when the door opens.

The refrigeration system produces emissions because of fuel usage but it also does so by leaking refrigerant (Adekomaya et al., 2016). The vapour compression system used for refrigeration of the vehicle load slowly leaks refrigerant to the environment, at a rate of between 10 to $37 \%$ of the refrigerant charge peryear (Spence et al., 2004). This refrigerant leakage can cause the emissions that are caused by using fuel for temperature control to increase with 21\% (Koehler et al., 1997). 
An overview of types of temperature controlled vehicles, cooling technologies, and future improvements for cooling technologies can be found in Tassou et al. (2009). Adekomaya et al. (2016) also focus on different technologies to improve energy consumption, and they discuss worldwide energy use for cooled transport. However, these papers do not study the impact of routing decisions on refrigeration emissions. If temperature control is taken into account in routing decisions, it could already help to reduce temperature control emissions. Moreover, when a company considers using a new technology for cooling, a Temperature Controlled LDVRP could help to estimate the effects on costs, fuel and emissions from an operational perspective. Therefore, we propose an extension of the LDVRP model to account for both emissions caused by motive energy requirements and emissions caused by refrigeration energy requirements and leakage of refrigerant.

\section{The Temperature Controlled LDVRP model}

The standard LDVRP model does not account explicitly emissions caused by refrigeration systems in road transportation. For that reason, it underestimates the environmental impact of temperature-controlled transportation systems. Minimising carbon dioxide emissions is a way to minimise environmental impact. The objective function (2.22) describes total emissions from refrigerated road transportation.

$$
\text { Minimise }\left\{E=E_{m}+E_{t}\right\} \text {, }
$$

where $E$ represents the emissions $\left(\mathrm{kg} \mathrm{CO}_{2}\right), E_{t}$ represents the emissions because of thermal energy and refrigerant leakage and $E_{m}$ represents the motive emissions, which are the total emissions in ambient transport. $E_{m}$ is given in Equation (2.5).

Together with constraints (2.7) - (2.21), objective (2.22) is an extended version of the LDVRP model for temperature-controlled road transportation, which is why we call it the temperature-controlled LDVRP (TCLDVRP). Thermal emissions and the components that make up those emissions are given in Equations (2.23) - (2.28).

$$
E_{t}=F_{t} \times e_{f} \times e_{r},
$$

where $F_{t}$ represents fuel use for thermal energy $(\mathrm{L}), e_{f}$ is the conversion factor from fuel to emissions caused by fuel, and $e_{r}$ is a factor that converts emissions caused by thermal energy use to emissions caused by thermal energy use and refrigerant leakage.

$$
F_{t}=\frac{Q_{c}}{\eta_{e} \times C O P \times E_{d}}
$$

where $Q_{c}$ is the thermal energy (i.e. heat removed from the inside of the vehicle, in $\mathrm{kWh}$ ), $\eta_{e}$ is the conversion efficiency from chemical energy of the diesel to electrical energy that drives the cooling engine; COP is the Coefficient of Performance, which is a measure for 
how much thermal energy can be removed with the electrical energy supplied, and $E_{d}$ is the energy content of the diesel (in kWh/L). The COP is given in Equation (2.25).

$$
C O P=\frac{Q_{c}}{W_{g}}
$$

where $Q_{c}$ is the net heat absorbed at the cold side of the cooling device, and $W_{g}$ is the electrical power applied to do so (Tassou et al., 2009).

Combining the work of (Tso et al., 2002) and (Tassou et al., 2009), we describe the thermal energy requirement $\left(Q_{c}\right)$ as sum of the heat entering through the vehicle wall $(H W)$ and the energy from the heat coming in when the door is opened at a stop $(H S)$ in Equation (2.26).

$$
Q_{c}=H W+H S
$$

$H W$ and $H S$ are calculated in Equations (2.27) and (2.28), respectively.

$$
H W=\sum_{i \in V} \sum_{j \in V} \sum_{k \in K} U^{k} S^{k} \Delta T_{i j} \frac{c_{i j} x_{i j}}{v_{i j}}
$$

where $U^{k}$ is the heat transfer coefficient of vehicle $k(\mathrm{~W} / \mathrm{m} 2 / \mathrm{K}), S^{k}$ is the mean section of the vehicle body in $\mathrm{m}^{2}$ (which is the square root of the product of the inside and the outside area of the vehicle), $\Delta T_{i j}$ is the average air temperature difference between the inside and outside of the body on $\operatorname{arc} i j(\mathrm{~K})$, and with $\frac{c_{i j} x_{i j}^{m}}{v_{i j}}$, the arc crossing time is calculated.

$$
H S=\sum_{i \in V^{\prime}} \sum_{j \in V^{\prime}} \sum_{k \in K} x_{i j}^{k} h_{i}
$$

where $h_{i}$ is the amount of heat entering the vehicle when the door is opened during a stop at location $\mathrm{i}(\mathrm{kWh})$ and summing over $x_{i} j^{k}$ results in the number of stops when the depot is excluded.

\subsection{Case study}

This case study focuses on frozen food road transportation in the Netherlands. More specifically, we focus on optimising the daily routing decisions of a large supermarket consortium that is responsible for $30 \%$ of the market share of supermarkets, i.e. nine supermarket chains in the Netherlands. These supermarket chains order their products together. The products then have to be transported from the central distribution centre (CDC) to the distribution centres (DCs) of the different supermarket chains. Some DCs want a delivery almost every day; others prefer less frequent deliveries. On a daily basis, 
a routing plan is made according to the demand. Currently, the $C D C$ tries to minimise the total distance, but it is also interested in minimising total fuel consumption.

\section{Data and assumptions}

\section{Case study data}

In this case study, the analysis is based on the orders of the main distribution centres (DCs, numbered 1 to 9) of the nine supermarket chains in October 2014, which are delivered from the CDC (numbered 0). On each day except Sundays, the CDC sends frozen products to the DCs in temperature-controlled vehicles. Note that not every DC has demand on every day and demand varies per day for each retailer. DCs without demand on a certain day are not visited on that day.

\section{Distances}

The distances between the locations are estimated based the road distances between the postal codes of the locations using the ESRI ArcGIS software. The distances between the nodes are shown in Table 2.1.

Table 2.1: Distances (in $\mathrm{km}$ ) between the depot (0) and the DCs (1-9).

\begin{tabular}{rrrrrrrrrrr}
\hline & 0 & 1 & 2 & 3 & 4 & 5 & 6 & 7 & 8 & 9 \\
\hline 0 & 0 & 91 & 6 & 134 & 82 & 117 & 74 & 192 & 129 & 120 \\
1 & 91 & 0 & 91 & 75 & 134 & 43 & 20 & 155 & 59 & 122 \\
2 & 6 & 91 & 0 & 134 & 84 & 117 & 75 & 194 & 129 & 123 \\
3 & 134 & 75 & 134 & 0 & 168 & 46 & 94 & 90 & 29 & 118 \\
4 & 82 & 134 & 84 & 168 & 0 & 158 & 140 & 180 & 169 & 90 \\
5 & 117 & 43 & 117 & 46 & 158 & 0 & 59 & 117 & 18 & 128 \\
6 & 74 & 20 & 75 & 94 & 140 & 59 & 0 & 174 & 77 & 128 \\
7 & 192 & 155 & 194 & 90 & 180 & 117 & 174 & 0 & 99 & 110 \\
8 & 129 & 59 & 129 & 29 & 169 & 18 & 77 & 99 & 0 & 137 \\
9 & 120 & 122 & 123 & 118 & 90 & 128 & 128 & 110 & 137 & 0 \\
\hline
\end{tabular}

\section{Driving speed}

To estimate the average speed between nodes, we took the traffic situation in the Netherlands into account. In general, the west of the Netherlands is more densely populated than the east. Therefore, travelling to the west is generally slower than travelling to the east. This is especially the case in the morning, when most of the traffic jams occur. Moreover, most of the transportation in this case study occurs in the morning. To generate the speed matrix in Table 2.2, we assumed that the speed of arcs going eastwards follow a normal distribution with a mean of $62.5 \mathrm{~km} / \mathrm{h}$ and a standard deviation of $3 \mathrm{~km} / \mathrm{h}$, while the speed of arcs going westwards follow a normal distribution with a mean of $47.5 \mathrm{~km} / \mathrm{h}$ and a standard deviation of $3 \mathrm{~km} / \mathrm{h}$ (cf. Bektaş and Laporte (2011)).

\section{Slope}

Between the west and the east of the Netherlands, there is a very small incline of $0.01^{\circ}$ (assuming an altitude difference of $52 \mathrm{~m}$ at a horizontal displacement of $300 \mathrm{~km}$ ). With Equation 2.2, we use this slope to calculate $\alpha_{i j}$ for the arcs going to the west (0.0964) and 
Table 2.2: Speeds (in $\mathrm{km} / \mathrm{h}$ ) between the depot (0) and the DCs (1-9).

\begin{tabular}{rrrrrrrrrrr}
\hline & 0 & 1 & 2 & 3 & 4 & 5 & 6 & 7 & 8 & 9 \\
\hline 0 & & 45.9 & 50.7 & 51.4 & 63.4 & 65.9 & 50.5 & 46.7 & 65 & 52.9 \\
1 & 59.1 & & 42.9 & 48.1 & 60.2 & 65.3 & 44.5 & 46.9 & 66.9 & 41.9 \\
2 & 64.2 & 60.3 & & 60.2 & 66.4 & 63.6 & 59.1 & 50.5 & 57.2 & 60.1 \\
3 & 61 & 64.7 & 44.2 & & 67.9 & 58.9 & 51.3 & 52.3 & 61 & 44.5 \\
4 & 41.8 & 49.7 & 50 & 50.4 & & 65.5 & 48.2 & 48.1 & 63.2 & 46.8 \\
5 & 50.7 & 52.1 & 46.8 & 47.8 & 42.3 & & 42.5 & 50.2 & 50 & 44.7 \\
6 & 62.5 & 63.1 & 44.9 & 65.3 & 65.2 & 61.1 & & 50.7 & 62 & 64.3 \\
7 & 62.8 & 56.8 & 59.7 & 59.8 & 59.3 & 59.2 & 60.9 & & 60.6 & 54.9 \\
8 & 43.1 & 51.3 & 45.3 & 40.1 & 44 & 62 & 50.9 & 51.3 & & 46.3 \\
9 & 62.7 & 57 & 48.7 & 57.5 & 61.7 & 62 & 50.5 & 48 & 57.3 & \\
\hline
\end{tabular}

$\alpha_{i j}$ for the arcs going to the east (0.0998).

\section{Vehicle}

The LDVRP described in Section 2.2 was adjusted to account for multiple vehicles. However, our goal was to use this extension to be able to set a limit to the maximum driving time per vehicle (driver) per day. We do not assume a heterogeneous fleet as the majority of refrigerated food road transport is conducted with semi-trailer with insulated rigid boxes. Therefore we assume this type of vehicles. The internal and external dimensions of the vehicle are assumed to be $(\mid \times w \times h$, in meters): $13.56 \times 2.6 \times 2.75$ and $13.35 \times 2.46 \times 2.5$, respectively (Tassou et al., 2009). The empty weight of each of the vehicles is $10,000 \mathrm{~kg}$ and the maximum load is $30,000 \mathrm{~kg}$. Equation (2.3) is used to calculate of the vehicle-specific constant $\beta^{m}$, we assume the coefficient of $\operatorname{drag}\left(C_{d}\right)$ is 0.7 , the air density $(\rho)$ is $1.2041 \mathrm{~kg} / \mathrm{m}^{3}$ (Bektaş and Laporte, 2011), and the frontal area of the vehicle is $7.15 \mathrm{~m}^{2}$, which results in a $\beta$ value of 3.013 . We assume the heat transfer coefficient (U) of the vehicle wall to be $0.7 \mathrm{~W} / \mathrm{m}^{2} / \mathrm{K}$ (Tassou et al., 2009).

\section{Temperature}

We assume the ambient temperature is $30^{\circ} \mathrm{C}$ and the temperature inside the vehicle when transporting frozen goods is $-18^{\circ} \mathrm{C}$ (Tassou et al., 2009). When the door opens, we assume the air temperature increases by $8^{\circ} \mathrm{C}$ (Tso et al., 2002; Estrada-Flores and Eddy, 2006); which translates into an extra cooling requirement of $4 \mathrm{kWh}$ every time a vehicle with a frozen load visits a DC. In the model, it is possible to use variable temperature differences for the different locations. But because our case study is in The Netherlands which is a small country (surface area $41,543 \mathrm{~km}^{2}$ ) with small temperature differences, we assume that the temperature in all locations is $30^{\circ} \mathrm{C}$. This is warmer than the average Dutch temperature, but it is the same as assumptions that were made for previous research (McKinnon et al., 2003; Tassou et al., 2009), such that the results of this Chapter are more convenient for comparison with previous research.

\section{Motive and thermal efficiency}

For the vehicles, we assume that the cooling system is powered by a diesel engine built into the refrigeration unit (Tassou et al., 2009). In a diesel-driven cooling system, chemical energy from the diesel is converted to electrical energy, which is converted to thermal energy (Bell, 2008). For the conversion from chemical to electrical energy $\left(\eta_{e}\right)$, we assume a 
conversion rate of $30 \%$, the same as for conversion from chemical to motive energy $\left(\eta_{m}\right)$ (Bektaş and Laporte, 2011). The Coefficient of Performance (COP), which describes the ratio of heat removed as function of energy supplied, is generally between 0.5 and 1.5 for refrigerated transport of chilled food (at $2^{\circ} \mathrm{C}$ ) (Tassou et al., 2009). The COP can be higher than 1 because the electric power is used to transport energy (heat) instead of converting it. McKinnon et al. (2003) describe that the fuel needed for frozen food transport is $33 \%$ higher than fuel use of chilled food transport. Therefore, if we assume an average COP of 1 for chilled food transport, it is reasonable to assume a COP of 0.67 for frozen food transport. However, data on COPs, or on the efficiency of diesel driven refrigeration systems is hard to find and other authors also state that there is a lack of such data (James et al., 2009).

\section{Conversion of fuel and refrigerant to emissions}

We assume that using 1 liter of diesel causes $2.668 \mathrm{~kg}$ of $\mathrm{CO}_{2}$ emissions, so a fuel-toemission conversion factor $\left(e_{f}\right)$ of 2.668 (Tassou et al., 2009). We assume a $10 \%$ annual refrigerant leakage, which translates into a conversion factor from emissions caused by thermal fuel into emissions caused by thermal fuel and refrigerant leakage $\left(e_{r}\right)$ of 1.21 (Koehler et al., 1997). We assume the energy content of the fuel to be $8.8 \mathrm{kWh} / \mathrm{L}$ (Bektaş and Laporte, 2011).

\section{Setup of calculations}

The case study is divided into four sections. First, we demonstrate the importance of accounting for vehicle load as well as thermal fuel use and refrigerant leakage. Therefore, we compare the results of three different models: (1) the standard VRP model, which minimises total distance, (2) the LDVRP model, which takes into account the load of the vehicle and minimises motive emissions, and (3) the temperature-controlled version of the LDVRP model (TCLDVRP), which minimises total emissions by accounting for both the load of the vehicle and refrigeration. Thus, we compare the VRP, LDVRP and the TCLDVRP models in a realistic setting. Then, we test the effect of different scenarios on the VRP, LDVRP and TCLDVRP models. As some of the parameters used in the models are uncertain, we show how the TCLDVRP model responds to variations in those parameters in a sensitivity analysis. These parameters can vary, but within a day, they are generally stable. Lastly, we test if our model is robust to treat traffic uncertainty. Traffic uncertainty is treated separately from the sensitivity analysis, because in general, the average speeds on certain routes are known. However, within a day, the traffic situations can change. Therefore, we constructed different speed matrices (with different average speeds and standard deviations of the speeds) to represent possible traffic scenarios. Then, we minimised emissions for a fixed speed and then calculated the emissions related to the speed matrices that were designed. These outcomes were compared to the results of minimising emissions considering the speed matrices a priori. This way, it is possible to test the quality of the solutions with uncertain traffic information. The average and standard deviations of the speeds tested in the scenarios are presented together with the results for a clear overview. The models were built and solved in Fico Xpress Mosel version 8.0. 


\subsection{Results}

\section{Comparison of the VRP, LDVRP and TCLVRP models}

Table 2.3 shows the results of the model when the total distance (VRP), the motive energy (LDVRP), and emissions (TCLDVRP; including emissions caused by refrigeration) are minimised for one month of our dataset. The routes are optimised based on the demand on that day. Not all locations have demand on each day. On one of the days, for example, there is demand all locations. For each model the optimal routes are slightly different. VRP: $1-3-7-2-6-9-4-8-1,1-5-10-1$; LDVRP: $1-3-1,1-7-2-6-9-4-8-1,1-$ 10-5-1; and TCLDVRP: $1-3-7-2-6-9-4-8-1,1-10-5-1$. Total distances are not that far apart, but in terms of emissions, the differences are more pronounced.

Table 2.3: Results of optimising the base case VRP, LDVRP and TCLDVRP for one month.

\begin{tabular}{lrrr}
\hline Model & \multicolumn{1}{c}{ VRP } & LDVRP & TCLDVRP \\
\hline Distance $(\mathrm{km})$ & 14531 & 14643 & 14553 \\
Emissions $\left(\mathrm{kg} \mathrm{CO}_{2}\right)$ & 11402 & 11140 & 11119 \\
Costs $(€)$ & 8034 & 8011 & 7970 \\
Time $(\mathrm{h})$ & 225 & 250 & 244 \\
Fuel use $(\mathrm{L})$ & 4028 & 3919 & 3914 \\
Thermal emissions $\left(\mathrm{kg} \mathrm{CO}_{2}\right)$ & 3855 & 4019 & 3976 \\
Motive emissions $\left(\mathrm{kg} \mathrm{CO}_{2}\right)$ & 7547 & 7120 & 7143 \\
\hline
\end{tabular}

Table 2.3 also shows that the VRP model results in the lowest distance and the lowest time. The LDVRP results in the lowest motive emissions. The TCLDVRP model results in the lowest fuel consumption, total costs, and $\mathrm{CO}_{2}$ emissions. The contribution of thermal emissions to total emissions varies between 33,8\% (VRP) and 36.1\% (LDVRP) which is in line with previous research findings (Tassou et al., 2009; Adekomaya et al., 2016).

\section{Scenarios}

Here we test the effect of changing parameters that would e.g. have a different value when the case study would be performed in a different country, a different type of company, or because of technological innovations. The results are shown for 1 month in all tables and figures.

\section{Slope}

Table 2.4 shows the results of the different models for increasing slopes. The following slopes variations were tested: $0.05^{\circ}, 0.2^{\circ}$, and $0.5^{\circ}$. These are the slopes assumed for the arcs directed to the east. But note that a certain positive eastward slope corresponds to a negative slope of the same magnitude in the westwards direction (e.g. $0.1^{\circ}$ for all eastwards nodes corresponds to $-0.1^{\circ}$ for all westwards nodes). In a horizontal displacement of $300 \mathrm{~km}$ (the approximate width of the Netherlands), the slopes tested correspond to a maximum altitude difference of $261.7 \mathrm{~m}, 1047 \mathrm{~m}$, and $2618 \mathrm{~m}$, respectively. Table 2.4 shows the results for the three models and the different slopes. 
Table 2.4: Results of the VRP, LDVRP and TCLDVRP models for increasing slopes. Notation: D, distance; $E$, emissions; $C$, costs; $T$, time; $F$, fuel; $E_{t}$, thermal emissions; $E_{m}$; motive emissions.

\begin{tabular}{|c|c|c|c|c|c|c|c|c|c|}
\hline Slope & \multicolumn{3}{|c|}{$0.05^{\circ}$} & \multicolumn{3}{|c|}{$0.2^{\circ}$} & \multicolumn{3}{|c|}{$0.5^{\circ}$} \\
\hline Model & VRP & LDVRP & TCLDVRP & VRP & LDVRP & TCLDVRP & VRP & LDVRP & TCLDVRP \\
\hline $\mathrm{D}(\mathrm{km})$ & 14531 & 14643 & 14610 & 14531 & 15605 & 14856 & 14531 & 16952 & 16434 \\
\hline $\mathrm{E}\left(\mathrm{kg} \mathrm{CO}_{2}\right)$ & 11479 & 11082 & 11077 & 11767 & 10905 & 10853 & 12345 & 9825 & 9705 \\
\hline$C(€)$ & 8074 & 7980 & 7969 & 8226 & 8135 & 7910 & 8529 & 7779 & 7609 \\
\hline$T(h)$ & 225 & 250 & 248 & 225 & 278 & 256 & 225 & 307 & 298 \\
\hline$F(L)$ & 4057 & 3897 & 3896 & 4165 & 3809 & 3807 & 4381 & 3384 & 3349 \\
\hline $\mathrm{E}_{\mathrm{t}}\left(\mathrm{kg} \mathrm{CO}_{2}\right)$ & 3855 & 4019 & 4006 & 3855 & 4374 & 4091 & 3855 & 4677 & 4523 \\
\hline $\mathrm{E}_{\mathrm{m}}\left(\mathrm{kgCO}_{2}\right)$ & 7624 & 7062 & 7071 & 7913 & 6531 & 6762 & 8490 & 5148 & 5182 \\
\hline
\end{tabular}

Table 2.4 shows that a larger slope increases the differences between the VRP model and the LDVRP model and between the VRP model and the TCLDVRP model considerably. Also the differences between the LDVRP model and the TCLDVRP model are larger than in the base case. The LDVRP model results in lower costs, fuel consumption and $\mathrm{CO}_{2}$ emissions compared with the VRP model, and the TCLDVRP model lowers those outputs even further. For example, using the TCLDVRP model instead of the VRP model for routing temperature-controlled vehicles in an area with a $0.5^{\circ}$ slope can result in a $12 \%$ cost decrease, a $30 \%$ fuel and a $27 \% \mathrm{CO}_{2}$ emission decrease. For the LDVRP and the TCLDVRP, a higher slope is also related to a higher relative contribution of thermal emissions to total emissions: $47 \%$ and $48 \%$, respectively. Moreover, Table 2.4 shows that an increase in steepness can reduce emissions, costs and fuel consumption in case of the LDVRP and the TCLDVRP. This can be explained by Equation (2.5), which calculates motive energy. The first term of this equation is influenced directly by parameter $\alpha_{i j}$, which depends on the slope of the arc. This constant is multiplied by the vehicle weight, including the load, and the distance. The LDVRP and the TCLDVRP models minimise the emissions, which are largely influenced by motive energy requirement. If there is a slope, the optimal routes can be chosen such that, for example, the vehicle is heavily loaded on the downward slopes and lightly loaded on the upward slopes, which saves fuel spent on motive energy.

\section{Cargo weight}

To test the effect of transport of different cargo weights, the demand was kept at the same level but the weight per product was varied. The effects of a changing product weight on the total costs and emissions are shown in Figures 2.1 and 2.2. These Figures show that changing the weight per product, and thus the resulting total cargo weight, changes costs and emissions for all models: an increased weight causes increased fuel consumption and consequently, increased costs and emissions. Still, emissions are lowest for the TCLDVRP in all cases, followed by the LDVRP and the VRP. The TCLVDRP results in the lowest costs in 3 out of 5 cases tested, and the VRP results in the lowest cases in the other two cases. Zachariadis et al. (2015) showed that a higher cargo to empty vehicle weight ratio increased the savings of the LDVRP model compared to using a VRP model. This is not visible in our results, although the LDVRP does always outperform the VRP in terms of emissions. This difference in results can be explained by the fact that in this study, the emissions are not only caused by the weighted distance but also by refrigeration, which 


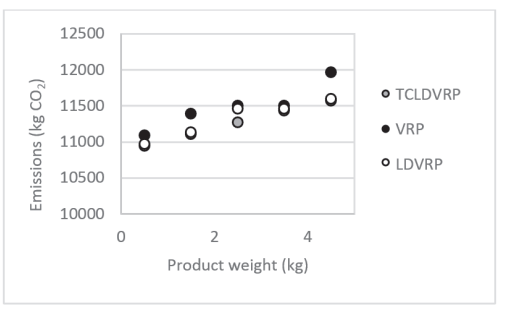

Figure 2.1: Emissions $\left(\mathrm{kg} \mathrm{CO} \mathrm{CO}_{2}\right)$ as a function of the weight per product $(\mathrm{kg})$ for the different models.

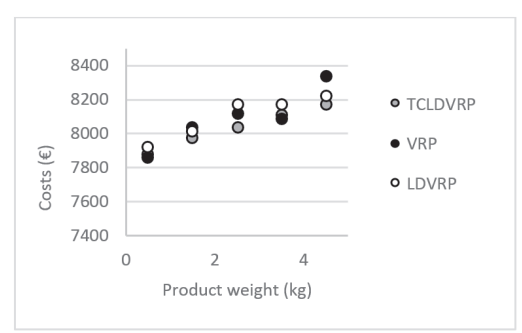

Figure 2.2: Costs (€) as a function of the weight per product for the different models.

the LDVRP does not optimise for.

\section{Temperature inside vehicle}

This case study has focused on frozen food transport, but the models were also tested for chilled transportation $\left(2^{\circ} \mathrm{C}\right)$ and ambient transportation (no cooling). For cooled transportation it was assumed that the COP was 1 (instead of 0.67 for frozen transportation) as those cooling systems are more efficient (Tassou et al., 2009). Running the TCLDVRP model for chilled transportation, resulted in 19\% less emissions than for frozen food transportation. The TCLDVRP solution was still lowest for fuel consumption, $\mathrm{CO}_{2}$ emissions and costs. As can be expected, for ambient temperature transport, the TCLDVRP solution was the same as the LDVRP solution, and that solution caused $32 \%$ less emissions than the frozen transport.

\section{Maximum allowed driving time}

We tested the effect of allowing longer driving times. In the base case, a vehicle driver needs to be back in the depot within 8 hours, and we tested the effects of changing that to 9 and 10 hours. We tested the effect of changing the maximum allowed driving time with the different models (VRP, LDVRP, TCLDVRP) and different indicators (distance, emissions, costs, time and fuel consumption). For all objectives, all indicators (except for the total time) improved.

\section{Sensitivity analysis}

The sensitivity of the TCLDVRP model was tested for different road-and vehicle-specific parameters. The results are shown for one month for all figures in this section.

\section{Speed and coefficient of performance}

To test the effect of speed and Coefficient of Performance (COP), we tested the effects of applying the TCLDVRP model on average matrix speeds between 39.4 and $78.7 \mathrm{~km} / \mathrm{h}$ for a COP of 0.67 (base case) and a COP of 2 (technological improvement case). The results are shown in Figures 2.3 and 2.4. 


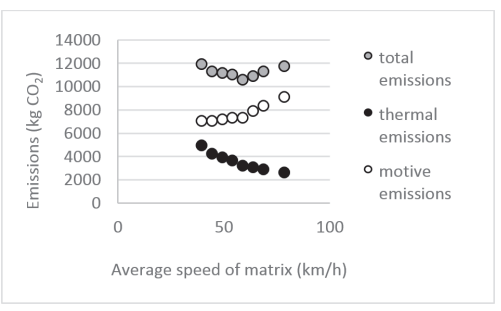

Figure 2.3: Emissions $\left(\mathrm{kg} \mathrm{CO}_{2}\right)$ as function of average matrix speed for a COP of 0.67 and using the TCLDVRP model.

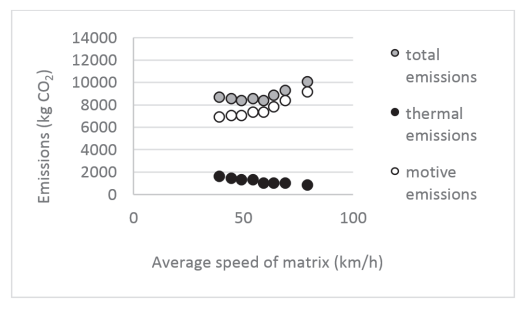

Figure 2.4: Emissions $\left(\mathrm{kg} \mathrm{CO}_{2}\right)$ as function of average matrix speed for a COP of 2 and using the TCLDVRP model.

The LDVRP (motive emissions) model results in an optimal speed of around $40 \mathrm{~km} / \mathrm{h}$, which is in line with findings from previous research (Jabaliet al., 2012). However, for both COPs, the optimum speed for the TCLDVRP is around $60 \mathrm{~km} / \mathrm{h}$. This shows that taking the thermal energy requirement into account can not only change the optimal route, it can also change the optimal speed. Figures 2.3 and 2.4 show that a higher COP results in a lower total emissions. Also, the higher the COP, the more the total emissions depend on the emissions caused by motive energy and the closer the TCLDVRP solution will be to the LDVRP solution. Previous research suggested that there is a clear trade-off between travel time and greenhouse gas emissions (Aziz and Ukkusuri, 2014), which can hamper implementation of green solutions. These results show that for temperature-controlled transportation, optimal speed increases, which may lead to a higher chance of implementation of the emission-minimising routes.

\section{Heat transfer coefficient}

The higher the heat transfer coefficient, the higher the fuel consumption as a consequence of thermal energy. In practice, the heat transfer coefficient can increase because of ageing of the vehicle.

\section{Distance}

As the distance between the different nodes increases, all outputs increase, but the relative difference between the optimal solutions of the different models stays within the same range. Cenerally the total distance resulting from the optimisation of the TCLDVRP model, is in between the total distance resulting from optimising the LDVRP and the VRP model.

\section{Temperature outside vehicle}

As the literature that we base most of our assumptions on assume an ambient temperature of $30^{\circ} \mathrm{C}$, this is what this Chapter also assumes as base case situation (Tassou et al., 2009; Bektaş and Laporte, 2011). We also tested the effects of lower ambient temperatures. As can be expected, for all three models emissions, costs and fuel use improved with a decreasing outside temperature. Lower temperatures result in smaller differences between the LDVRP and the TCLDVRP. The VRP also shows improved results when lower- 
ing outside temperature but the improvements are smaller compared to the LDVRP and the TCLDVRP. For example, changing the outside temperature from $30^{\circ} \mathrm{C}$ to $10^{\circ} \mathrm{C}$ results in an emission decrease of around 30\% for the TCLDVRP and the LDVRP and for a 10\% decrease for the VRP.

\section{Traffic uncertainty}

Traffic uncertainty is taken into account by letting the model minimise emissions based on a fixed speed but still calculating the effects of different scenarios of the actual speed matrix on emissions and costs. Table 2.5 shows the traffic uncertainty scenarios tested as well as the difference of those scenarios with the optimal solution for emissions with full information on the speed matrix, the optimality gap.

Table 2.5: Traffic uncertainty scenarios and resulting optimality gaps. Symbols: $\mu\left(v_{w}\right)$, average speed to the west; $\sigma\left(v_{w}\right)$, standard deviation speed to the west; $\mu\left(v_{e}\right)$, average speed to the east; $\sigma\left(v_{e}\right)$, standard deviation speed to the east.

\begin{tabular}{ccccccc}
\hline $\begin{array}{c}\text { Sensitivity } \\
\text { scenario }\end{array}$ & $\mu\left(v_{w}\right)$ & $\sigma\left(v_{w}\right)$ & $\mu\left(v_{e}\right)$ & $\sigma\left(v_{e}\right)$ & Optimality gap \\
emissions (\%) & costs (\%) \\
\hline 0 & 47.5 & 3 & 62.5 & 3 & 0.66 & 0.00 \\
1 & 38 & 3 & 50 & 3 & 0.10 & 0.51 \\
2 & 42.75 & 3 & 56.25 & 3 & 0.31 & 0.00 \\
3 & 52.25 & 3 & 68.75 & 3 & 1.72 & 0.00 \\
4 & 57 & 3 & 75 & 3 & 2.96 & 1.09 \\
5 & 47.5 & 1 & 62.5 & 1 & 1.11 & 0.00 \\
6 & 47.5 & 5 & 62.5 & 5 & 1.02 & 0.00 \\
7 & 47.5 & 7 & 62.5 & 7 & 1.64 & 0.00 \\
8 & 55 & 1 & 55 & 1 & 0.01 & 0.00 \\
9 & 55 & 3 & 55 & 3 & 0.09 & 0.00 \\
10 & 55 & 5 & 55 & 5 & 0.05 & 0.00 \\
11 & 55 & 7 & 55 & 7 & 0.27 & 0.00 \\
\hline
\end{tabular}

Table 2.5 shows that traffic uncertainty can cause the TCLDVRP model to not always be optimal in terms of emissions. For all instances tested however, the TCLDVRP solution did not deviate more than $3 \%$ from the minimum emissions with full information on the speed matrix. In terms of costs, the TCDLVRP at its maximum $1.1 \%$ away from the lowest cost solution.

\subsection{Discussion and Conclusions}

This Chapter proposes an extended version of the LDVRP model, which considers emissions caused by refrigeration into account to optimise routing for temperature-controlled transportation, i.e. TCLDVRP. Refrigeration increases the fuel use of the vehicle and it causes leakage of refrigerant, which both increase emissions compared to ambient transportation. Minimising emissions while accounting for refrigeration emissions can result in different optimal routes compared with minimising distance (VRP) or minimising motive energy (LDVRP). Moreover, the TCLDVRP model results in higher optimal speed compared with previous research on fuel or emission minimisation (Bektaş and Laporte, 2011; Jabali et al., 2012; Aziz and Ukkusuri, 2014). The TCLDVRP model improves the en- 
vironmental and economic performance of temperature-controlled road transportation by minimising both emissions caused by motive energy, thermal energy and refrigerant leakage. We have demonstrated that the TCLDVRP model results in savings in emissions and costs for temperature-controlled road transportation. Our case study focused on frozen food transportation but the model can also be used to optimise cooled or ambient transportation by adjusting the temperature and the COP. Furthermore, by changing the area through which heat exchange takes place, the model can be used for vehicles with compartments at different temperatures. This case study was done with frozen food but the findings can also be used in of fresh food, medication, flowers, and other temperature sensitive products.

To calculate refrigeration emissions, we considered two types of emissions: those caused by fuel use of the refrigeration unit, and those caused by refrigerant leakage. The fuel use of the refrigeration unit was assumed to depend on energy requirements caused by heat entering through the vehicle wall during driving, and heat entering when the door opens. To improve the accuracy of the calculations, more thermal processes could be taken into account. For example, for cooled and ambient transportation, fruit and vegetables can produce heat by ripening, which can increase the cooling requirement. On the other hand, heavy cooled loads can have effects such as self-insulation, which can decrease the cooling requirement. Accounting for such processes will result in non-linear models that will require advanced heuristics algorithms to be solved in an acceptable time (Demir et al., 2014). Such algorithms might result in sub-optimal solutions and consequently the gain of accuracy with refrigeration emissions can be counterbalanced.

This Chapter focuses on routing strategies to reduce emissions. Literature however also suggests a more long term approach to reduce emissions: by logistics cooperation. For example, if companies have the same delivery region and they decide to collaborate on their deliveries they have the opportunity to organise their deliveries in a more efficient way such that both costs and emissions are saved (Cruijssen et al., 2007b; Vanovermeire et al., 2014; Cuajardo and Rönnqvist, 2016). The TCLDVRP model could be of use in this research area, as it can be used to quantify the benefits of collaborative refrigerated transportation.

In our communication with and observation of practitioners, we found that there is probably improvement possibilities by changing ways of working. For example, the docks at which vehicles connect to the temperature controlled distribution centres (DC) are not always airtight. This causes warm air to enter the DC and it thus increases the energy use in the DC. In other cases, we saw that drivers already opened their vehicle hoping that they would have to wait less to be connected to the dock. This behaviour highly increases the work that the cooling engine has to do and sometimes even caused the engine to overheat. Also, this sudden temperature increase can severely influence the food quality. For example, frozen foods that defreeze a bit and then freeze again will get a different structure with some ice formation. This will lead to decreased sales and more waste. A first step for companies to reduce emissions and costs in temperature controlled transportation would be to critically evaluate these kind of practises. 
We showed that it was necessary to account for emissions caused by refrigeration energy as well as refrigerant leakage in the minimisation of emissions from temperature controlled transportation. We extended a LDVRP model to account for these effects, and we applied this model on a frozen food transportation case to shown the effects of applying this model in practise. Our results confirm that emissions caused by refrigeration are responsible for around $40 \%$ of total emissions from temperature controlled transportation. Also, we show that these effects are so considerable that the can change optimal routing decisions. An improvement in the conversion efficiency of fuel to both thermal and motive energy is a very potent way of reducing fuel consumption, costs and emissions in road transportation. Improvements in efficiency can be achieved through new technologies that focus on improving energy efficiency in (temperature-controlled) vehicles, such as cooling with liquid nitrogen (Garlov et al., 2002), using cryogenic cooling systems (Tassou et al., 2009), driving with liquefied natural gas vehicles (Kumar et al., 2011) or with electric vehicles (Pelletier et al., 2016). The proposed model for temperature-controlled transportation routing problem can be used to quantify the benefits of these new technologies. 


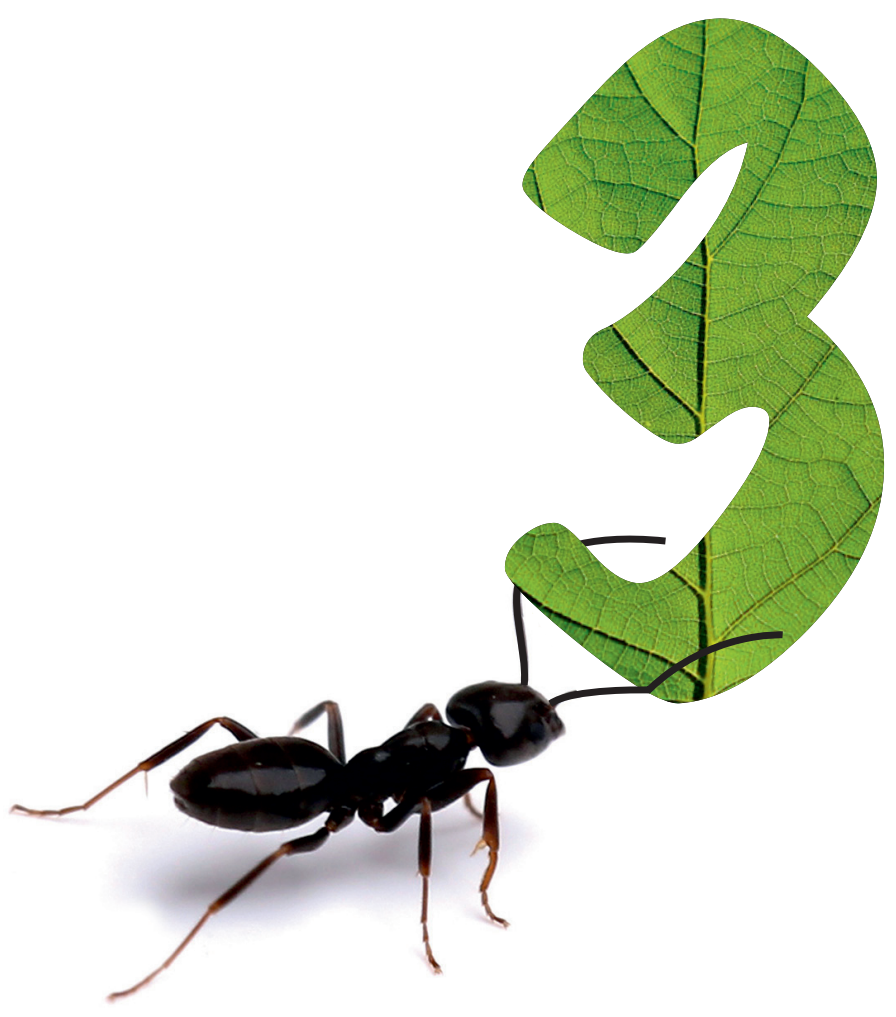




\title{
Chapter 3
}

\section{The quality-driven vehicle routing problem: Model and application to a case of cooperative logistics}

\begin{abstract}
Inefficient road transportation causes unnecessary costs and emissions, especially in fresh food transportation, where temperature control is used to guarantee product quality. On a route with multiple stops, the quality of the transported products could be negatively influenced by the door openings and consequent temperature fluctuations. In this study, we quantify the effects of multi-stop transportation on food quality. To realistically model and quantify food quality, we develop a time-and temperature-dependent kinetic model for a vehicle routing problem. The proposed extensions of the vehicle routing problem enable quantification of quality decay on a route. The model is illustrated using a case study of cooperative routing, and our results show that longer, multi-stop routes can negatively influence food quality, especially for products delivered later in the route, and when the products are very temperature-sensitive and the outside temperature is high. Minimising quality loss results in multiple routes with fewer stops per route, whereas minimising costs or emissions results in longer routes. By adjusting driving speed, unloading rate, cooling rate, and by setting a quality threshold level, the negative quality consequences of multi-stop routes can be mitigated.
\end{abstract}

This chapter is based on: Stellingwerf, H.M., Groeneveld, L.H.C., Laporte, G., Kanellopoulos, A., Bloemhof, J.M., Behdani, B. (under review). The quality-driven vehicle routing problem: Model and application to a case of cooperative logistics. 


\subsection{Introduction}

The Vehicle Routing Problem (VRP) has been traditionally modelled for minimising distance or the costs of routing product flows to multiple locations. As a new variant of VRP, the green VRP has been developed to also account for emissions (Bektaş and Laporte, 2011). Compared with ambient transportation, the transportation of frozen and fresh food products is costlier and more polluting due to the energy required for cooling. To address this, Stellingwerfet al. (2018a) have extended a model based on the VRP to account for the effects of temperature control on costs and emissions in fresh and frozen food logistics. In fresh food logistics the temperature fluctuations resulting from the increased number of stops on a route may further influence the quality of the products transported. Also, transporting multiple products with a different optimal temperature, can be challenging with substantial consequences for the product's quality. Therefore, temperature control is an essential factor in the distribution of food products (Akkerman et al., 2010). Keeping perishable foods cooled or frozen along the food supply chain is vital to guarantee food safety, manage food waste and ensure good quality of the final product. Therefore, it is necessary to consider the influence of temperature on food quality aspects in VRP modelling.

This Chapter introduces a VRP that explicitly considers the quality decay in transportation planning, both in the constraints and in the objective function. Using the presented model, we compare several objectives including minimising costs, emissions, and quality loss. We then study the effect of transporting different products with different optimal temperatures in one vehicle on the resulting product quality. We also test the effect of other parameters.

We illustrate the model using the case of seven Dutch supermarket chains that cooperatively buy their products in order to obtain a lower price. The supermarket chains consider intensifying their cooperation by also transporting their products together, in order to save transportation costs and emissions. The partners wish to have a quantification of the potential risks and benefits related to quality decay, costs and emissions to decide whether it compensates for the information that they need to share with each other in a cooperative context. Logistics cooperation has been shown to be a feasible methodology to decrease both cost and emissions during transportation of food products (Vanovermeire et al., 2014; Pérez-Bernabeu et al., 2015; Quintero-Araujo et al., 2017; Mittal et al., 2018). Most of these studies found that cooperation can result in cost reductions (Cruijssen et al., 2007b; Quintero-Araujo et al., 2017), and some have also identified emission reductions in addition to cost reductions (Pérez-Bernabeu et al., 2015; Stellingwerfet al., 2018b). For a recent overview of the optimisation of different forms of cooperation, we refer to Defryn and Sörensen (2018). However, a cooperative route will result in an increased number of stops, which may negatively affect food quality. With our Quality Driven VRP (QDVRP), we can also assess the effect of logistics cooperation on food quality, costs, and emissions.

The remainder of this Chapter is structured as follows. In Section 3.2 we discuss food quality and how it has been modelled, in Section 3.3 we mathematically formulate the prob- 
lem, in Section 3.4 we show and discuss the results, and in Section 3.5 we conclude this Chapter and suggest future research directions.

\subsection{Modelling food quality}

In the logistics literature, quality decay has been modelled using several approaches. We categorise these and discuss each of the categories in the following subsections.

\section{Modelling quality considering product age and remaining shelf life}

A common method to handle product quality in logistics modelling is to consider a fixed shelf life for perishable items. To approximate freshness, Amorim and Almada-Lobo (2014) have quantified the remaining shelf life as a percentage of the initial shelf life in a multiobjective VRP. They compared two objective functions: cost minimisation and maximisation of the an average shelf life. Stellingwerfet al. (2018b) proposed an inventory-routing problem (IRP) model that minimises costs, emissions, or a linear combination of both objectives, and applied it to a case of temperature-controlled food distribution. After finding the optimal routing and inventory plan, the resulting average product age upon leaving the distribution centres (DCs) was calculated in days. Likewise, Soysal et al. (2018) proposed a green IRP for perishable products. Each product was assumed to have a fixed shelf life, after which it would go to waste, incurring a penalty cost.

These studies provide a way of integrating shelf life or food quality into routing models, but they do not consider how external factors, such as temperature, affect the products during transportation. Modelling quality decay (which is dependent on external factors) instead of shelf life (which often is a predetermined date) should yield a more realistic way of modelling food quality.

\section{Modelling quality with temperature-independent quality decay}

To model quality decay, some studies have used a temperature-independent decay function. Thus Ambrosino and Sciomachen (2007) accounted for quality in their VRP by imposing a maximum number of stops on the routes of the vehicles if they carried frozen products. A binary variable was used to decide whether a certain vehicle would move only dry products or also frozen products. If frozen products were transported, a constraint to limit the maximum number of stops was activated.

Osvald and Stirn (2008) studied decay during transportation using a VRP with time windows and time-dependent travel times. They assumed that quality is linearly related to time and assigned a quality starting level that decreases over time for each load. They considered the effect of delays on quality and compared a standard cost-minimisation model with a cost-minimisation model including a penalty cost for product loss due to quality decay. Their study showed that by considering quality decay in the optimisation model, up to $40 \%$ of cost savings could be realised. 
Chen et al. (2009) considered a quality decay function in their production scheduling and vehicle routing problem, where total profit was maximised. The study showed that a higher decay rate leads to a lower profit, and that deterioration could be reduced by using more vehicles. However, the latter also leads to an increase in transportation cost.

\section{Modelling quality with temperature-dependent quality decay}

Hsu et al. (2007) modelled the expected loss of inventory due to quality decay in a stochastic VRP with time windows. They considered decay to be stochastic: the higher the demand per stop, the longer the door-opening time, the higher the temperature in the vehicle, and the higher the chance of spoilage of the products transported. The goal of the model was to minimise cost, in which spoilage was part of the inventory cost. Aung and Chang (2014) used a similar model with a different objective function to determine the optimal temperature for a range of products.

The studies just discussed show that shelf life and food quality have been considered in supply chain and logistics literature. However, they do not consider external factors such as temperature in the quality decay function.

\section{Modelling quality with kinetics}

Kinetic modelling is used to describe the direction and speed of different kind of reactions and it is often used to model changes in food products, for example as a function of temperature. This method is the basis for modelling quality in this paper; therefore, we now describe the main principles of kinetics modelling for quality decay of perishable products.

According to Van Boekel (2008) there exist four main types of reactions that can cause quality-related changes in food products: (i) chemical reactions, which often relate to oxidation reactions; (ii) microbial reactions; (iii) biochemical reactions, caused or catalysed by enzymes naturally present in foods; and (iv) physical reactions, such as coalescence, sedimentation and texture changes. These changes can be captured by mathematical models containing kinetic parameters. For reactions in food, zero and first order reactions are relevant. Zero order reactions happen at a constant speed, while in first order reactions, the speed changes linearly over time. In a zero order reaction, decay happens linearly over time. In a first order reaction, decay happens exponentially over time. This order type can be empirically derived, but in general degradation of quality attributes of fruits and vegetables follow a zero order reaction (Rong et al., 2011). Quality degradation that is mainly dependent on microbial growth (e.g. meat and fish) generally follow a first order reaction rate.

For temperature-dependent reaction kinetics, the Arrhenius law (Van Boekel, 2008) is very often used to predict the rate constant $\kappa$ of a reaction based on absolute temperature $T$ :

$$
\kappa=\kappa_{0} \exp \frac{-E_{a}}{R T}
$$


where $\kappa$ is the rate constant $\left(\mathrm{s}^{-1}\right.$ for a first order reaction and $\mathrm{mol} / \mathrm{L} / \mathrm{s}$ for a zero order reaction), $\kappa_{0}$ is the pre-exponential factor $\left(\mathrm{s}^{-1}\right.$ for a first order reaction and $\mathrm{mol} / \mathrm{L} / \mathrm{s}$ for a zero order reaction), $E_{a}$ is is the activation energy (Joule/mol), $R$ is the gas constant ( 8.3145 Joule/mol/K), and $T$ is the temperature in degrees Kelvin (K). Different quality attributes at different temperatures for a certain type of food can be empirically estimated for the Arrhenius equation.

Mishra et al. (2016) empirically estimated the parameters in the Arrhenius equation for the quality indicators appearance, wilting, browning, and off-odour for fresh-cut iceberg lettuce, fresh-cut romaine lettuce, and fresh-cut chicory. In their study, the decay was modelled as a percentage of initial quality lost.

Rong et al. (2011) focused on integrating a food quality model in a logistics model. They used the Arrhenius equation to describe an ad hoc overall quality (not related to a specific real quality attribute), which they set at 100 at the beginning of the supply chain and then lowered as the product moved down the chain. They modelled food quality degradation through a mixed integer linear programming (MILP) model by combining existing food quality decay models and logistics models. Quality was modelled using

$$
\frac{d q}{d t}=\kappa q^{n},
$$

where $d q$ is the change in quality (dimensionless), $d t$ is the change in time (days), $\kappa$ is the degradation rate $\left(\right.$ days $^{-1}$ ), which was approximated using the Arrhenius equation (Equation 3.1), and $n$ indicates the order or the reaction ( 1 for a first order reaction, and 0 for a zero order reaction).

In the model of Rong et al. (2011), products deteriorate by a given amount in each period such that the model can track the quality degradation over time. Each product starts with a given quality which decreases each period based on the time and temperature exposure. When the quality level is lower than the predetermined minimum, the product goes to waste.

Table 3.1 summarises the literature on modelling quality in food logistics, as described in this section. 


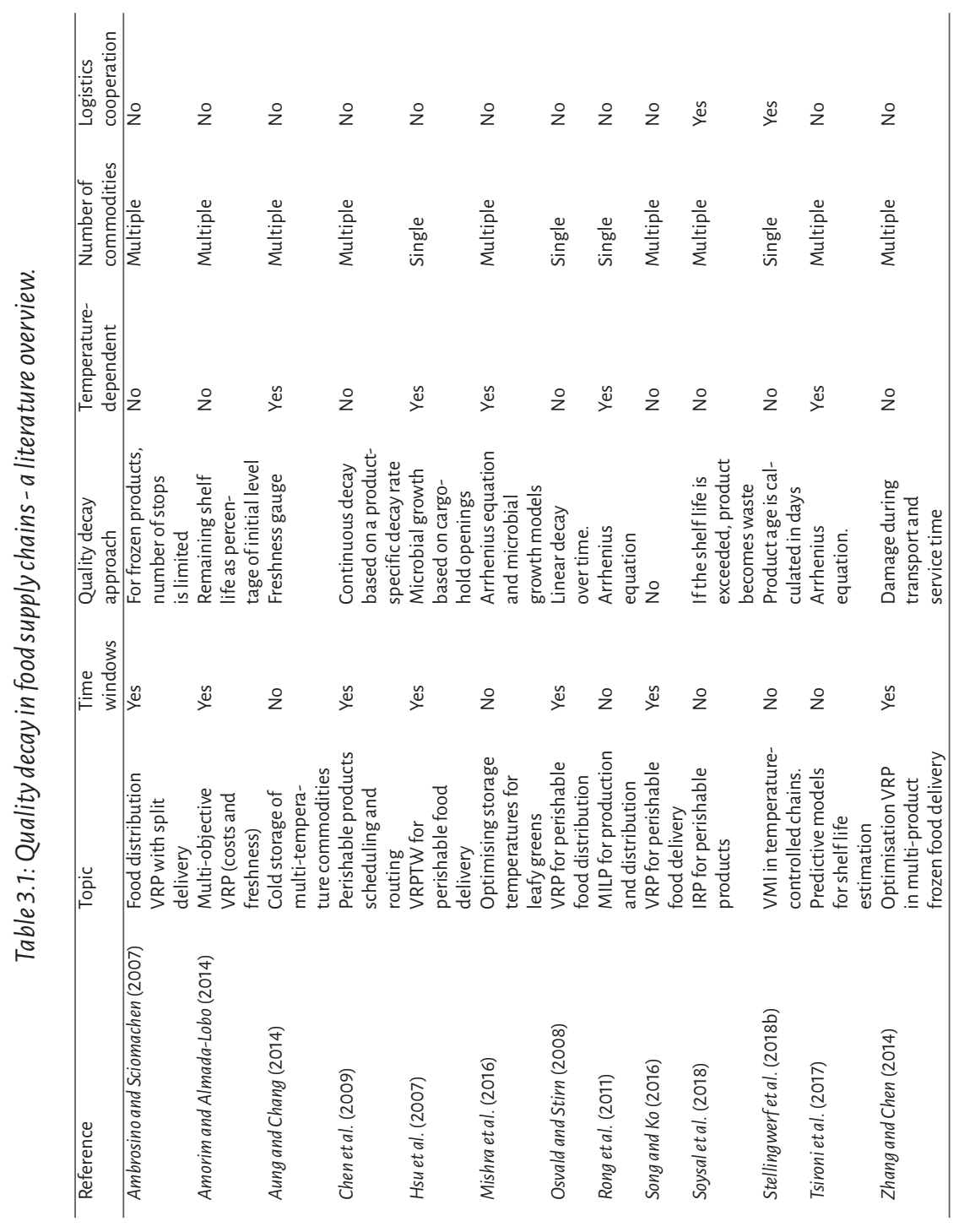




\subsection{Formal problem description and mathematical formulation}

In order to model the effect of cooperative routing of the quality of fresh products, we use the temperature-controlled load dependent VRP model of Stellingwerfet al. (2018a). The objective functions of this model are cost and emissions minimisation. Here we add a quality decay minimisation objective. We extend this model to account for quality decay at each stop and on each arc. We measure quality decay as in Rong et al. (2011) and we make the service time demand-dependent based on the study of Hsu et al. (2007). We adjust it to account for multiple products, with their own optimal temperature and their own Arrhenius parameters, based on Mishra et al. (2016). In addition, we use the total cost and total emissions calculations of Stellingwerfet al. (2018a).

The problem under consideration is NP-hard since it encompasses the VRP which is known to be NP-hard. Here, we provide the formulation of a quality driven VRP, which is used to model cooperative routes and the effect on food quality, cost and emissions. A summary of all notations (including the units of all variables and parameters) used is given in the Appendix (Tables A.1-A.4).

Let $G=(V, A)$ be a directed graph in which $V=\{0,1, \ldots, n\}$ is the set of nodes. The CDC is located at vertex $0, V \backslash\{0\}$ is the set of DCs, and $A=\{(i, j): i, j \in V, i \neq j\}$ is the set of arcs. With every $\operatorname{arc}(i, j)$ is associated a non-negative distance $c_{i j}$. We define $p$ as an index for the set $P$ of products with rate constant $\kappa_{0}^{p}$, activation energy $E_{a}^{p}$, and optimal temperature $T_{\text {ref }}^{p}$.

The demand of product $p$ at each DC $i$ is given by $q_{i}^{p}$. The load inside every vehicle has a changing quality level denoted as $Q_{i j}^{k p}$. At the starting CDC, all loads have an initial quality of $Q_{0}^{p}$. There exists a set of $K$ identical vehicles indexed by $k$ of capacity $L^{k}$ with a curb (empty) weight of $L_{0}^{k}$. The speed driven on $\operatorname{arc}(i, j)$ is denoted by $v_{i j}$. We denote by $d$ the maximum travelling time of one driver, and by $s_{i}$ the service time the vehicle spends at node $i$.

The following decision variables are used:

- $x_{i j}^{k}$ is a binary variable equal to 1 if and only if vehicle $k$ drives from node $i$ to node $j$

- $w_{i j}^{k p}$ is the total weight of product $p$ carried, from node $i$ to node $j$ by vehicle $k$

- the binary decision variable $z_{i j}^{k}$ is used in a set of constraints to control the maximum cooling time on an arc

- $D_{i j}^{k p}$ is the total decay at an arc

- $D_{i j, v}^{k p}$ is the decay during cooling time (at a variable temperature) at an arc

- $D_{i j, f}^{k p}$ is the decay at a fixed temperature on an arc

- $Q_{i j}^{p m}$ is the quality level arriving at node $j$ from node $i$ of product $p$ with vehicle $k$

- $s c_{i j}^{k}$ is the cooling time of vehicle $k$ on arc $i j$

- $Z$ is a variable to minimise the maximum quality decay 


\section{Calculation of quality decay}

Over time, fresh food products decay in temperature-dependent fashion. When fresh food is transported, the temperature in the vehicle is set to the optimum. When there are products with different optimal temperatures, a temperature that is acceptable for all products is chosen. We assume that the outside temperature is higher than the temperature set in the vehicle, which means that the load needs to be cooled to reach the desired temperature. We also assume that the products need to be cooled but not frozen. After precooling, the temperature of the product inside transportation vehicles remains quite stable. However, temperatures can rise up to $10^{\circ} \mathrm{C}$ in just minutes during operations such as loading and unloading the vehicle (Mercier et al., 2017). A cooled food product can be subjected to up to fifty door openings per transportation run (James et al., 2006). For fresh food (unfrozen), the closer the product is to its optimal temperature, the slower it decays. In reality, when the temperatures becomes lower than the optimal temperature, freezing damage can occur. However, this freezing temperature is not considered in this model. When a vehicle visits a delivery point, the vehicle door opens, and consequently the temperature of the air in the vehicle and the products in the vehicle will increase. When there are multiple stops, the products that are still in the vehicle after a delivery has been made, are faced with this temperature increase. The cooling engine of the vehicle will start working to cool the vehicle again.

To include quality in a VRP model, we model two decays in the chain; the quality decay in the links and quality decay in the nodes. Both decay processes are influenced by temperature variations. We define quality decay of a product on a route as the sum of both types of decay: the quality decay at the arcs it traversed and the quality decay at the nodes it passed before arriving at its destination:

$$
D=\sum_{i \in V} \sum_{k \in k} \sum_{p \in P} D_{i}^{k p}+\sum_{i \in V} \sum_{j \in V} \sum_{k \in K} \sum_{p \in P} x_{i j}^{k} D_{i j}^{k p},
$$

where $D$ is the total quality decay, $D_{i}^{k p}$ is the quality decay of product $p$ at node $i$ in vehicle $k, D_{i j}^{k p}$ is the quality decay of product $p$ at arc $i j$ in vehicle $k$.

\section{Quality decay at a node}

The quality at a node is calculated using the Arrhenius equation (Tsironi et al., 2017) assuming a zero order reaction for the products:

$$
D_{i}^{k p}=\kappa_{0}^{p} s_{i} \exp \left[\frac{-E_{a}^{p}}{R}\left(\frac{1}{T_{i}^{k p}}-\frac{1}{T_{r e f, i}}\right)\right]
$$

where $s_{i}$ is the demand-dependent service time at node $i, T_{i}^{k p}$ is the temperature of the products in vehicle $k$ at node $i, E_{a}^{p}$ is the activation energy of product $p$.

The service time at a node is calculated as follows: 


$$
s_{i}=\sum_{p \in P} q_{i}^{p} t_{s}
$$

where $q_{i}$ is the demand at node $i$, and $t_{s}$ is the unloading rate. We use an approximation based on the study of (Tso et al., 2002) to calculate the temperature change of the products caused by the opening of the door during service time. The temperature change happens in a two-step process described in (3.6)-(3.8).

First, the air temperature of the vehicle is calculated:

$$
\begin{aligned}
& T_{i}^{k}=\min \left\{T_{a} ; 0.5 s_{i} f\left(T_{a}-T_{0}\right)+T_{0}\right\} i \in V^{\prime}, k \in K, \\
& T_{0}^{k}=T_{0} \\
& k \in K,
\end{aligned}
$$

where $T_{i}^{k}$ is the air temperature of vehicle $k$ at the end of the service time at node $i, T_{0}^{k}$ is the air temperature of vehicle $k$ when it leaves the CDC, $T_{a}$ is the ambient temperature, $f$ is the speed of the temperature increase, which is assumed to be $0.0027 \mathrm{~K} / \mathrm{s}$ based on the measurements of Tso et al. (2002), $T_{0}$ is the temperature that is set, which is called the goal temperature in the rest of this Chapter. The factor of 0.5 is used to account for the first step of a two-step heating process.

Then, the product temperature is calculated:

$$
T_{i}^{k p}=\min \left\{T_{i}^{k} ; 0.5 s_{i} f\left(T_{i}^{m}-T_{0}\right)+T_{0}\right\},
$$

where $T_{i}^{k p}$ is the temperature of the products in vehicle $k$ at the end of the service time at node $i$. The factor of 0.5 is used to account for the second step of a two-step heating process. For the $\mathrm{CDC}$, we assume no unloading time, and consequently, the air and product temperature will equal the goal temperature.

\section{Quality decay at an arc}

After visiting a node, the vehicle closes and the engine can start cooling the load. When the goal temperature is reached, the engine keeps the temperature at the goal temperature. The quality decay at an arc can thus be divided in two sections: first, there is decay while the temperature decreases from the after-opening temperature, and then there is decay during the rest of the arc traverse, at a fixed temperature:

$$
D_{i j}^{k p}=D_{i j, v}^{k p}+D_{i j, f}^{k p},
$$

where $D_{i j}^{k p}$ is the decay at an arc, $D_{i j, v}^{k p}$ is the decay during cooling time at an arc ( $v$ for variable temperature), and $D_{i j, f}^{k p}$ is the decay during the rest of the arc crossing time ( $f$ for fixed temperature). The decay is dimensionless.

The decay of product $p$ during cooling time at the arc is calculated as 


$$
D_{i j, v}^{k p}=\kappa_{0}^{p} s c_{i j}^{k} \exp \left[\frac{-E_{a}^{p}}{R}\left(\frac{1}{0.5\left(T_{i}^{k}+T_{0}\right)}-\frac{1}{T_{r e f}^{p}}\right)\right],
$$

where $s c_{i j}^{k}$ is the cooling time of vehicle $k$ on arc $i j, T_{r e f}^{p}$ is the reference (optimal) temperature of product $p$. Since the cooling time is dependent on the weight of the load, which is a decision variable, its calculation is given together with the other constraints (see Equations (3.47) - (3.51)).

When the vehicle temperature is down to its optimal level, the rest of the arc is crossed at a stable, i.e. the goal or optimal temperature. The decay at the arc when the temperature is stable is calculated as

$$
D_{i j, f}^{k p}=\kappa_{0}^{p}\left(\frac{x_{i j}^{k} c_{i j}}{v_{i j}}-s c_{i j}^{k}\right) \exp \left[\frac{-E_{a}^{p}}{R}\left(\frac{1}{T_{0}}-\frac{1}{T_{r e f}}\right)\right],
$$

where $\kappa_{0}$ is the rate constant, and $\frac{x_{i j}^{k} c_{i j}}{v_{i j}}$ is used to calculate the time spent on arc $i j$.

\section{Minimising the maximum decay}

For one company delivering food to multiple outlets, it makes sense to minimise total decay. However, for a cooperative, the different partners might find it more important that there is not too much difference between the quality they receive.

Therefore, we also calculate the maximum decay $m$ as

$$
\begin{aligned}
& \sum_{i \in V, j \in V, k \in K, p \in P} D_{i j, r}^{k p}=Q_{0}^{p}-\frac{Q_{i j}^{k p}}{Q_{0}^{p}}, \\
& D_{i j, r}^{k p} \leq Z
\end{aligned}
$$

where $D_{i j, r}^{k p}$ is the relative decay of a product $p$ on arc $i j$ in vehicle $k$. In constraint (3.13) $Z$ is defined as the maximum level of decay of all products arriving at all nodes. Objective (3.29) minimises the maximum decay $Z$.

\section{Fuel and emissions calculation in ambient transportation}

Studies have shown that the fuel use in ambient transport is linearly related to the motive power requirement (Barth and Boriboonsomsin, 2009; Bektaş and Laporte, 2011). The latter depends on the weight carried, the slope of the road, the distance travelled and the vehicle speed. The motive power $P_{i j}$ on $\operatorname{arc}(i, j)$ can be approximated as

$$
P_{i j}=\alpha_{i j}\left(w_{i j}^{k p}+L_{0}^{k}\right) c_{i j}+\beta v_{i j}^{2} c_{i j},
$$

where $\alpha_{i j}$ is the arc-specific constant, $L_{0}^{k}$ is the curb weight of vehicle $k$, and $\beta$ is the vehicle-specific constant. Equations (3.15) and (3.16) show how these constants are calculated:

$$
\alpha_{i j}=a+g \sin \theta_{i j}+g C_{r} \cos \theta_{i j},
$$


where $a$ is the acceleration of the vehicle $\left(\mathrm{m} / \mathrm{s}^{2}\right), g$ is the gravitation constant $\left(\mathrm{m} / \mathrm{s}^{2}\right), \theta_{i j}$ refers to the average slope on arc $i j$ (degrees), $C_{r}$ is the rolling resistance (dimensionless). The vehicle-specific constant is calculated as

$$
\beta^{m}=0.5 C_{d} A \rho,
$$

where $C_{d}$ is the drag coefficient $A$ is the frontal area of the vehicle and $\rho$ is the air density.

In (3.17) fuel use for ambient transport $\left(f_{m}\right)$ is calculated by summing up the power requirements for all routes and converting those into fuel use. This is achieved by dividing the power by $3.6 \times 10^{6}$ to convert a Joule (]) to a kilowatt-hour ( $\mathrm{kWh}$ ), by the chemical to motive energy conversion efficiency $\left(\eta_{m}\right)$, and by the energy content of the fuel $\left(P_{f}\right)$ :

$f_{m}=\left(\sum_{i \in V} \sum_{j \in V} \sum_{k \in K} \sum_{p \in P} \alpha\left(w_{i j}^{k p}+L_{0}\right) c_{i j}+\sum_{i \in V} \sum_{j \in V} \sum_{k \in K} \beta c_{i j} x_{i j}^{k t} v_{i j}^{2}\right) \frac{1}{3.6 \times 10^{6} P_{f} \eta_{m}}$.

The emissions from ambient transport are linearly related to fuel use:

$$
E_{m}=f_{m} e_{f}
$$

where $e_{f}$ is the emissions factor which converts fuel use into $\mathrm{CO}_{2}$ emissions, $E_{m}$ are the $\mathrm{CO}_{2}$ emissions of ambient transport, and $w_{i j}^{k p}$ is the weight of product $p$ carried from node $i$ to $j$ with vehicle $k$.

\section{Fuel and emissions calculation in refrigerated transportation}

Stellingwerfet al. (2018a) have approximated the cost, the fuel consumption and the emissions of refrigerated transport, so that the impact of temperature controlled transport can be estimated in route optimisation models. In refrigerated transport, fuel is used both for motive power and for keeping the temperature of the load at the right level. The energy used for temperature control depends on the heat that enters through the vehicle wall while it drives, and on the heat that enters the vehicle when the door opens. The heat entering through the wall is calculated as

$$
H_{w}=\frac{\sum_{i \in V^{\prime}} \sum_{j \in V^{\prime}} \sum_{k \in K} x_{i j}^{k} c_{i j} U S^{k} \Delta T}{3.6 \times 10^{6} v_{i j}},
$$

where $x_{i j}^{k} c_{i j} / v_{i j}$ is used to calculate the total driving time, $U$ is the heat transfer coefficient, $S^{k}$ is the surface area of vehicle $k$, and $\Delta T$ is the difference in temperature between the inside and the outside of the vehicle. The heat entering when the door opens is calculated as

$$
H_{s}=\sum_{i \in V} \sum_{j \in V^{\prime}} \sum_{k \in K} x_{i j}^{k} h_{i}
$$

where $h_{i}$ is the heat entering during service time at stop $i$, which is calculated as

$$
h_{i}=\frac{V^{k} c_{p, a}\left(T_{i}^{k}-T_{0}\right)}{3.6 \times 10^{6}},
$$


where $V^{k}$ is the volume of vehicle $k, c_{p, a}$ is the volumetric heat capacity of air, and the factor $3.6 \times 10^{6}$ is used to convert $J$ to $k W h$.

The total fuel used for refrigeration of the load can then be calculated as

$$
f_{r}=\frac{H_{w}+H_{s}}{\eta_{e} \eta_{p} P_{f}}
$$

where $f_{r}$ refers to the fuel used for refrigeration of the load of the vehicle, $\eta_{e}$ is the efficiency by which the chemical energy from the fuel is converted to electricity to drive the refrigeration system, and $\eta_{p}$ is the coefficient of performance, which measures how much thermal energy can be removed with a certain amount of electrical energy. The coefficient of performance is also often referred to as COP (Tassou et al., 2009) but we use $\eta_{p}$ for the formulation.

The emissions related to refrigerated transport are a function of fuel used for motion, fuel used for refrigeration, and refrigerant leakage. Refrigerant leakage emissions can be approximated by multiplying the emissions needed for refrigeration by a given factor. Equation (3.23) shows the calculation of emissions caused by refrigeration of the load and Equation (3.24) gives the total emissions for refrigerated transport:

$$
E_{r}=f_{r} e_{f} e_{r},
$$

where $E_{r}$ are the emissions of refrigerated transport, and $e_{r}$ is the emissions factor that converts emissions caused by fuel use into emissions caused by both fuel use and refrigerant leakage. The total emissions associated with temperature controlled transportation are then

$$
\begin{aligned}
E & =E_{m}+E_{r} \\
& =f_{m} e_{f}+f_{r} e_{f} e_{r} \\
& =\frac{\sum_{i \in V} \sum_{j \in V} \sum_{k \in K} \alpha w_{i j}^{k p} c_{i j}+\sum_{i \in V} \sum_{j \in V} \sum_{k \in K} \beta c_{i j} x_{i j}^{k} v^{2}}{3.6 \times 10^{6} P_{f} \eta_{m}} e_{f} \\
& +\frac{\sum_{i \in V^{\prime}} \sum_{j \in V^{\prime}} \sum_{k \in K} x_{i j}^{k} c_{i j} U S^{k} \Delta T}{3.6 \times 10^{6} v_{i j} \eta_{e} \eta_{p} P_{f}} e_{f} e_{r} \\
& +\frac{\sum_{i \in V} \sum_{j \in V^{\prime}} \sum_{k \in K} x_{i j}^{k} h_{i}}{\eta_{e} \eta_{p} P_{f}} e_{f} e_{r} .
\end{aligned}
$$

\section{Cost calculation}

The total transportation cost can be calculated as follows by adding wage cost and fuel cost:

$$
C=\sum_{i \in V} \sum_{j \in V} \sum_{k \in K} \frac{c_{w} c_{i j} x_{i j}^{k}}{v_{i j}}+\sum_{i \in V^{\prime}} \sum_{j \in V} \sum_{k \in K} c_{w} x_{i j}^{k} s_{i}+\left(f_{m}+f_{r}\right) c_{f},
$$

where $C$ refers to the costs, $c_{w}$ is the driver wage per time unit, $c_{f}$ is the unit fuel cost, $c_{w}$ is the unit wage cost, and $s_{i}$ is the unloading time at node $i$. Note that fuel is used both 
for driving ( $f_{m}$, see Equation 3.17) and for temperature control ( $f_{r}$, see Equation 3.22).

\section{Formulation of objectives and constraints}

For the quality driven VRP model, we consider the three objective functions of minimising product decay (3.26), minimising $\mathrm{CO}_{2}$ emissions (3.27), minimising cost (3.28), and minimising the maximum decay (3.29), which have been defined in terms of the model parameters and variables in Equations (3.3), (3.13), (3.24), and (3.25):

Minimise $D$

Minimise $E$

Minimise $C$

Minimise $Z$

subject to

$$
\begin{aligned}
& \sum_{i \in V^{\prime}} \sum_{k \in K} x_{0 i}^{k} \leq K \\
& \sum_{j \in V} \sum_{k \in K} w_{j i}^{k p}-\sum_{j \in V} \sum_{k \in K} w_{i j}^{k p}=q_{i}^{p} \quad i \in V^{\prime}, p \in P \\
& w_{i 0}^{k p}=0 \quad i \in V^{\prime}, k \in K, p \in P \\
& w_{i j}^{k p} \leq\left(L^{k}-q_{i}^{p}\right) x_{i j}^{k} i \in V, j \in V, k \in K, p \in P \\
& w_{0 j}^{k p} \leq L^{k} x_{0 j}^{k} \quad j \in V, k \in K, p \in P \\
& w_{i j}^{k p} \geq\left(q_{j}^{p}\right) x_{i j}^{k} \quad i \in V, j \in V, k \in K, p \in P \\
& \sum_{i \in V} \sum_{j \in V^{\prime}} \frac{c_{i j} x_{i j}^{k}}{v_{i j}}+\sum_{i \in V} \sum_{j \in V^{\prime}} x_{i j}^{k} s_{i} \leq d \quad k \in K \\
& \sum_{i \in V} \sum_{j \in V} \sum_{k \in K} x_{i j}^{k}=0 \quad i=j \\
& \sum_{i \in V} x_{i j}^{k}=\sum_{j \in V} x_{j i}^{k} \quad j \in V, k \in K \\
& \sum_{i \in V} \sum_{k \in K} x_{i 0}^{k}=\sum_{i \in V^{\prime}} \sum_{k \in K} x_{0 i}^{k}
\end{aligned}
$$




$$
\begin{aligned}
& \sum_{j \in V} x_{0 j}^{k} \leq 1 \quad k \in K \\
& Q_{0 i}^{k p}=Q_{0}^{p} x_{0 i}^{k} \quad i \in V^{\prime}, p \in P, k \in K \\
& \sum_{j \in V} Q_{j i}^{k p}-\sum_{j \in V} Q_{i j}^{k p}=D_{i}^{k p}+\sum_{j \in V} D_{i j}^{k p} i \in V^{\prime}, p \in P, k \in K \\
& Q_{i j}^{k p} \leq Q_{0} x_{i j}^{k p} \quad i \in V^{\prime}, j \in I, p \in P, k \in K \\
& Q_{i j}^{k p} \geq Q_{\min } \quad i \in V, j \in I, p \in P, k \in K \\
& x_{i j}^{k} \in\{0,1\} \quad i \in V, j \in V, k \in K \\
& \sum_{i \in V} \sum_{j \in V} \sum_{k \in K} x_{i j}^{k}=0 \quad i=j \\
& s c_{i j}^{k} \leq \sum_{p \in P} w_{i j}^{k p} t_{c}\left(\frac{T_{i}-T_{0}}{T_{a}-T_{0}}+0.001\right)+M z_{i j}^{k} \quad i \in V^{\prime}, j \in V, k \in K, \\
& -s c_{i j}^{k} \leq-\sum_{p \in P} w_{i j}^{k p} t_{c}\left(\frac{T_{i}-T_{0}}{T_{a}-T_{0}}+0.001\right)+M z_{i j}^{k} i \in V^{\prime}, j \in V, k \in K, \\
& s c_{i j}^{k} \leq \frac{x_{i j}^{k} c_{i j}}{v_{i j}}+M z_{i j}^{k} \\
& i \in V^{\prime}, j \in V, k \in K \text {, } \\
& -s c_{i j}^{k} \leq-\frac{x_{i j}^{k} c_{i j}}{v_{i j}}+M z_{i j}^{k} \\
& z_{i j}^{k} \in\{0,1\} \\
& i \in V^{\prime}, j \in V, k \in K \\
& i \in V^{\prime}, j \in V, k \in K \text {, }
\end{aligned}
$$

where $\mathrm{M}$ is a very large number. Constraints (3.30) specify that no more than the maximum number of vehicles available $(K)$ leave the CDC. Constraints (3.31) are balance constraints; after a node is visited, the load of the vehicle diminishes with the demand delivered to that node. Constraints (3.32) force the vehicle to return to the CDC empty. Constraints (3.33) - (3.35) set boundaries on the minimum and maximum weight transported over an edge and connect decision variables $x_{i j}^{k}$ and $w_{i j}^{k p}$ such that the emission-minimising objective function can remain linear. Constraints (3.36) limit the maximum working time per driver. Constraints (3.37) forbid routes between the same location. Constraints (3.38) enforce that if a node is entered by a vehicle, it should leave from the same node. Constraints (3.39) state that the number of vehicles leaving the CDC should be equal to the number returning. Constraints (3.40) force the model to use a new vehicle when a new route from the CDC is started. Constraints (3.41) set the initial quality level. Constraints (3.42) are the quality balance constraints. Constraints (3.43) ensure that the quality cannot exceed the initial quality. Constraints (3.44) define the minimum quality level. Constraints (3.45) are binary constraints. Constraints (3.46) do not allow the model to suggest routes between the same location. Constraints (3.47) - (3.51) define the cooling time. They ensure that the cooling time is either equal to a function of the load, the cooling speed, and the difference in temperature of the vehicle, and the goal temperature, or 
equal to the total arc crossing time.

\subsection{Computational results and discussion}

In the computational experiments, we first test the effect of using different objective functions (minimising cost, emissions, total decay, and maximum decay) on a number of key performance indicators: decay, emissions, cost, distance, travelling time and computation time. In terms of quality decay, we measure total decay, which is the sum of the decays of the different products at the different locations; average quality, which is the quality that the partners receive on average; and minimum quality, which is the lowest quality received by (one of the) partners. We also show how quality changes along a delivery route. Then, in our sensitivity analyses we test the effects of different parameters in the QDVRP on the KPIs when decay is minimised. We test the effects of using a quality threshold level, different unloading rates, outside temperature, driving speed, cooling rate, and different optimal product temperatures.

The model was coded and solved exactly using Fico Xpress Mosel version 8.0 on a PC with Intel Core i5 processor $(2.6 \mathrm{GHz})$ and eight $\mathrm{GB}$ of memory.

\section{Data and assumptions}

We base our study on demand data of three types of vegetables for the different supermarket chains. Of those products, the optimal temperatures, and parameters that describe the degradation rate for different quality attributes have been studied (Tsironiet al., 2017). The distances between the cooperative's central distribution centre (CDC) and the distribution centres (DC) of each supermarket chain are given in Table 3.2.

Table 3.2: Distances (in km) between the DCs of the supermarket chains (denoted by 1-9) and the $\mathrm{CDC}$ (denoted by 0 ).

\begin{tabular}{r|rrrrrrrrrr} 
& 0 & 1 & 2 & 3 & 4 & 5 & 6 & 7 & 8 & 9 \\
\hline 0 & 0 & 91 & 6 & 134 & 82 & 117 & 74 & 192 & 129 & 120 \\
1 & 91 & 0 & 91 & 75 & 134 & 43 & 20 & 155 & 59 & 122 \\
2 & 6 & 91 & 0 & 134 & 84 & 117 & 75 & 194 & 129 & 123 \\
3 & 134 & 75 & 134 & 0 & 168 & 46 & 94 & 90 & 29 & 118 \\
4 & 82 & 134 & 84 & 168 & 0 & 158 & 140 & 180 & 169 & 90 \\
5 & 117 & 43 & 117 & 46 & 158 & 0 & 59 & 117 & 18 & 128 \\
6 & 74 & 20 & 75 & 94 & 140 & 59 & 0 & 174 & 77 & 128 \\
7 & 192 & 155 & 194 & 90 & 180 & 117 & 174 & 0 & 99 & 110 \\
8 & 129 & 59 & 129 & 29 & 169 & 18 & 77 & 99 & 0 & 137 \\
9 & 120 & 122 & 123 & 118 & 90 & 128 & 128 & 110 & 137 & 0
\end{tabular}

The kinetic parameters of the three products and demands of the three products for the DCs can be found in Table 3.3. The following assumptions are also made for the base case: For all products, the optimal temperature is $275 \mathrm{~K}\left(2^{\circ} \mathrm{C}\right)$, and the vehicle's goal temperature is $275 \mathrm{~K}\left(2^{\circ} \mathrm{C}\right)$ as well. The ambient temperature is $293 \mathrm{~K}$. The unloading time is 0.8 seconds per $\mathrm{kg}$ of load. The cooling time is 0.4 seconds per $\mathrm{kg}$ of load in the truck. Since the products in the case are fresh cut vegetables it was assumed that the order reaction 
of the Arrhenius equation was a zero order reaction (Tsironi et al., 2017). There is a threevehicle fleet available. Each vehicle has an empty weight of $10000 \mathrm{~kg}$ and a capacity of $30000 \mathrm{~kg}$.

Table 3.3: Kinetic parameters and demand $(\mathrm{kg})$ of each product for all DCs.

\begin{tabular}{cccc}
\hline Kinetic parameter & Product 1 & Product 2 & Product 3 \\
\hline$k_{0}\left(\mathrm{~s}^{-1}\right)$ & $3.08 \times 10^{-6}$ & $3.84 \times 10^{-6}$ & $1.96 \times 10^{-6}$ \\
$E_{a}(\mathrm{~J} / \mathrm{mol})$ & 77900 & 76304 & 88521 \\
\hline $\mathrm{DC}$ & & & \\
\hline 1 & 432 & 432 & 216 \\
2 & 2160 & 648 & 648 \\
3 & 648 & 216 & 432 \\
4 & 792 & 403 & 432 \\
5 & 1080 & 432 & 648 \\
6 & 979 & 86 & 259 \\
7 & 864 & 432 & 432 \\
8 & 432 & 108 & 216 \\
9 & 144 & 86 & 101 \\
\hline
\end{tabular}

\section{Base case: comparing different objective functions}

We run the model with different objectives and different numbers of vehicles to better understand how food quality changes in a cooperative route. We also show the model results for individual routing to compare cooperative and individual route planning. The results are summarised in Table 3.4. In this Table, the total decay is the (dimensionless) sum of the decays of all products of all customers, while the minimum and the average decay are percentages of the original quality that the customers receive.

Table 3.4: KPIs resulting from minimising the different objectives for different numbers of vehicles.

\begin{tabular}{|c|c|c|c|c|c|c|c|c|c|c|}
\hline \multirow[b]{3}{*}{ Available vehicles } & \multirow{3}{*}{$\begin{array}{c}\text { Indiv. } \\
7\end{array}$} & \multicolumn{9}{|c|}{ Minimisation objective } \\
\hline & & \multicolumn{3}{|c|}{ Cost } & \multicolumn{3}{|c|}{ Total and maximum decay } & \multicolumn{3}{|c|}{ Emission } \\
\hline & & 2 & 3 & 4 & 2 & 3 & 4 & 2 & 3 & 4 \\
\hline Total decay & 4.7 & 4.53 & 4.47 & 4.47 & 4.53 & 4.47 & 4.43 & 4.53 & 4.53 & 4.53 \\
\hline - Minimum quality (\%) & 85 & 58 & 58 & 58 & 58 & 58 & 71 & 58 & 58 & 58 \\
\hline - Average quality (\%) & 92 & 81 & 83 & 83 & 81 & 83 & 88 & 81 & 81 & 81 \\
\hline Emission $\left(\mathrm{kg} \mathrm{CO}_{2}\right)$ & 1695 & 886 & 888 & 888 & 886 & 888 & 1074 & 886 & 886 & 886 \\
\hline Cost $(€)$ & 1074 & 570 & 562 & 562 & 570 & 562 & 676 & 570 & 570 & 570 \\
\hline Distance $(\mathrm{km})$ & 1393 & 639 & 644 & 644 & 639 & 644 & 808 & 639 & 639 & 639 \\
\hline Travel time (h) & 13.4 & 10.0 & 8.7 & 8.7 & 10.0 & 8.7 & 9.4 & 10.0 & 10.0 & 10.0 \\
\hline Used vehicles & 7 & 2 & 3 & 3 & 2 & 3 & 4 & 2 & 2 & 2 \\
\hline Computation time (s) & 3 & 7 & 30 & 574 & 27 & 480 & 1231 & 11 & 24 & 24 \\
\hline
\end{tabular}

Table 3.4 shows that cooperation results in lower total decay, emission, costs, distance, travel time, and vehicle use. The average quality that is delivered to the customer is higher, as well as the minimum quality. However, the average and the minimum quality that is received in the non-cooperative scenario are higher. Table 3.4 also shows the results for different objective functions for different fleet sizes. It shows that the solution is the same for all objectives when two vehicles are used. For one vehicle, the model becomes infeasible because the maximum driving time constraint is violated. For three vehicles, decay and cost minimisation result in the same solution, which is not that different from the emission-minimising solution in terms of decay, costs and emissions. When using 
four vehicles, the cost and emission solution are the same as in the three-vehicle cost and emission minimising scenarios, but the decay-minimisation is quite different with a lower decay, and significantly higher emissions and costs. Allowing for more than four vehicles with the different objectives gives the same results as the four-vehicle scenarios for those objectives. Minimising total decay and minimising the maximum decay results in the same solutions. In our case, minimising the maximum decay does not help to increase the quality that the partners receive.

\section{Sensitivity analyses}

In this section we test the effect of a threshold value for a minimum quality level, unloading rate, outside temperature, driving speed, cooling rate, and optimal temperature on the results of the quality decay minimisation model.

\section{Threshold for minimum quality level}

Since total decay minimisation can result in some customers receiving a rather low product quality (reflected in the minimum product quality in Table 3.4), we test the effect of quality thresholds (Table 3.5).

Table 3.5: Effect of quality threshold on quality delivered based on order in a route, and on the other KPIs.

\begin{tabular}{cccccccccccc}
\hline $\begin{array}{c}\text { Thres- } \\
\text { hold (\%) }\end{array}$ & Vehicles & 1 & 2 & 3 & 4 & 5 & decay & Emissions & Cost & $\begin{array}{c}\text { Travelling } \\
\text { time }\end{array}$ & $\begin{array}{c}\text { Computa- } \\
\text { tion time }\end{array}$ \\
\hline 80 & 4 & 91 & 85 & & & & 4.6 & 1268 & 801 & 12.4 & 120 \\
$\leq 71$ & 4 & 91 & 87 & 75 & & & 4.4 & 1074 & 676 & 9.4 & 132 \\
0 & 3 & 92 & 89 & 80 & 73 & 64 & 4.5 & 888 & 562 & 8.7 & 480 \\
0 & 2 & 91 & 85 & 80 & 73 & 64 & 4.5 & 886 & 570 & 10.0 & 27 \\
\hline
\end{tabular}

Table 3.5 shows the average product quality received based on the order at which a customer is visited, as well as the total decay, emissions, cost, travelling time and computation time. Since we expected quality thresholds to result in shorter routes, we allow for four vehicles instead of three. Allowing for more than four vehicles gives the same results as allowing for four vehicles, so those results are not shown. As a comparison, also the three- and two-vehicle results without threshold level are shown. Testing a threshold of $85 \%$ or higher results in infeasible solutions since at some individual routes there is already $15 \%$ quality loss for some products, and a threshold level of lower than $71 \%$ results in the same solution as having no threshold since the minimum quality level when there is no threshold is $71 \%$. Table 3.5 shows that a higher quality threshold results shorter routes; in the $80 \%$ threshold route, only two customers are visited per route. Also, it shows that using four vehicles instead of three results in a lower total quality decay as it allows for shorter routes. However, the highest threshold (80\%) causes an increase in decay, as well as in cost and emissions.

\section{Unloading rate}

In the model, an unloading rate is assumed based on communication with practise. We test different unloading rate to test how influential it is in terms of quality decay (Table 3.6). 
Table 3.6: Effect unloading rate on KPIs and routes.

\begin{tabular}{lccc}
\hline Rate $(\mathrm{s} / \mathrm{kg})$ & 0.4 & 0.8 & 1.2 \\
\hline Total decay $(-)$ & 2.7 & 4.5 & 6.3 \\
- Minimum quality (\%) & 74 & 58 & 61 \\
- Average quality (\%) & 90 & 83 & 80 \\
Emission $\left(\mathrm{kg} \mathrm{CO}_{2}\right)$ & 888 & 888 & 1071 \\
Cost $(€)$ & 553 & 562 & 696 \\
Distance $(\mathrm{km})$ & 644 & 644 & 803 \\
Travelling time $(\mathrm{h})$ & 7.7 & 8.7 & 12.2 \\
Computation time $(\mathrm{s})$ & 120 & 480 & 542 \\
\hline
\end{tabular}

Table 3.6 shows that the door opening time is higher and the temperature increase at a stop is higher when the unloading rate is lower. When unloading happens twice as fast (which can be caused by automation in loading/unloading), total decay can reduce with $40 \%$ and the minimum received quality and the average quality increase with 16 and 7 percentage points, respectively. When increasing unloading rate, costs and emissions stay the same, or improve.

\section{Outside temperature}

Table 3.7 the effect of the outside temperature on the KPIs when decay is minimised.

Table 3.7: Effect outside temperature on KPIs and routes.

\begin{tabular}{lcccc}
\hline Outside temperature $\left({ }^{\circ} \mathrm{C}\right)$ & 2 & 10 & 20 & 30 \\
\hline Total decay $(-)$ & 1.0 & 1.8 & 4.5 & 11.9 \\
- Minimum quality $(\%)$ & 92 & 84 & 58 & 18 \\
- Average quality $(\%)$ & 96 & 93 & 83 & 62 \\
Emission $\left(\mathrm{kg} \mathrm{CO}_{2}\right)$ & 729 & 801 & 888 & 1164 \\
Cost $(€)$ & 501 & 524 & 562 & 724 \\
Distance $(\mathrm{km})$ & 639 & 644 & 644 & 803 \\
Travelling time $(\mathrm{h})$ & 10.0 & 8.7 & 8.7 & 10.7 \\
Computation time $(\mathrm{s})$ & 3 & 27 & 42 & 883 \\
\hline
\end{tabular}

As the results imply, the outside temperature is an important factor in quality decay. When we combine this with the effect of unloading rate, we can see that in warmer countries, it is especially important to unload quickly. Also, at a temperature of $30^{\circ} \mathrm{Celsius,} \mathrm{the}$ minimum quality reaches a level $(18 \%)$ that will probably be unacceptable for most customers.

\section{Driving speed}

We varied the speed matrix to test different average driving speeds (Table 3.8). The base case (scenario 1.0) has an average driving speed of $55 \mathrm{~km} / \mathrm{h}$, and the other scenarios (denoted 0.6-1.4) define by which number the speed matrix is multiplied to obtain the new speed matrix. The base case speed matrix can be found in the Appendix (Table A.5).

Table 3.8 shows that a higher driving speed can reduce total quality decay, while for emissions and cost, the intermediate driving speeds give better results. However, in terms of minimum and average decay level, a lower driving speed (up to 0.8 times the base case speed matrix) results in better solutions. This is because the lower driving speeds result in two two-destination routes and one three-destination route, while the faster scenarios ( 0.9 times the base case speed and up) result in two one-destination routes and one five- 
Table 3.8: Effect of driving speed on KPIs and routes.

\begin{tabular}{|c|c|c|c|c|c|c|c|c|c|}
\hline Scenario & 0.6 & 0.7 & 0.8 & 0.9 & 1.0 & 1.1 & 1.2 & 1.3 & 1.4 \\
\hline Route 1 & $(0,2,4,0)$ & \multicolumn{2}{|c|}{$(0,2,4,0)$} & \multicolumn{6}{|c|}{$(0,2,0)$} \\
\hline Route 2 & $(0,3,7,0)$ & \multirow{2}{*}{\multicolumn{2}{|c|}{$\begin{array}{c}(0,5,3,7,0) \\
(0,6,1,0)\end{array}$}} & \multirow{2}{*}{\multicolumn{6}{|c|}{$(0,6,1,5,3,7,0)$}} \\
\hline Route 3 & $(0,6,1,5,0)$ & & & & & & & & \\
\hline Total decay (-) & 5.0 & 4.8 & 4.7 & 4.6 & 4.5 & 4.4 & 4.3 & 4.3 & 4.3 \\
\hline - Minimum quality (\%) & 74 & 69 & 70 & 57 & 58 & 59 & 59 & 60 & 60 \\
\hline - Average quality (\%) & 86 & 85 & 86 & 83 & 83 & 83 & 84 & 84 & 84 \\
\hline Emission $\left(\mathrm{kg} \mathrm{CO}_{2}\right)$ & 1024 & 1003 & 1020 & 867 & 888 & 913 & 941 & 972 & 1006 \\
\hline Cost $(€)$ & 744 & 694 & 684 & 561 & 562 & 567 & 575 & 585 & 598 \\
\hline Distance $(\mathrm{km})$ & 844 & 803 & 803 & 644 & 644 & 644 & 644 & 644 & 644 \\
\hline Travelling time $(\mathrm{h})$ & 16.3 & 14.0 & 12.6 & 9.5 & 8.7 & 8.1 & 7.6 & 7.2 & 6.9 \\
\hline Computation time (s) & 198 & 120 & 39 & 325 & 480 & 685 & 976 & 577 & 1314 \\
\hline
\end{tabular}

destination route. In terms of emissions and costs, a speed close to the base case results in the best performance.

\section{Cooling rate}

Different cooling rates were tested and the results are presented in Table 3.9.

Table 3.9: Effect of cooling rate on KPIs and routes.

\begin{tabular}{lcccc}
\hline Scenario & Fast & Base & Medium & Slow \\
Cooling rate $(\mathrm{s} / \mathrm{kg})$ & 0.2 & 0.4 & 0.6 & 0.8 \\
\hline Total decay $(-)$ & 4.4 & 4.5 & 4.6 & 4.6 \\
- Minimum quality (\%) & 73 & 58 & 57 & 56 \\
- Average quality (\%) & 83 & 83 & 83 & 83 \\
Emission $(\mathrm{kg} \mathrm{CO})$ & 1073 & 888 & 888 & 888 \\
Cost $(€)$ & 684 & 562 & 562 & 562 \\
Distance $(\mathrm{km})$ & 802 & 644 & 644 & 644 \\
Travel time $(\mathrm{h})$ & 10.5 & 8.7 & 8.7 & 8.7 \\
Computation time $(\mathrm{s})$ & 28 & 480 & 1699 & 7656 \\
\hline
\end{tabular}

Table 3.9 shows that only a very fast cooling rate changes the optimal route and consequently, the cost and emissions. However, the effect of cooling rate is much smaller compared with the effect of unloading rate. As a consequence of the different route at the fastest cooling rate, the minimum quality level does improve significantly.

\section{Optimal temperature}

So far, we have assumed that all products had the same optimal temperature $\left(275 \mathrm{~K}, 2^{\circ} \mathrm{C}\right)$. Here, we test the effect of different optimal temperatures, while minimising quality decay. Table 3.10 shows that a higher average optimal temperature significantly decreases decay. This implies that considering quality decay is very important for temperaturesensitive products with a low optimal temperature, especially if they are transported in a warm environment. In the scenario with three different optimal temperatures, the products are transported at the average optimal temperature. This causes product 1 to be transported above its optimal temperature, but still, the average and minimum decay are better than in the scenario where all products need a low temperature. 
Table 3.10: Effect optimal product temperature on KPIs and routes. $T_{0}$ is the goal temperature in the vehicle. $T_{1}-T_{3}$ are the optimal temperatures of product 1 to 3 .

\begin{tabular}{lccc}
\hline Optimal temperature & Low & Medium & Variable \\
\hline$T_{0}(\mathrm{~K})$ & 275 & 280 & 280 \\
$T_{1}(\mathrm{~K})$ & 275 & 280 & 275 \\
$T_{2}(\mathrm{~K})$ & 275 & 280 & 280 \\
$T_{3}(\mathrm{~K})$ & 275 & 280 & 285 \\
\hline Total decay $(-)$ & 4.5 & 1.4 & 2.3 \\
- Minimum quality (\%) & 58 & 76 & 62 \\
- Average quality $(\%)$ & 83 & 90 & 89 \\
Emission $\left(\mathrm{kg} \mathrm{CO}_{2}\right)$ & 888 & 845 & 845 \\
Cost $(€)$ & 562 & 543 & 543 \\
Distance $(\mathrm{km})$ & 644 & 644 & 644 \\
Travelling time $(\mathrm{h})$ & 8.7 & 8.7 & 8.7 \\
Computation time $(\mathrm{s})$ & 480 & 405 & 326 \\
\hline
\end{tabular}

\subsection{Conclusions}

We have introduced, modelled and solved the Quality Driven Vehicle Routing Problem (QDVRP), which is an extension of the traditional VRP, that explicitly considers the quality aspects of food and perishable transportation. Our model was applied to a cooperative setting to study the effects of multi-stop routing on food quality, but also on cost and emissions. Compared with cost and emission minimisation, decay minimisation resulted in using more vehicles and driving sorter routes. When driving longer routes, the product quality arriving at locations visited later in the route is lower. This difference in quality level could be corrected by setting a threshold quality level for all locations. This however results in higher costs and emissions. In our case study, we also tried to minimise the maximum decay to reduce the quality difference between locations but this did not result in other solutions compared with total decay minimisation. However, in a setting with more alternative routes, this approach may yield different solutions. Our sensitivity analyses showed that faster unloading (e.g., by automation in loading/unloading process) or faster cooling (i.e. equipping the vehicles with better cooling engines) can reduce total quality decay. Also, technical improvements to prevent heat from entering the vehicles when unloading could reduce decay. The outside temperature is also very influential on the decay rate. However, this is hard to influence, but one could choose to transport food products very early in the morning or at night to benefit from a lower outside temperature, and to avoid traffic jams. Also, faster driving can decrease total decay. However, in our case study, a lower speed resulted in a higher minimum and average quality. Moreover, an intermediate speed results in better costs and emissions.

The QDVRP model can be applied to gain insights into quality decay on a multi-stop route. Also, possible cooperative partners can use this model to measure the impacts of food logistics cooperation on costs, emissions and quality decay. Moreover, the model can be used to test the effect of technological improvements to reduce quality decay during road transportation. 
3.5. Conclusions 


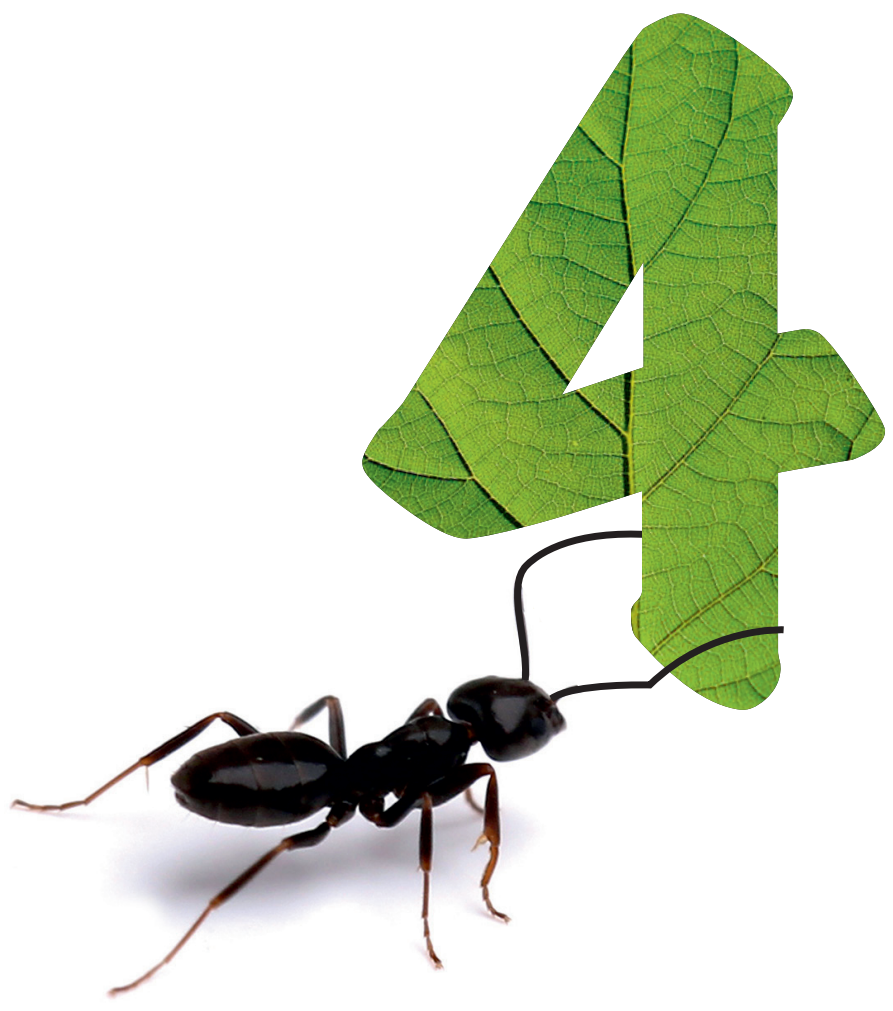




\section{Chapter 4}

\section{Quantifying the environmental and economic benefits of cooperation: A case study in temperature-controlled food logistics}

Inefficient road transportation causes unnecessary costs and polluting emissions. This problem is even more severe in refrigerated transportation, in which temperature control is used to guarantee the quality of the products. Organising logistics cooperatively can help decrease both the environmental and the economic impacts. In Joint Route Planning (JRP) cooperation, suppliers and customers jointly optimise routing decisions so that cost and emissions are minimised. Vendor Managed Inventory (VMI) cooperation extends JRP cooperation by optimising routing and inventory planning decisions simultaneously. However, in addition to their economic advantages, VMI and JRP may also yield environmental benefits. To test this assertion, we perform a case study on cooperation between a number of supermarket chains in the Netherlands. The data of this case study are analysed to quantify both the economic and environmental benefits of implementing cooperation via JRP and VMI, using vehicle routing and an inventory routing models. We found that JRP cooperation can substantially reduce cost and emissions compared with uncooperative routing. In addition, VMI cooperation can further reduce cost and emissions, but minimising cost and minimising emissions no longer result in the same solution and there is a trade-off to be made.

This chapter is based on: Stellingwerf, H.M., Laporte, G. Cruijssen, F.C.A.M., Kanellopoulos, A., Bloemhof, J.M. (2018). Quantifying the environmental and economic benefits of cooperation: A case study in temperaturecontrolled food logistics. Transportation Research part D: Transport and Environment 65: 178-193. 


\subsection{Introduction}

Road transportation generates significant costs for firms that deliver and collect products, and it is also a source of negative environmental impacts such as $\mathrm{CO}_{2}$ emissions (Palmer, 2007). The transportation of frozen and fresh food, pharmaceuticals and flowers cause additional polluting emissions because more fuel is required to control the vehicle temperature during transportation (Hariga et al., 2017). Moreover, extra emissions are associated with the leakage of refrigeration fluid (Stellingwerfet al., 2018a). Also during storage, temperature control can result in additional costs and emissions (Bozorgi et al., 2014; Bazan et al., 2015). For fresh food, temperature control is needed to keep high product quality and to ensure a longer shelf life. A literature review has shown that there are very few papers that have considered sustainability in the transportation of perishable products (Vrat et al., 2018). Cooperative logistics has been suggested as a way of reducing carbon emissions and of improving sustainability in supply chains (Ramanathan et al., 2014; Vanovermeire et al., 2014; McKinnon, 2016; Chen et al., 2017), especially in food supply chains (Bloemhofet al., 2015). Apart from saving cost and reducing emissions, cooperative logistics also offers other advantages such as an improved utilisation rate of vehicles and an increased transportation capacity (Cruijssen et al., 2007a).

This research focuses on two types of logistics cooperation: Joint Route Planning (JRP) and Vendor-Managed Inventory (VMI). In JRP, firms aim to save cost by organising transportation more efficiently, either by cooperating or by outsourcing part of their logistics planning to a logistics service provider. JRP research has traditionally focused on economic indicators (Cruijssen et al., 2007b). To enable JRP, cooperative firms need to share information on their transportation needs. In VMI, the vendor manages its own inventory as well as the inventory of its customers by taking decisions on how much and at which frequency to replenish; the vendor is responsible for keeping the stock level of the customer within agreed limits (Nagarajan and Sošić, 2008; Bazan et al., 2015). VMI is often described as a win-win situation: vendors save on distribution cost as they are able to combine and coordinate shipments for different customers, and customers save by not allocating efforts in controlling and managing inventories (Coelhoet al., 2013). Often, VMI is seen as a cooperative relation between a supplier and its customers. However, it is also possible for multiple suppliers to cooperatively implement VMI from a joint warehouse. Another possibility is that a group of customers hire a logistics service provider to store and distribute the products for them. Thus, VMI is a logistics concept that can enable different forms of cooperation. It requires a higher level of information sharing compared with JRP since in addition to information on transportation needs, information on the inventories also needs to be shared. Research has shown that firms see the reduction of emissions as one of the reasons to implement VMI (Makaci et al., 2017). However, to our knowledge there is no research quantifying the environmental effects of implementing cooperation via VMI. Indeed, most research on VMI based-cooperation focuses on the effects on economic indicators such as cost or profit (Borade and Sweeney, 2015; Chakraborty et al., 2015; Cai et al., 2017).

The goal of this Chapter is to quantify the environmental and economic benefits of imple- 
menting cooperation via VMI and JRP for a fresh food supply chain in the Netherlands. In order to quantify both the environmental and economic effects of implementing VMI and JRP, we need a model that not only considers distance, but also vehicle load, the order of deliveries and vehicle speed (Bektaş and Laporte, 2011; Zachariadis et al., 2015). It is also necessary to consider the effects of using temperature-controlled transportation which can have significant effects on both cost and emissions. Therefore we apply the temperature-controlled Load Dependent Vehicle Routing Problem model (Stellingwerf et al., 2018a) to compute optimal routes in a JRP setting. For the VMI, we also need to consider inventory decisions for a supplier and multiple clients, delivery frequency, and delivery quantities. Therefore, the green Inventory Routing Problem (IRP) is an appropriate modelling tool for estimating the environmental and economic benefits of cooperative VMI (Cheng et al., 2017). It is an inventory routing model that can be used both for minimising emissions and for minimising costs by jointly optimising inventory and routing decisions. Here we extend the green IRP to include the effects of temperature control and to test the effect of IRP decisions on average product age.

We apply these models to study a case of cooperative fresh food logistics between seven supermarket chains in the Netherlands, a form of buyer cooperation. Both cost and emissions are quantified as indicators of economic and environmental benefits. JRP and VMI are compared with each other, as well as with the current situation in which transportation is planned individually by each supermarket chain. The remainder of the Chapter is structured as follows. In Section 4.2 we introduce the case study, in Section 4.3 we mathematically formulate the problem, in Section 4.4 we show and discuss the results, and in Section 4.5 we conclude this Chapter and suggest future research directions.

\subsection{Case study: cooperative VMI to reduce cost and emissions in Dutch supermarket chains}

In the Netherlands, several smaller supermarket chains have opted to cooperate by forming a buying organisation. By jointly purchasing their products, they can negotiate a lower unit price. The supermarket chains currently hire different logistics service providers to pick up their orders from the buying organisation's central distribution centre (CDC) and bring them to their own distribution centre (DC). From their DC, they distribute the products to their supermarket outlets. These supermarket chains consider the possibility of intensifying their cooperation to further reduce cost and emissions, not only by using their alliance as a buying organisation, but also by cooperatively managing their routing and inventory decisions.

Each supermarket chain owns between 30 and 300 supermarket outlets. We focus on the cooperation between the CDC and the DCs. The supermarket chains use three main temperature categories for their foods: (i) frozen, (ii) chilled, and (iii) ambient. Frozen products should be kept at around $-18^{\circ} \mathrm{C}$, and generally have a long shelf life (a few months). Chilled products should be kept at around $2^{\circ} \mathrm{C}$, and are generally characterised by a short shelf life (a few days). Ambient temperature products do not need temperature control, and can either have a long shelf life, in the case of dry products like biscuits, or a medium shelf life (several days to several weeks), in the case of fresh products such as fruits and 
vegetables.

Currently, the supermarket chains arrange their logistics individually, but they are considering to cooperate. There exist two options to this end: Joint Route Planning (JRP) and Vendor Managed Inventory (VMI), and they would like to measure the effects of these forms of cooperation on their economic and environmental performance. They would also like to know whether the effects vary for the different temperature categories. Moreover, they wish to assess the effects of the different scenarios on other key performance indicators such as emissions, driving distance, cost, total travelling time, and average product age upon leaving the DC. We use a green IRP model to quantify these key performance indicators in order to analyse cooperation via JRP and VMI, as well as individual routing (the current situation).

\subsection{Formal problem description and mathematical formulation}

The problem under consideration is NP-hard since it encompasses a Vehicle Routing Problem (VRP) which is known to be NP-hard. Here, we provide the formulation of a green IRP, which is used to model cooperation via VMI. After the model formulation, we add constraints so that cooperation via JRP and individual routing (the current situation) can be modelled as well. A summary of all notations and values used is given in the Appendix (Tables A.1-A.4).

Let $G=(V, A)$ be a graph in which $V=\{0,1, \ldots, n\}$ is the set of nodes. The CDC is located at vertex $0, V \backslash\{0\}$ is the set of DCs, and $A=\{(i, j): i, j \in V, i \neq j\}$ is the set of arcs. With every arc $(i, j)$ is associated a non-negative distance $c_{i j}$. We define $t$ as an index for the set of time periods $\{1, \ldots, T\}$, where $T$ is the length of the planning horizon. The demand at each DC $i$ in each period $t$ is given by $q_{i}^{t}$, and $C_{i}$ is the capacity of DC $i$. There exists a set of identical vehicles $K=\{1, \ldots, k\}$ of capacity $L$ with a curb (empty) weight of $L_{0}(\mathrm{~kg})$. We denote by $v_{i j}$ the speed driven on $\operatorname{arc}(i, j)$.

The following decision variables are used:

- $x_{i j}^{k t}$ is a binary variable equal to 1 if and only if vehicle $k$ drives from node $i$ to node $j$ in period $t$;

- $w_{i j}^{k t}$ is the total weight carried, including the vehicle weight, from node $i$ to node $j$ by vehicle $k$ in period $t$;

- $y_{i}^{k t}$ is the weight of the products delivered to DC $i$ by vehicle $k$ in period $t$

- $I_{i}^{t}$ is the inventory level of DC $i$ in time period $t$.

\section{Fuel and emissions calculation in ambient transportation}

Bektaş and Laporte (2011) have shown that the fuel use in ambient transport is linearly related to the motive power requirement. The latter depends on the weight carried, the slope of the road, the distance travelled and the vehicle speed. The motive power $P_{i j}$ on $\operatorname{arc}(i, j)$ can be approximated as

$$
P_{i j}=\alpha_{i j} w_{i j}^{k t} c_{i j}+\beta v_{i j}^{2} c_{i j},
$$


where $\alpha_{i j}$ is the arc-specific constant, and $\beta$ is the vehicle-specific constant. Equations (4.2) and (4.3) show how these constants are calculated:

$$
\alpha_{i j}=a+g \sin \theta_{i j}+g C_{r} \cos \theta_{i j},
$$

where $a$ is the acceleration of the vehicle $\left(\mathrm{m} / \mathrm{s}^{2}\right), g$ is the gravitation constant $\left(\mathrm{m} / \mathrm{s}^{2}\right), \theta_{i j}$ refers to the average slope on arc $i j$ (degrees), $C_{r}$ is the rolling resistance (dimensionless). The vehicle-specific constant is calculated as

$$
\beta=0.5 C_{d} A \rho,
$$

where $C_{d}$ is the drag coefficient (dimensionless), $A$ is the frontal area of the vehicle $\left(\mathrm{m}^{2}\right)$, and $\rho$ is the air density $\left(\mathrm{kg} / \mathrm{m}^{3}\right)$.

In (4.4) fuel use for ambient transport $\left(f_{m}\right)$ is calculated by summing up the power requirements for all routes and converting those into fuel use. This is achieved by dividing the power by $3.6 \times 10^{6}$ to convert Joule (J) to kilowatt-hour ( $\mathrm{kWh}$ ), by the chemical to motive energy conversion efficiency $\left(\eta_{m}\right)$, and by the energy content of the fuel $\left(P_{f}\right)$ :

$$
f_{m}=\left(\sum_{i \in V} \sum_{j \in V} \sum_{k \in K} \sum_{t \in T} \alpha w_{i j}^{k t} c_{i j}+\sum_{i \in V} \sum_{j \in V} \sum_{k \in K} \sum_{t \in T} \beta c_{i j} x_{i j}^{k t} v_{i j}^{2}\right) \frac{1}{3.6 \times 10^{6} P_{f} \eta_{m}} .
$$

The emissions from ambient transport are linearly related to fuel use:

$$
E_{m}=f_{m} e_{f}
$$

where $e_{f}$ is the emissions factor which converts fuel use into $\mathrm{CO}_{2}$ emissions $(\mathrm{kg} / \mathrm{L})$, and $E_{m}$ are the $\mathrm{CO}_{2}$ emissions of ambient transport, i.e. the motive emissions $(\mathrm{kg})$.

\section{Fuel and emissions calculation in refrigerated transportation}

Stellingwerfetal. (2018a) have approximated the cost, the fuel consumption and the emissions of refrigerated transport, so that the impact of temperature controlled transport can be estimated in route optimisation models. In refrigerated transport, fuel is used both for motive power and for keeping the temperature of the load at the right level. The energy used for temperature control depends on the heat that enters through the vehicle wall during driving time, and on the heat that enters the vehicle when the door opens. The heat entering through the wall $(\mathrm{kWh})$ is calculated as

$$
H_{w}=\frac{\sum_{i \in V^{\prime}} \sum_{j \in V^{\prime}} \sum_{k \in k} \sum_{t \in T} x_{i j}^{k t} c_{i j} U S^{k} \Delta T}{3.6 \times 10^{6} v_{i j}},
$$

where $x_{i j}^{k t} c_{i j} / v_{i j}$ is used to calculate the total driving time (s), $U$ is the heat transfer coefficient $\left(\mathrm{W} / \mathrm{m}^{2} / \mathrm{K}\right), S^{k}$ is the surface area of vehicle $k\left(\mathrm{~m}^{2}\right)$, and $\Delta T$ is the difference in temperature between the inside and the outside of the vehicle $(\mathrm{K})$. The heat entering when the door opens $(\mathrm{kWh})$ is calculated as

$$
H_{s}=\sum_{i \in V} \sum_{j \in V^{\prime}} \sum_{k \in K} \sum_{t \in T} x_{i j}^{k t} h_{i}
$$


where $h_{i}$ is the heat entering during service time at stop $i(\mathrm{kWh})$.

The total fuel used for refrigeration of the load can then be calculated as

$$
f_{r}=\frac{H_{w}+H_{s}}{\eta_{e} \eta_{p} P_{f}}
$$

where $f_{r}$ refers to the fuel used for refrigeration of the load of the vehicle, $\eta_{e}$ is the efficiency by which the chemical energy from the fuel is converted to electricity to drive the refrigeration system (dimensionless), and $\eta_{p}$ is the coefficient of performance, which measures how much thermal energy can be removed with a certain amount of electrical energy (dimensionless). The coefficient of performance is also often referred to as COP (Tassou et al., 2009) but we use $\eta_{p}$ for the formulation.

The emissions related to refrigerated transport are a function of fuel used for motion, fuel used for refrigeration, and refrigerant leakage. Refrigerant leakage emissions can be approximated by multiplying the emissions needed for refrigeration by a given factor. Equation (4.9) shows the calculation of emissions caused by refrigeration of the load and Equation (4.10) gives the total emissions for refrigerated transport:

$$
E_{r}=f_{r} e_{f} e_{r},
$$

where $E_{r}$ are the emissions of refrigerated transport $\left(\mathrm{kg} \mathrm{CO}_{2}\right)$, and $e_{r}$ is the emissions factor that converts emissions caused by fuel use into emissions caused by both fuel use and refrigerant leakage $(\mathrm{kg} / \mathrm{kg})$. The total emissions associated with temperature controlled transportation are then

$$
\begin{aligned}
E & =E_{m}+E_{r} \\
& =f_{m} e_{f}+f_{r} e_{f} e_{r} \\
& =\frac{\sum_{i \in V} \sum_{j \in V} \sum_{k \in K} \sum_{t \in T} \alpha w_{i j}^{k t} c_{i j}+\sum_{i \in V} \sum_{j \in V} \sum_{k \in K} \sum_{t \in T} \beta c_{i j} x_{i j}^{k t} v^{2}}{3.6 \times 10^{6} P_{f} \eta_{m}} e_{f} \\
& +\frac{\sum_{i \in V^{\prime}} \sum_{j \in V^{\prime}} \sum_{k \in k} \sum_{t \in T} x_{i j}^{k t} c_{i j} U S^{k} \Delta T}{3.6 \times 10^{6} v_{i j} \eta_{e} \eta_{p} P_{f}} e_{f} e_{r} \\
& +\frac{\sum_{i \in V} \sum_{j \in V^{\prime}} \sum_{k \in K} \sum_{t \in T} x_{i j}^{k t} h_{i}}{\eta_{e} \eta_{p} P_{f}} e_{f} e_{r} .
\end{aligned}
$$

\section{Cost calculation}

The transportation cost can be calculated as follows by adding wage cost and fuel cost:

$$
C_{t}=\sum_{i \in V} \sum_{j \in V} \sum_{m \in M} \sum_{t \in T} \frac{c_{w} c_{i j} x_{i j}^{k t}}{v_{i j}}+\sum_{i \in V^{\prime}} \sum_{j \in V} \sum_{k \in K} \sum_{t \in T} c_{w} x_{i j}^{k t} s+\left(f_{m}+f_{r}\right) c_{f},
$$

where $C_{t}$ refers to the transportation cost $(€), c_{w}$ is the driver wage per time unit $(€ / \mathrm{s})$, $c_{f}$ is the unit fuel cost $(€ / \mathrm{L})$, and $c_{w}$ is the unit wage cost $(€ / \mathrm{s})$. Note that fuel is used both 
for driving ( $f_{m}$, see Equation 4.4) and for temperature control ( $f_{r}$, see Equation 4.8).

The inventory cost is calculated as

$$
C_{I}=\sum_{i \in V} \sum_{t \in T} I_{i}^{t} c_{I}
$$

where $C_{I}$ refers to the inventory cost $(€), I_{i}^{t}$ refers to the inventory level at location $i$ in period $t(\mathrm{~kg}), c_{I}$ are the unit inventory cost $(€ / \mathrm{kg} /$ day).

The total cost $C$ is then

$$
\begin{aligned}
C & =C_{t}+C_{I} \\
& =\sum_{i \in V} \sum_{j \in V} \sum_{k \in K} \sum_{t \in T} \frac{c_{w} c_{i j} x_{i j}^{k t}}{v_{i j}}+\sum_{i \in V^{\prime}} \sum_{j \in V} \sum_{k \in K} \sum_{t \in T} c_{w} x_{i j}^{k t} s+\left(f_{m}+f_{r}\right) c_{f} \\
& +\sum_{i \in V} \sum_{t \in T} I_{i}^{t} c_{I} \\
& =\sum_{i \in V} \sum_{j \in V} \sum_{m \in M} \sum_{t \in T} \frac{c_{w} c_{i j} x_{i j}^{k t}}{v_{i j}}+\sum_{i \in V^{\prime}} \sum_{j \in V} \sum_{k \in K} \sum_{t \in T} c_{w} x_{i j}^{k t} s \\
& +\frac{\sum_{i \in V} \sum_{j \in V} \sum_{k \in K} \sum_{t \in T} \alpha w_{i j}^{k t} c_{i j} \sum_{i \in V} \sum_{j \in V} \sum_{k \in K} \sum_{t \in T} \beta c_{i j} x_{i j}^{k t} v_{i j}^{2}}{3.6 \times 10^{6} P_{f} \eta_{m}} \\
& +\frac{\sum_{i \in V^{\prime}} \sum_{j \in V^{\prime}} \sum_{k \in k} \sum_{t \in T} x_{i j}^{k t} c_{i j} U S^{k} \Delta T}{3.6 \times 10^{6} v_{i j} \eta_{e} \eta_{p} P_{f}} c_{f} \\
& +\frac{\sum_{i \in V} \sum_{j \in V^{\prime}} \sum_{k \in K} \sum_{t \in T} x_{i j}^{k t} h_{i}}{\eta_{e} \eta_{p} P_{f}} c_{f} \\
& +\sum_{i \in V} \sum_{t \in T} I_{i}^{t} c_{I} .
\end{aligned}
$$

\section{A green IRP formulation for temperature-controlled food transportation}

For the green IRP model (Cheng et al., 2017), we consider the two objective functions of minimising $\mathrm{CO}_{2}$ emissions (4.14) and minimising cost (4.15), which have been defined in terms of the model parameters and variables in Equations (4.10) and (4.13):

Minimize $E$

Minimize $C$ 
subject to

$$
\begin{aligned}
& I_{0}^{t}=I_{0}^{t-1}-\sum_{i \in V^{\prime}} \sum_{k \in K} y_{i}^{k t} \quad t \in\{2, \ldots, T\} \\
& I_{0}^{1}=\sum_{i \in V^{\prime}} \sum_{t \in T} q_{i}^{t} \\
& I_{i}^{t}=I_{i}^{t-1}+\sum_{k \in K} y_{i}^{k t}-q_{i}^{t} \quad i \in V^{\prime}, t \in T \\
& I_{i}^{1}=0 \quad i \in V^{\prime} \\
& I_{i}^{t} \geq 0 \quad i \in V^{\prime}, t \in T \\
& I_{i}^{t} \leq C_{i} \quad i \in V^{\prime}, t \in T \\
& \sum_{k \in K} y_{i}^{k t} \leq C_{i}-I_{i}^{t-1} \quad i \in V^{\prime}, t \in\{2, \ldots, T\} \\
& q_{i}^{k t} \leq C_{i} \sum_{j \in V} x_{i j}^{k t} \quad i \in V^{\prime}, k \in K, t \in T \\
& w_{i j}^{k t} \leq\left(L+L_{0}\right) x_{i j}^{k t} \quad i \in V, j \in V, k \in K, t \in T \\
& w_{i j}^{k t} \geq L_{0} x_{i j}^{k t} \quad i \in V, j \in V, k \in K, t \in T \\
& \sum_{j \in V} x_{i j}^{k t} \leq n \sum_{j \in V^{\prime}} x_{0 j}^{k t} \quad i \in V, k \in K, t \in T \\
& \sum_{k \in K} \sum_{i \in V} x_{i j}^{k t} \leq 1 \quad j \in V^{\prime}, t \in T \\
& \sum_{j \in V} x_{i j}^{k t}=\sum_{j \in V} x_{j i}^{k t} \quad i \in V, k \in K, t \in T \\
& \sum_{i \in V \mid i \neq j} w_{i j}^{k t}-\sum_{i \in V \mid i \neq j} w_{j i}^{k t}=y_{i}^{k t} \quad j \in V^{\prime}, k \in K, t \in T \\
& \sum_{j \in V^{\prime}} x_{0 j}^{k t} \leq 1 \quad k \in K, t \in T \\
& x_{i j}^{k t}+x_{j i}^{k t} \leq 1 \quad i \in V^{\prime}, j \in V^{\prime}, k \in K, t \in T \\
& x_{i i}^{k t}=0 \quad i \in V, k \in K, t \in T \\
& w_{i j}^{k t} \geq 0 \quad i \in V, j \in V, k \in K, t \in T \\
& y_{i}^{k t} \geq 0 \quad i \in V^{\prime}, k \in K, t \in T \\
& I_{i}^{t} \geq 0 \quad i \in V, t \in T \\
& x_{i j}^{k t} \in\{0,1\} \quad i \in V, j \in V, k \in K, t \in T .
\end{aligned}
$$

Constraints (4.16) - (4.17) define the inventory at the CDC, while constraints (4.18) - (4.21) define the inventories at the DCs. Constraints (4.22) are the maximum level inventory policy constraints. Constraints (4.23) ensure that no delivery can be made at DC $i$ in period $t$ when there is no vehicle visiting that node in that period. Constraints (4.24) and 
(4.25) are the vehicle capacity constraints. Constraints (4.26) restrict the number of vehicles used per day. Constraints (4.27) restrict the number of visits to each DC to at most one per day. Constraints (4.28) are the flow conservation equations. Constraints (4.29) eliminate sub tours. Constraints (4.30) ensure that at most one vehicle per day visits each location. Constraints (4.31) state that a vehicle leaves from the same DC as the one it entered. Constraints (4.32) eliminate impossible arcs. Constraints (4.33) - (4.35) are nonnegativity constraints, while constraints (4.36) are binary constraints.

In the IRP model (Equations (4.14) - (4.36)), the routing, the frequency and quantity of delivery are optimised. In the VRP model, only the routing is optimised. To model cooperative JRP, the IRP model had to be transformed into a VRP model. This is achieved by adding constraints (4.37) which limit the quantity delivered to a DC on each day to the DC demand on that day:

$$
\sum_{k \in K} y_{i}^{k t} \leq q_{i}^{t} \quad i \in V^{\prime}, t \in T
$$

To quantify the effect of individual routing, we extend the VRP model by adding constraint (4.38) that allows the model to at maximum bring the demand of one destination from the $C D C$, such that it chooses individual daily routes:

$$
w_{0 j}^{k t} \leq L_{0}+q_{j}^{k t} \quad j \in V^{\prime}, k \in K, t \in T .
$$

\section{Average product age calculation}

For fresh products, the time required to move from the CDC to the supermarkets is an indicator of freshness and quality. We refer to this time as product age. The shorter the total shelf life, the more important it is to deliver the products to the supermarket as quickly as possible. We can calculate the average product age by using the demand data and the model outputs on the quantity delivered and on the inventory on all time periods. To this end, we assume that all DCs apply a FIFO (first in, first out) policy, and that all products arrive at the DC with an age of one day. We take the outputs of the IRP, VRP and individual routing models defined by (4.14) - (4.38) and use them as an input for the average product age calculation.

We first define $r$ as an index for the set of ages $\{1, \ldots, R\}$. As an input for the age calculation model we use the demand $q_{i}^{t}$, the quantity delivered $y_{i}^{t}$ and the inventory $I_{i}^{t}$, all for each DC $i \in V^{\prime}$ and in each time period. As decision variables, we consider $q_{i r}^{t}$ as the demand fulfilled from DC $i$ by a product of age $r$ in period $t, y_{i r}^{t}$ as the quantity delivered to DC $i$ of age $r$ in period $t$, and $I_{i r}^{t}$ as the inventory at DC $i$ of age $r$ in period $t$. The following objective is then set to enforce the FIFO policy:

$$
\text { Minimize } \sum_{i \in V^{\prime}} \sum_{t \in T} \sum_{r \in R} r I_{i r}^{t}
$$


subject to

$$
\begin{array}{rlrl}
\sum_{r \in R} q_{i r}^{t} & =q_{i}^{t} & & i \in V^{\prime}, t \in T \\
\sum_{r \in R} I_{i r}^{t}=I_{i}^{t} & & i \in V^{\prime}, t \in T \\
\sum_{r \in 1 . .1} y_{i r}^{t}=y_{i}^{t} & i \in V^{\prime}, t \in T \\
I_{i r}^{t} & =I_{i, r-1}^{t-1}-q_{i r}^{t}+y_{i r}^{t} & i \in V^{\prime}, a \in\{2, \ldots, A\}, t \in\{2, \ldots, T\} .
\end{array}
$$

Constraints (4.40) mean that for each DC in each period, the sum of the product demands over all ages is equal to the total demand. Constraints (4.41) ensure that for each DC in each period, the sum of the inventory of all different ages equals the total inventory. Constraints (4.42) state that for each DC in each period, the age of each incoming product is one, and that the sum off all incoming products of age one equals the total of incoming products. Constraints (4.43) describe how the inventory ages over time.

The average age $(\bar{r})$ of the products leaving to the supermarkets is then calculated as

$$
\bar{r}=\frac{\sum_{i \in V^{\prime}} \sum_{t \in T} \sum_{r \in R} r q_{i r}^{t}}{\sum_{i \in V^{\prime}} \sum_{t \in T} q_{i}^{t}} .
$$

\subsection{Computational results and discussion}

In our Computational experiments, we compare three scenarios: (i) no cooperation (imitating the current situation in which routing is planned individually), (ii) JRP cooperation where the supermarket chains use a common logistics service provider to reduce transportation cost but do not share sensitive inventory information, and (iii) VMI cooperation where the chains decide to share inventory information to optimise delivery frequency by the logistics service provider. These scenarios are illustrated in Figure 4.1.

For the no cooperation scenario, we assume that there is a separate route back and forth between the the CDC and each DC. For the JRP scenario, we assume that the supermarkets inform the CDC of their demand for the next day and that the CDC makes an optimised route plan and hires a logistics service provider to fulfil all demands. In the VMI scenario, the supermarkets share information on a daily basis on their inventory, and on the minimum and maximum level of the products. The daily demand of each location is known, and the CDC makes an optimised delivery plan based on those data.

We calculate a number of key performance indicators: distance, cost, emissions, travelling time, average product age, and computation time. Note that the travelling time includes the time spent unloading at the DCs. We focus on comparing the results of the cooperative JRP and VMI scenarios with those of the current non-cooperative situation. We also identify trade-offs between cost and emissions, and between cost and product age. We then perform sensitivity analyses to investigate the effect of parameter changes. 


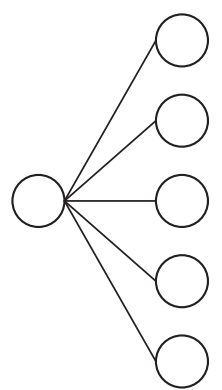

Individual routing

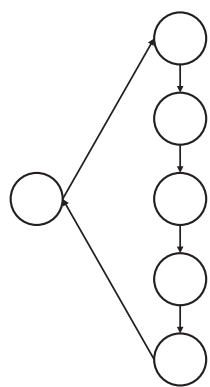

Joint route planning

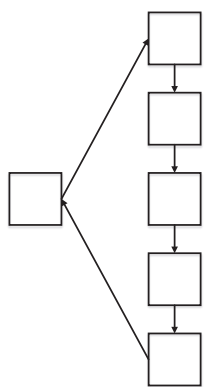

Vendormanaged inventory

Figure 4.7: Supply chain scenarios. The circles indicate locations, and the blocks indicate locations for which the inventory is known.

Finally, we compare the impacts of foods held at different temperatures on the key performance indicators, as well as the effect of each scenario on the average age of the products. The model was coded and solved exactly using CPLEX 12.7 (which uses a branchand-bound algorithm) on a PC with Intel Core is processor $(2.6 \mathrm{GHz})$ and eight $\mathrm{CB}$ of memory.

\section{Data and assumptions}

The CDC provided us with data on a week of fresh food orders placed by the DCs, on which we ran our experiments. Table 4.1 gives the distance matrix, and Table 4.2 provides the demand matrix based on that study. The distances between the locations are estimated based on the road distances between the postal codes of the locations using the ESRI ArcCIS software. Note that Sunday is left out of the demand table, because the DCs and the $\mathrm{CDC}$ are closed on that day and hence do not place orders. Starting inventories are assumed to be 0 in the DCs and to be equal to the sum of the weekly demand in the CDC. We assume a maximum level $(\mathrm{ML})$ inventory policy, in which any quantity of products can be delivered as long as the DC's maximum level is not exceeded. Concerning the routing, each day the vehicles start and end at the CDC. Given that our case study is in the Netherlands, we assume that the slopes on all routes are zero, which means that the arc-specific constant $\alpha_{i j}(4.2)$ is equal to $0.0981 \mathrm{~m} / \mathrm{s}^{2}$ for all arcs. In countries where there are more significant slopes, specific slope data could be incorporated in the experiments. In our case study, deliveries are generally made between 4 am and 7 am and DCs are located outside of densely populated areas to avoid traffic jams, which is why we apply a fixed speed of $72 \mathrm{~km} / \mathrm{h}(20 \mathrm{~m} / \mathrm{s})$ on all arcs.

We assume that the inventory cost is equal to one tenth of the sales price at the final outlet, and decreases by a factor 10 with each level it moves up in the supply chain (Soysal 
et al., 2015). We assume that all products are sold at $1.00 € / \mathrm{kg}$, hence at the supermarkets the inventory cost are $0.10 € / \mathrm{kg} / \mathrm{day}$, at the DC they are $0.01 € / \mathrm{kg} / \mathrm{day}$, and at the CDC they are $0.001 € / \mathrm{kg} /$ day. We use a homogeneous fleet with medium duty vehicles that have a curb weight of $5500 \mathrm{~kg}$, and a capacity of $12500 \mathrm{~kg}$ (Koçet al., 2016). The capacities are expressed in terms of weight and not in terms of volume because temperature-controlled food products are generally relatively heavy and are therefore likely to reach their maximum weight before they reach their maximum volume. The three-vehicle fleet can cover the weekly demand.

All DCs have a capacity equal to their demand for the week. Finally, we assume that the ambient temperature is $20^{\circ} \mathrm{C}(293 \mathrm{~K})$, and that the temperature in the vehicles transporting the fresh food needs to be kept at $2^{\circ} \mathrm{C}(275 \mathrm{~K})$. All parameter values are summarised in the Appendix (Table A.3).

Table 4.1: Distances (in km) between the locations: CDC (0), DCs (1-7).

\begin{tabular}{r|rrrrrrrr} 
& 0 & 1 & 2 & 3 & 4 & 5 & 6 & 7 \\
\hline 0 & 0 & 91 & 6 & 134 & 82 & 117 & 74 & 192 \\
1 & 91 & 0 & 91 & 75 & 134 & 43 & 20 & 155 \\
2 & 6 & 91 & 0 & 134 & 84 & 117 & 75 & 194 \\
3 & 134 & 75 & 134 & 0 & 168 & 46 & 94 & 90 \\
4 & 82 & 134 & 84 & 168 & 0 & 158 & 140 & 180 \\
5 & 117 & 43 & 117 & 46 & 158 & 0 & 59 & 117 \\
6 & 74 & 20 & 75 & 94 & 140 & 59 & 0 & 174 \\
7 & 192 & 155 & 194 & 90 & 180 & 117 & 174 & 0
\end{tabular}

Table 4.2: Demand in kg at each DC.

\begin{tabular}{r|rrrrrr} 
DC & Monday & Tuesday & Wednesday & Thursday & Friday & Saturday \\
\hline 1 & 540 & 540 & 450 & 450 & 810 & 0 \\
2 & 1890 & 1680 & 1260 & 2760 & 2220 & 1170 \\
3 & 270 & 630 & 360 & 540 & 1440 & 0 \\
4 & 0 & 1122 & 0 & 1248 & 0 & 0 \\
5 & 1260 & 810 & 1350 & 1260 & 540 & 1080 \\
6 & 1170 & 1524 & 1170 & 1608 & 2412 & 0 \\
7 & 0 & 720 & 360 & 720 & 360 & 450
\end{tabular}

\section{Comparison between current situation, JRP, and VMI}

We compared the current situation, where routing and inventory are planned individually with JRP and VMI. For JRP and VMI, we compared the effects of cooperatively minimising cost as well as of cooperatively minimising emissions. Results are shown in Table 4.3. This table shows that JRP can yield significant savings in costs and emissions, as well as in distance and in travelling time. By implementing VMI, these savings become even more substantial.

Table 4.3 would thus suggest that when partners choose from JRP or VMI, they would generally go for VMI. However, VMI requires them to share inventory information, which is often considered as sensitive information. Implementing VMI therefore requires a certain level of trust. The results also show that for JRP the solutions for minimising cost 
Table 4.3: Key performance indicators for different fresh food cooperation scenarios for emissions and cost minimisation.

\begin{tabular}{lrrrrr}
\hline & Current & $\begin{array}{c}\text { JRP } \\
\text { emissions }\end{array}$ & $\begin{array}{c}\text { JRP } \\
\text { cost }\end{array}$ & $\begin{array}{r}\text { VMI } \\
\text { emissions }\end{array}$ & $\begin{array}{r}\text { VMI } \\
\text { cost }\end{array}$ \\
\hline Distance $(\mathrm{km})$ & 6715 & 2789 & 2789 & 808 & 1241 \\
Cost $(€)$ & 2043 & 966 & 966 & 1158 & 692 \\
- Transportation cost $(€)$ & 1920 & 843 & 843 & 270 & 408 \\
- Inventory cost $(€)$ & 123 & 123 & 123 & 888 & 284 \\
CO $_{2}$ emissions $(\mathrm{kg})$ & 3718 & 1631 & 1631 & 522 & 789 \\
Average product age (days) & 1.00 & 1.00 & 1.00 & 3.35 & 1.48 \\
Travelling time (h) & 102 & 47 & 47 & 13 & 22 \\
Computation time $(\mathrm{s})$ & 1 & 89 & 94 & 11 & 176 \\
\hline
\end{tabular}

and for minimising emissions are the same. For VMI, minimising cost and minimising emissions result in a different optimal solution and when implementing VMI, the average product age is higher. It depends on the type of product if this is important: the shorter the total shelf life, the more important it is to move the product through the supply chain quickly.

\section{Trade-off analysis}

Table 4.3 shows that VMI can further reduce emissions and costs compared to JRP. However, when $\mathrm{VMI}$ is implemented, minimising cost results in a different optimal solution than minimising emissions. Also, when minimising emissions in $\mathrm{VMI}$, costs become slightly higher than in JRP. Previous research has suggested that it would be interesting to establish a trade-off between cost and emissions in a Green IRP context (Cheng et al., 2017). To this end, we have calculated trade-offs between cost and emissions (Figure 4.2) using the $\epsilon$-constraint method (Mavrotas, 2009), and we show the trade-off between age and emissions (Figure 4.3). Minimising emissions results in a solution in which as many products as possible are brought together to the DCs in one order to reduce the transportation activity. However, this will generate a high inventory cost, and consequently a high total cost. Note that transportation cost includes the cost for temperature control. In Figure 4.2 we have added a line below which all solutions are better (i.e. lower) in terms of cost and emissions compared to JRP.

Figure 4.3 shows the trade-off between emissions and product age. Note that there is no trade-off between cost and age since minimising cost results in a lower inventory at the DCs and consequently in a lower average age. These trade-offs could cause a potential obstacle to cooperation, since the different partners would need to agree on where they want to be on the trade-off curve and which consequent cooperative routing and inventory strategy they want to follow. However, if they do agree then the savings in terms of cost and emissions are higher than when JRP is implemented.

\section{Sensitivity analyses}

In this section we test the effect of different temperature products, different inventory capacities, different inventory holding cost, vehicle capacity, and demand and distance data. 


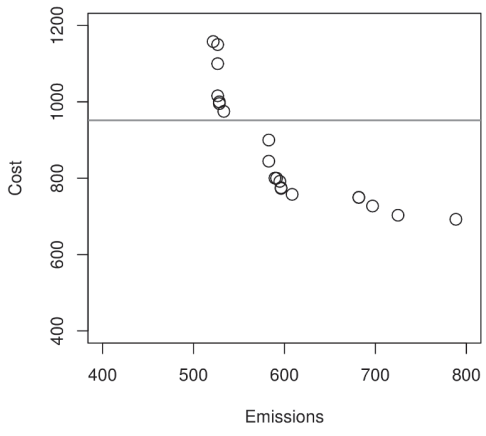

Figure 4.2: Trade-off between $\mathrm{CO}_{2}$ emissions ( $\mathrm{kg}$ ) and cost (€). Below the line, all solutions are better then JRP in terms of both cost and emissions.

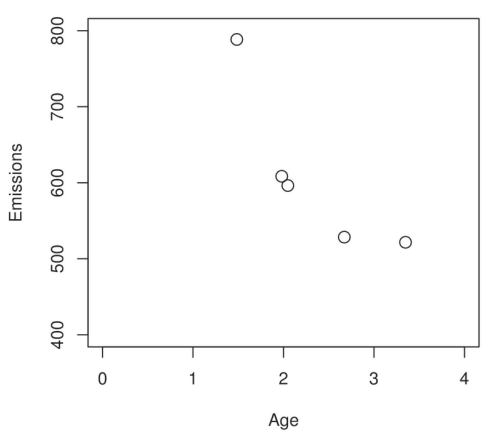

Figure 4.3: Trade-off between average product age upon leaving the DCs (days) and $\mathrm{CO}_{2}$ emissions $(\mathrm{kg})$.

\section{Routing and inventory optimisation: ambient and frozen food}

To test the effect of different temperature products, we changed the product temperature from $2^{\circ} \mathrm{C}$ to $-18^{\circ} \mathrm{C}$ for frozen products and to $20^{\circ} \mathrm{C}$ for ambient products. Table 4.4 shows the distance, cost, emissions, average product age upon leaving the DC and the total travelling time for frozen products, under cost and emissions minimisation, and for VMI, JRP and individual routing. Table 4.5 provides the same statistics for ambient products. For these scenarios, we assumed the same demands and distances as in Tables 4.1 and 4.2 , but we adjusted the temperature of the products. Note that minimising emissions or cost yields the same solution under]RP. When emissions are minimised for the frozen food VMI (Table 4.4), the optimal routing and inventory plan is the same as when emissions are minimised for the ambient food VMI (Table 4.5). However, when cost is minimised, the optimal solutions for frozen and for ambient food are different from each other. For frozen food, routing is relatively more expensive because of the extra fuel needed to provide cooling. Therefore, the cost minimising solution for frozen food VMI results in a shorter distance, and less frequent replenishments, compared with the cost minimising solution for ambient food. Tables 4.4 and 4.5 also show differences in average product age. Since the shelf life of frozen and ambient temperature foods is generally longer than that of fresh food, the differences in average product age are less important as they have less effect on the remaining shelf life. Also, comparing the cost and emissions of the current situation and the cooperation scenarios for ambient and frozen foods, we see that temperature control results in increased cost and emissions, but also that the total saving in cost and emissions is higher in the case of frozen food when JRP or VMI are implemented. 
Table 4.4: Key performance indicators for different frozen food cooperation scenarios for emissions and cost minimisation.

\begin{tabular}{lrrrrr}
\hline & Current & $\begin{array}{c}\text { JRP } \\
\text { emissions }\end{array}$ & $\begin{array}{c}\text { JRP } \\
\text { cost }\end{array}$ & $\begin{array}{r}\text { VMI } \\
\text { emissions }\end{array}$ & $\begin{array}{r}\text { VMI } \\
\text { cost }\end{array}$ \\
\hline Distance $(\mathrm{km})$ & 6715 & 2789 & 2789 & 808 & 1352 \\
Cost $(€)$ & 2214 & 1037 & 1037 & 1623 & 865 \\
- Transportation cost $(€)$ & 2090 & 914 & 914 & 291 & 472 \\
- Inventory cost $(€)$ & 123 & 123 & 123 & 1332 & 393 \\
CO $_{2}$ emissions $(\mathrm{kg})$ & 4110 & 1793 & 1793 & 569 & 926 \\
Average product age (days) & 1.00 & 1.00 & 1.00 & 3.35 & 1.42 \\
Travelling time $(\mathrm{h})$ & 102 & 47 & 47 & 13 & 24 \\
Computation time $(\mathrm{s})$ & 1 & 13 & 15 & 12 & 1560 \\
\hline
\end{tabular}

Table 4.5: Key performance indicators for different ambient food cooperation scenarios for emissions and cost minimisation.

\begin{tabular}{lrrrrr}
\hline & Current & $\begin{array}{c}\text { JRP } \\
\text { emissions }\end{array}$ & $\begin{array}{c}\text { JRP } \\
\text { cost }\end{array}$ & $\begin{array}{c}\text { VMI } \\
\text { emissions }\end{array}$ & $\begin{array}{r}\text { VMI } \\
\text { cost }\end{array}$ \\
\hline Distance $(\mathrm{km})$ & 6715 & 2789 & 2789 & 808 & 1759 \\
Cost $(€)$ & 1890 & 902 & 902 & 1584 & 782 \\
- Transportation cost $(€)$ & 1766 & 779 & 779 & 252 & 555 \\
- Inventory cost $(€)$ & 123 & 123 & 123 & 1332 & 227 \\
$\mathrm{CO}_{2}$ emissions $(\mathrm{kg})$ & 3366 & 1484 & 1484 & 479 & 981 \\
Average product age (days) & 1.00 & 1.00 & 1.00 & 3.35 & 1.17 \\
Travelling time $(\mathrm{h})$ & 102 & 47 & 47 & 13 & 31 \\
Computation time $(\mathrm{s})$ & 1 & 12 & 15 & 9 & 780 \\
\hline
\end{tabular}

\section{Effect of inventory capacities at the DCs}

In the previous calculations, we assumed that the capacity of each DC is equal to the weekly demand. In this section we test the effect of smaller capacities in the DC, both for minimising cost and for minimising emissions. The results are shown in Table 4.6. For the VMI scenario, different inventory capacities were chosen for the supermarkets. For example, a capacity of 0.8 means that for each supermarket chain, the maximum inventory level is set at $80 \%$ of the total weekly demand.

A lower inventory capacity results in a higher transportation cost and in lower inventory cost for both objectives. For the cost minimisation objective, limiting DC capacity does not have a strong effect on the results because the model also minimises inventory cost if there is enough capacity. For emissions minimisation, the results of changing the inventory capacities exhibits more differences: transportation cost increases, inventory cost decreases, emissions increase, and the average product age decreases. When the inventory capacity is limited, the difference between the optimal solutions for emissions minimisation and cost minimisation are closer to each other. The effect of inventory capacity was only tested on VMI scenarios, since in the JRP and the individual routing scenarios, the inventory arrives at the DC and leaves it on the same day, so that inventory capacity is not constraining. 
Table 4.6: Key performance indicators for VMI with different inventory capacities for emissions and cost minimisation.

\begin{tabular}{lrrrrrr}
\hline & \multicolumn{3}{c}{ Emissions minimisation } & \multicolumn{3}{c}{ Cost minimisation } \\
\hline Capacity $($ ratio total demand) & 1 & 0.8 & 0.6 & 1 & 0.8 & 0.6 \\
\hline Distance $(\mathrm{km})$ & 808 & 1189 & 1366 & 1241 & 1454 & 1454 \\
Cost $(€)$ & 1158 & 945 & 823 & 692 & 710 & 728 \\
- Transportation cost $(€)$ & 270 & 383 & 442 & 408 & 468 & 469 \\
- Inventory cost $(€)$ & 888 & 562 & 382 & 284 & 242 & 259 \\
CO $_{2}$ emissions $(\mathrm{kg})$ & 522 & 741 & 854 & 789 & 906 & 906 \\
Average product age (days) & 3.35 & 2.37 & 1.79 & 1.48 & 1.36 & 1.42 \\
Travelling time $(\mathrm{h})$ & 13 & 20 & 23 & 22 & 26 & 26 \\
Computation time $(\mathrm{s})$ & 11 & 113 & 71 & 176 & 299 & 720 \\
\hline
\end{tabular}

\section{Effect of inventory cost}

To test the effect of inventory cost, we reduced and increased it in some runs. In the previous calculations, we assumed an inventory cost of $0.001 € / \mathrm{kg} /$ day for the CDC, and 0.01 $€ / \mathrm{kg} /$ day for the DC (medium inventory cost). For the low inventory cost level, we now assume $0.0005 € / \mathrm{kg} /$ day for the CDC, and $0.005 € / \mathrm{kg} /$ day for the DC. For the high inventory cost level, we now assume $0.0015 € / \mathrm{kg} /$ day for the CDC, and $0.015 € / \mathrm{kg} / \mathrm{day}$ for the DC. We have tested the effect of these cost levels on the cost minimising VMI as well as on the emissions minimising VMI (Table 4.7).

Table 4.7: Key performance indicators for VMI with different inventory cost levels for emissions and cost minimisation.

\begin{tabular}{lrrrrrr}
\hline & \multicolumn{3}{c}{ Emissions minimisation } & \multicolumn{3}{c}{ Cost minimisation } \\
\hline Inventory cost & low & medium & high & low & medium & high \\
\hline Distance $(\mathrm{km})$ & 808 & 808 & 808 & 950 & 1241 & 1759 \\
Cost $(€)$ & 714 & 1158 & 1602 & 536 & 692 & 822 \\
- Transportation cost $(€)$ & 270 & 270 & 270 & 315 & 408 & 555 \\
- Inventory cost $(€)$ & 444 & 888 & 1332 & 221 & 284 & 267 \\
CO $_{2}$ emissions $(\mathrm{kg})$ & 522 & 522 & 522 & 608 & 789 & 1074 \\
Average product age (days) & 3.35 & 3.35 & 3.35 & 1.98 & 1.48 & 1.17 \\
Travelling time $(\mathrm{h})$ & 13 & 13 & 13 & 17 & 22 & 31 \\
Computation time $(\mathrm{s})$ & 10 & 11 & 10 & 2040 & 176 & 1260 \\
\hline
\end{tabular}

Table 4.7 shows that changing the inventory cost does not impact the optimal route and inventory when emissions are minimised, since in our case emissions are only linked to transportation. For the cost minimisation objective, increasing the inventory cost results in a higher delivery frequency, and hence in a higher transportation cost, higher emissions and a lower average product age. Previous research confirms that it is difficult for companies with high inventory cost to reduce their emissions (Cheng et al., 2017).

\section{Effect of vehicle capacity}

For our base case and all other scenarios, we assumed medium-duty vehicles (MDV) with curb weights of 5,500 kg and maximum payloads of $12,500 \mathrm{~kg}$ (Koç et al., 2016). For individual routing, JRP, and VMI, we tested whether it was possible to improve the results by using different vehicle types: light-duty vehicles (LDV, curb weight of 4,500 kg, maximum payload of 7,500 kg) (Koçet al., 2016), and heavy-duty vehicles (HDV, curb weight of 
$10,000 \mathrm{~kg}$, maximum payload of $30,000 \mathrm{~kg}$ ) (Tassou et al., 2009). The results are shown in Table 4.8.

Table 4.8: Effect of vehicle capacity on key performance indicators of different cooperation scenarios for emissions and cost minimisation.

\begin{tabular}{|c|c|c|c|c|c|c|c|c|}
\hline & \multicolumn{3}{|c|}{ Current } & \multicolumn{2}{|c|}{ JRP emissions } & \multicolumn{3}{|c|}{ JRP cost } \\
\hline & small & medium & large & small & medium & large & small & medium \\
\hline Distance $(\mathrm{km})$ & 6715 & 6715 & 6715 & 2801 & 2789 & 2789 & 2801 & 2789 \\
\hline Cost $(€)$ & 1946 & 2043 & 2480 & 928 & 966 & 1148 & 928 & 966 \\
\hline - Transportation cost (€) & 1823 & 1920 & 2357 & 805 & 843 & 1024 & 805 & 843 \\
\hline - Inventory cost $(€)$ & 123 & 123 & 123 & 123 & 123 & 123 & 123 & 123 \\
\hline $\mathrm{CO}_{2}$ emissions & 3533 & 3718 & 4550 & 1558 & 1631 & 1976 & 1558 & 1631 \\
\hline Average product age (days) & 1.00 & 1.00 & 1.00 & 1.00 & 1.00 & 1.00 & 1.00 & 1.00 \\
\hline Travelling time $(\mathrm{h})$ & 102 & 102 & 102 & 47 & 47 & 47 & 47 & 47 \\
\hline Computational time (s) & 1 & 1 & 1 & 14 & 89 & 10 & 13 & 94 \\
\hline
\end{tabular}

Table 4.8: Effect of vehicle capacity on key performance indicators (continued).

\begin{tabular}{lrrrrrrr}
\hline & JRP cost & \multicolumn{1}{l}{ VMl emission } & \multicolumn{3}{c}{ VMI cost } \\
\hline & large & small & medium & large & small & medium & large \\
\hline Distance $(\mathrm{km})$ & 2789 & 1136 & 808 & 549 & 1445 & 1241 & 1007 \\
Cost $(€)$ & 1148 & 1138 & 1158 & 1159 & 702 & 692 & 767 \\
- Transportation cost $(€)$ & 1024 & 352 & 270 & 250 & 435 & 408 & 397 \\
- Inventory cost $(€)$ & 123 & 786 & 888 & 909 & 267 & 284 & 369 \\
CO $_{2}$ emissions & 1976 & 680 & 522 & 481 & 842 & 789 & 766 \\
Average product age (days) & 1.00 & 3.04 & 3.35 & 3.41 & 1.44 & 1.48 & 1.76 \\
Travelling time (h) & 47 & 18 & 13 & 9 & 25 & 22 & 18 \\
Computational time $(\mathrm{s})$ & 14 & 1528 & 11 & 1 & 281 & 176 & 479 \\
\hline
\end{tabular}

For the individual routing scenarios of the ambient products, it is indeed possible to save on cost and emissions ( $4.8 \%$ and $5.0 \%$, respectively) by using a LDV. For the JRP scenarios, using a LDV also resulted in the lowest cost and emissions (a saving of 3.9\% and $4.4 \%$, respectively). For the emission-minimising VMI situation, using smaller vehicle resulted in a cost saving of $1.7 \%$ and in an emissions increase of $30 \%$. For the cost-minimising VMI situation, the MDV resulted in the lowest costs, while emissions were reduced by $2.8 \%$ when a heavy duty vehicle was used.

\section{Effect of demand and distance data}

To test whether the results were not case study specific, we generated several data sets of the same size as the case study problem, based on the Solomon R101 data set (Solomon, 1987), which is a VRP data set. We converted this data set to a seven-destination six-day IRP data set. This was done by using the demand of locations 1 to 7 of the Solomon data set as the demand of day 1 for DC 1 to 7 . Similarly, the demand of locations 8 to 14 was used for day 2 (DC 1 to 7 ), up to the demand of day 36 to 42 for day 6 (DC 1 to 7 ). Five different data sets were then created for the location data. For data set 1, locations 1 to 7 of the Solomon data set were assumed up to locations 29 to 35 for data set 5 . The $x$ and $y$ coordinates from the Solomon-based locations were converted to distance matrices using Euclidean distances. We then multiplied the distances and the demands by factors such that the average distance was the same as the average distance in the case study, and such 
that the average demand was the same as the average demand in the case study. The demand matrix and the five location matrices were run for the following cooperation settings: individual routing, JRP-cost minimisation, JRP-emissions minimisation, VMl-cost minimisation, and VMI-emissions minimisation, resulting in 25 test instances. All constants were kept the same as in the case study. The test instances are summarised in the Appendix (Table A.6). To show the results in a concise way, we averaged the results of the five data sets for objective-cooperation scenario combination (Table 4.9).

Table 4.9: Key performance indicators different Solomon-based test instances (averaged per cooperation setting and objective function).

\begin{tabular}{lrrrrr}
\hline & $\begin{array}{c}\text { Individual } \\
\text { routing }\end{array}$ & $\begin{array}{c}\text { JRP } \\
\text { emission }\end{array}$ & $\begin{array}{c}\text { JRP } \\
\text { cost }\end{array}$ & $\begin{array}{c}\text { VMI } \\
\text { emission }\end{array}$ & $\begin{array}{r}\text { VMI } \\
\text { cost }\end{array}$ \\
\hline Distance $(\mathrm{km})$ & 7931 & 3367 & 3367 & 926 & 1507 \\
Cost $(€)$ & 2397 & 1197 & 1197 & 1199 & 856 \\
- Transportation cost $(€)$ & 2270 & 1070 & 1070 & 325 & 508 \\
- Inventory cost $(€)$ & 127 & 127 & 127 & 873 & 348 \\
CO $_{2}$ emissions $(\mathrm{kg})$ & 4396 & 2069 & 2069 & 621 & 981 \\
Average product age (days) & 1.00 & 1.00 & 1.00 & 3.30 & 1.68 \\
Travelling time $(\mathrm{h})$ & 121 & 57 & 57 & 15 & 25 \\
Computation time $(\mathrm{s})$ & 1 & 87 & 97 & 265 & 557 \\
\hline
\end{tabular}

Table 4.9 shows that the results based on the Solomon R101 data set are very comparable to those in Table 4.3. There are some difference in the absolute values but the relative savings of moving from individual routing to JRP to VMI (cost and emissions minimisation) are very similar. Also, like in the case study, JRP yields the same optimal solution for minimising costs and minimising emissions, while there is a trade-off to be made in VMI.

\subsection{Conclusions and future work}

This research has illustrated that logistics cooperation can yield substantial monetary and environmental benefits, especially in temperature-controlled supply chains for fresh and frozen food. Specifically, we found that cooperation can reduce cost and emissions but also driving distance and travelling time. We have tested the effects of cooperation using VMI and JRP, and we have compared these two policies with individual route planning. We found that VMI cooperation is the most beneficial but it also requires information sharing between multiple actors. On the other hand, JRP cooperation results in lower but still substantial benefits compared with VMI. In JRP, the same solution results in minimised cost and emissions. In VMI, however, there exists a clear trade-off between minimising cost and minimising emissions. Therefore, in order to implement VMI, the cooperative partners should make a decision on which plan is preferable, based on how important they value cost and emissions savings. We also quantified the effect of different types of cooperation on average product age, as an indicator for food quality and we found that the use of VMI can help in establishing a trade-off between product age and emissions.

Our case study was conducted for seven DCs and one CDC. Cooperating and agreeing with seven partners can be challenging, which is why most other studies on cooperative benefits describe situations with only two or three partners (Cruijssen et al., 2010; Vanovermeire et al., 2014; Vanovermeire and Sörensen, 2014; Defryn et al., 2016). However, in our 
case study there already exists some form of cooperation between the partners, so that extending the cooperation is expected to be less problematic than starting a cooperation from scratch. Still, research has shown that the allocation of benefits can be an important obstacle to overcome in order to ensure a successful cooperation (Cruijssen et al., 2007c). Significant research has focused on how to divide the gains in such a way that they reflect each partner's contribution to the cost savings (Frisk et al., 2010). We believe that a future research direction could be to find a benefit allocation that is fair to all partners and also reflects their contribution to savings in costs and emissions.

Another research extension would be to consider the effect of temperature control on inventory, and the inclusion of different temperature ranges (Bozorgi, 2016). We did not consider this issue since it is very dependent on the source of the electricity used. The quantification of the effect associated with different electricity sources could, however, be an interesting future research question. Another promising development could be to investigate whether cooperation at a multi-echelon level can further increase the cooperative savings, and to what extent. Also, future research on logistics cooperation and on the resulting food quality could benefit from the use of a temperature-dependent quality indicator (Akkerman et al., 2010). Data collection coupled with time-temperature indicators could be of value in this type of research (Van Der Vorst et al., 2009). Indeed, James et al. (2006) have shown that opening the vehicle door and consequent temporarily raising the temperature of the load does not only have an effect on the amount of energy needed for cooling, but also on food quality. 


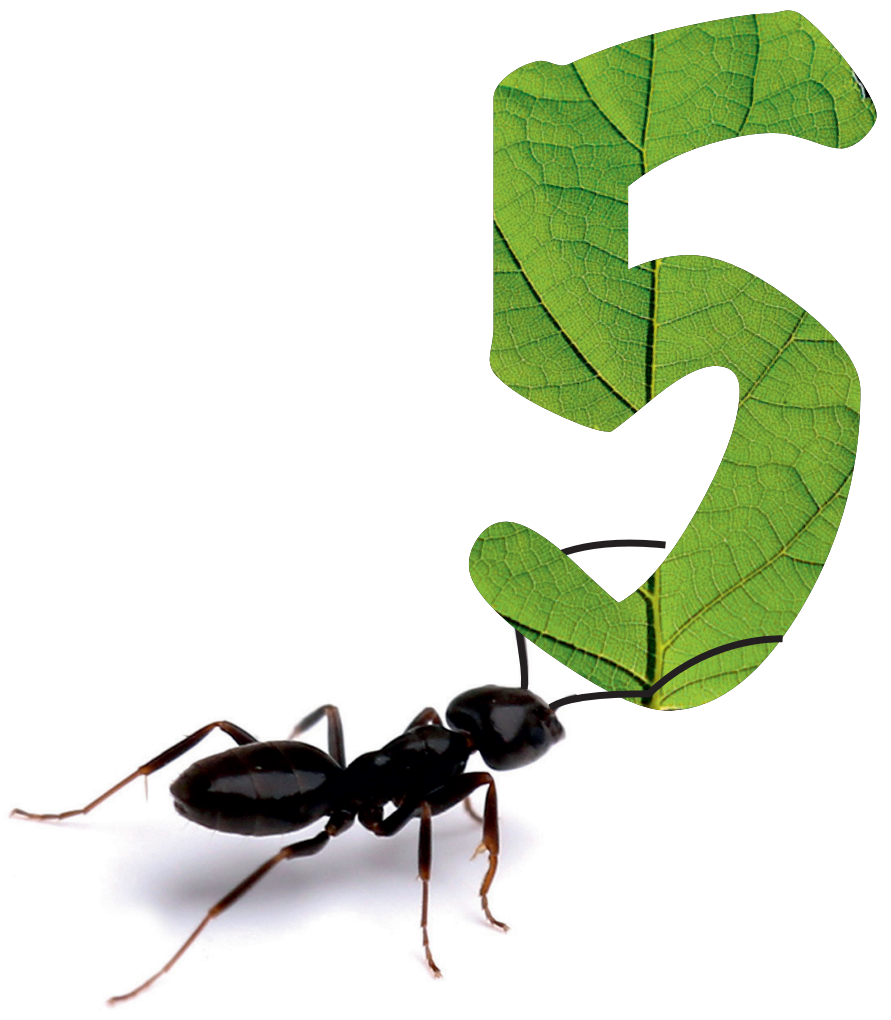




\section{Chapter 5}

\section{Fair gain allocation in eco-efficient vendor-managed inventory cooperation}

Transportation is not always organised efficiently, which causes unnecessary costs and $\mathrm{CO}_{2}$ emissions. Vendor-managed inventory (VMI) has been suggested as a form of cooperation that can reduce economic and environmental impacts of transportation and consequently improve supply chain eco-efficiency. Establishing viable forms of VMI cooperation requires a fair distribution of the cooperation's economic benefits. Cooperative game theory research is used to fairly allocate benefits. However, environmental contributions of partners have often been ignored in the benefit allocation. In this chapter, the Shapley value is used to share the monetary gains in a way that reflects the partners' contributions to cost and emissions savings. The method is applied to evaluate the allocation of economic and environmental benefits of vendor-managed inventory between cooperating supermarket chains in the Netherlands. The findings show that there is a set of eco-efficient solutions resulting in lower costs and emissions compared to the current situation. For each of the eco-efficient solutions, the relative importance of saving costs and of saving emissions was quantified, and based on the importance weights, a cost allocation was found. For all partners that contribute to saving both cost and emissions, this approach results in cost savings, and therefore, the approach can be considered fair and it helps to stimulate long-term eco-efficient forms of cooperation.

This chapter is based on: Stellingwerf, H.M., Kanellopoulos, A., Cruijssen, F.C.A.M., Bloemhof, J.M. (2019). Gain allocation in eco-efficient vendor-managed inventory cooperation, Journal ofCleaner Production, accepted for publication. 


\subsection{Introduction}

Public awareness about global environmental changes such as air pollution caused by intensified economic activity has increased the demand for goods and services that reduce environmental impact (Garnett, 2008; Hariga et al., 2017). To remain competitive, current supply chains must be re-designed and become more eco-efficient. In other words, they need to decrease the environmental impact for the same or even lower cost (Banasiket al., 2016). A major source of inefficiency of current supply chains is related directly to transportation, which is responsible for $14 \%$ of total $\mathrm{CO}_{2}$ emissions both at global and EU level (Dekker et al., 2012). Cooperative logistics has been suggested as a way of reducing $\mathrm{CO}_{2}$ emissions and of improving sustainability in supply chains (McKinnon, 2016; Ramanathan et al., 2014; Vanovermeire et al., 2014; Chen et al., 2017). This is mainly because cooperation improves the utilisation rate of vehicles at it allows cooperating companies to exploit the synergies between them (Cruijssen et al., 2007a). A report from 2012 showed that the average loading rate of vehicles is $56 \%$ in terms of weight, and combining loads could thus decrease the number of vehicles driving around, which can help reducing costs and $\mathrm{CO}_{2}$ emissions (Cruijssen, 2012).

Vendor-managed inventory (VMI) is a form of cooperation in which a vendor manages its own inventory as well as the inventory of its customer(s) by taking decisions on the replenishment quantity and frequency. The vendor is also responsible for keeping the stock level of the customer within agreed limits (Nagarajan and Sošić, 2008). VMI cooperation can result in substantial economic and environmental benefits because both transportation and inventory planning decisions can be optimised jointly (Stellingwerfet al., 2018b). Successful long term VMI that aims to improve eco-efficiency of the supply chain requires a fair distribution of economic benefits or costs based not only on the contribution of each participant to the shared cost savings but also to the shared environmental savings (Stellingwerfet al., 2018b).

Cooperative game theory (CCT) methods have been used to identify a fair allocation of the benefits of cooperation in supply chains. CCT methods generally focus on allocation of economic benefits based on the contribution of the participants to improve the economic performance of the cooperation (Frisket al., 2010). Recently, a commonly used CCT method, the Shapley value (Shapley, 1953) has been applied to allocate emissions in cooperative transportation (Naber et al., 2015). However, the possibility to use CGT methods to allocate the economic benefits based on both the economic and environmental contribution of participants has not been explored; CCT methods have mainly been used either for economic or for environmental allocation (Cuajardo, 2018). The objective of this Chapter is to propose a methodology for allocating economic benefits of VMI based on both the economic and environmental contributions of participants, such that both contributions are rewarded and eco-efficient forms of cooperation are stimulated. The proposed methodology is used to distribute economic benefits of VMI cooperation in supermarket chains in the Netherlands. The remainder of the paper is organised as follows. Section 5.2 provides the theoretical framework; Section 5.3, describes the proposed methods; Section 5.4 describes a case study; Section 5.5 describes the results; and in Section 5.6, the 
main conclusions are presented.

\subsection{Theoretical framework}

\section{Quantifying benefits of cooperation}

Literature has shown that logistics cooperation can bring significant benefits to cooperating partners (Cruijssen et al., 2007a), such as cost reductions and efficiency gains (AdensoDíaz et al., 2014). The review of Chen et al. (2017) suggests that until recently, cooperation studies have focused on monetary benefits but there is a trend towards measuring or estimating the environmental impacts of supply chain cooperation as well. For example, Ramanathan et al. (2014) qualitatively evaluated how the environmental pressure from different stakeholders can help to improve supply chain cooperation, which in turn can lead to improved business performance.

Cooperative benefits have also been studied qualitatively. Stellingwerfet al. (2018b) have evaluated different forms of cooperation and they have quantitatively shown that VMI is an advanced form of cooperation that can provide significant cost and emission savings. In order to quantify benefits of VMI, models based on the inventory routing problem (IRP) can be used because in these models routing and inventory decisions are optimised simultaneously (Coelho et al., 2013). In general, IRP models minimise cost, which are calculated by adding inventory, transportation, and labour cost. Stellingwerfet al. (2018b) used an IRP model to quantify both the economic and environmental effects of cooperation in fresh food logistics. This model will be used in this Chapter as well; it will be briefly explained in the next section and completely formulated in the Appendix. It was found that VMI can bring significant benefits to partners, since both transportation and inventory are optimised. After total cooperative benefits have been quantified, they have to be distributed among the partners, and this is the role of cooperative game theory.

\section{Allocation of cost and $\mathrm{CO}_{2}$ emissions in supply chain cooperation}

In order to ensure that a cooperation is successful in the long run, it is important that the associated cost and the resulting gains are allocated in a way that is considered fair by all participants (Cruijssen et al., 2007c). CGT methods have been used to identify potential coalitions, to quantify the total benefits of these coalitions, and to fairly allocate the benefits of the coalitions to all cooperating partners (Nagarajan and Sošić, 2008). In most CCT methods, the benefits or cost allocated to a partner are related to the partner's contribution to the group's cost savings (Guajardo and Rönnqvist, 2016). Different CGT methods exist and Tijs and Driessen (1986) have summarised them. The most common methods are the Shapley value, the nucleolus, the equal charge method, the alternative cost avoided method, and the cost gap method. Recent case studies that compare different cost allocation methods are, for example, Frisk et al. (2010), Vanovermeire et al. (2014), and Wang et al. (2017). Frisk et al. (2010) applies different cost allocation methods (the equal profit method that they developed themselves and the and Shapley value based methods) to a case study on cooperative wood transportation by different forest companies. They find 
similar allocations in both methods, but they argue that the equal profit method is easier to understand. Vanovermeire and Sörensen (2014) argue that flexibility is crucial in horizontal logistics cooperation, and they test different allocation methods on their ability to reward flexibility of cooperating partners. They discuss that the Shapley value, the nucleolus and the equal charge method give consistent and fair incentives for flexibility. Wang et al. (2017) allocate cooperative vehicle routing cost savings using the Shapley value.

The above-mentioned studies focus on transport cooperation. Allocation of benefits in VMI cooperation (joint optimisation of both transportation and inventory decisions) has only been studied in the work of Özener et al. (2013). They compare different cost allocation methods for VMI cooperation in a case study where cost-to-serve has to be split between clients. They propose different methods which perform better than proportional allocation methods, which have often been discussed to not result in fair allocations. However, this Chapter does not consider environmental effects of VMI cooperation, and neither does it study how those environmental benefits could be used in allocation decisions.

There are some gain allocation studies that conclude that logistics cooperation results in cost savings as well as environmental benefits (Frisk et al., 2010; Vanovermeire et al., 2014; Jonkman et al., 2019). Other recent studies have started to use gain allocation methods to allocation $\mathrm{CO}_{2}$ emissions to cooperating partners (Kellner and Otto, 2012; Naber et al., 2015; Zhu et al., 2016). Some authors include $\mathrm{CO}_{2}$ emissions as part of the cost function, on which they apply different allocation methods (Özener, 2014; Niknamfar and Niaki, 2016; Sanchez et al., 2016). Guajardo (2018) studied cooperative logistics in a cost minimising as well as in an emission minimising setting. In a small 3-partner problem, costs and emissions are minimising separately. For the cost minimising solution, costs are allocated using the proportional method, the Shapley method, and the Nucleolus. And for the emission minimising solutions, emissions are allocated using the same methods. Despite the small problem size, the optimal solution for cost and emission minimisation is different, a different optimal route is found.

Despite the efforts that have been done to quantify and distribute the benefits of cooperation, the contribution of a partner to reduce the $\mathrm{CO}_{2}$ emissions of the cooperation is has not been translated to economic benefits, which is the gap this Chapter attempts to address. In order to engage partners in the improvement of the eco-efficiency of their supply chain through cooperation, the partners should not only be rewarded based on their contribution to cost savings of the coalition but also based on their contribution to emissions savings. Table 5.1 summarises the studies discussed in the theoretical framework.

From Table 3.1, it can be seen that the Shapley value is a the most commonly used CCT method in the discussed studies. According to Guajardo and Rönnqvist (2016), who review gain allocation methods in cooperative transportation, this is because the Shapley value satisfies important fairness properties. Moreover, despite the fact that the Shapley solution is not guaranteed to be in the core (i.e. the gain allocation of all players within a cer- 
Table 5.1: Overview of literature discussed. Abbreviations: JRP, joint route planning; ECM, equal charge method; ACAM, alternative cost avoided method; EPM, equal profit method; CVMI, cooperative vendor-managed inventory.

\begin{tabular}{|c|c|c|c|c|}
\hline Authors & $\begin{array}{l}\text { Form of } \\
\text { cooperation }\end{array}$ & $\begin{array}{l}\text { Economic } \\
\text { quantification }\end{array}$ & $\begin{array}{l}\text { Environmental } \\
\text { quantification }\end{array}$ & $\begin{array}{l}\text { Allocation methods } \\
\text { studied }\end{array}$ \\
\hline Kellner and Otto (2012) & JRP & no & yes & $\begin{array}{l}15 \text { different ones including } \\
\text { Shapley value }\end{array}$ \\
\hline Nagarajan and Sošić (2008) & CVMI & yes & no & Nash bargaining problem \\
\hline Frisket al. (2010) & JRP & yes & no & $\begin{array}{l}\text { Volume, Shapley, shadow, ECM } \\
\text { ACAM, nucleolus, EPM }\end{array}$ \\
\hline Özeneret al. (2013) & CVMI & yes & no & $\begin{array}{l}\text { Duality based methods, } \\
\text { Shapley value }\end{array}$ \\
\hline Özener (2014) & JRP & yes & yes & $\begin{array}{l}\text { duality and Shapley value based } \\
\text { allocation mechanism }\end{array}$ \\
\hline Naberetal. (2015) & JRP & no & yes & $\begin{array}{l}\text { Proportional, star, Shapley } \\
\text { Nucleolus, Lorenz, EPM }\end{array}$ \\
\hline Niknamfar and Niaki (2016) & JRP & yes & yes & $\begin{array}{l}\text { dual lexicographic max-min } \\
\text { approach }\end{array}$ \\
\hline Sanchez et al. (2016) & JRP & yes & yes & Shapley value \\
\hline Zhuet al. (2016) & JRP & no & yes & $\begin{array}{l}\text { Shapley, transport work based, } \\
\text { distance based, volume based }\end{array}$ \\
\hline Palhazi Cuervo et al. (2016) & JRP & yes & no & Proportional \\
\hline
\end{tabular}

tain coalition is better compared to their allocation in all other possible smaller coalitions or the non-cooperative solution) in most of the studies discussed in the review of Cuajardo and Rönnqvist (2016), the Shapley was found to be a core solution. Finally, Cruijssen et al. (2010) propose Shapley as a practical CCT method because it is easy to interpret and communicate with the decision makers. In the study of Vanovermeire and Sörensen (2014), the Shapley value was used in a case study and it was found that it gave incentives to cooperate. Because of the appealing properties of the Shapley value and its broad use in CCT literature, it is attractive from a practical as well as a scientific point of view. Therefore, this Chapter uses the Shapley value as well.

\section{Benefit allocation using the Shapley value}

The Shapley value (Shapley, 1953) is one of the most common gain sharing rules in the literature since it provides a unique solution to the allocation problem and it satisfies appealing fairness properties (Lozano et al., 2013). Almost all studies discussed use (amongst others) the Shapley value. The Shapley method will be used in this Chapter as it will allow for comparison with the other studies. The Shapley value provides an allocation for all cooperative partners based on each partner's contribution to the total costs or benefits of the group and it is calculated as:

$$
y_{j}=\sum_{S \subseteq N \backslash\{j\}} \frac{(|S|-1) !(|N|-|S|) !}{|N| !}[c(S)-c(S-\{j\})],
$$

where $N$ is the group in which all partners participate, which is called grand coalition. $S$ is any sub-coalition by the partners of the grand coalition, and $y_{j}$ is value allocated to partner $j . C(S)$ is the value (e.g. costs or $\mathrm{CO}_{2}$ emissions) of coalition $S, c(S-\{j\})$ is the value of coalition $\mathrm{S}$ without partner $j$. Therefore, $c(S)-c(S-\{j\})$ is the amount by which the value of coalition $c(S-\{j\})$ increases when participant $j$ joins the coalition, 
i.e. the marginal value of participant $j$. The Shapley value allocated to a partner (Eq. 5.1) can be interpreted as the average marginal contribution that partner brings to the group (Shapley, 1953). In order to calculate the Shapley value, it is necessary to know the value of all possible sub coalitions that can be formed from the grand coalition. These values are called the characteristic function. For more information on the Shapley value, the interested reader is referred to Shapley (1953).

\subsection{Methodology}

In order to establish an allocation based on both cost and $\mathrm{CO}_{2}$ emissions, a three-step methodology is proposed, summarised in Figure 5.1. In the first step, the set of eco-efficient solutions is calculated using the green IRP model proposed by Stellingwerfet al. (2018b) because it optimises inventory and routing decisions simultaneously by minimising either the environmental impact or the costs of a potential coalition. The IRP is an extension of the better-known Vehicle Routing Problem (VRP). In the basic VRP model, the objective is to identify the shortest route for visiting a set of individual customers. Cenerally, travelling distance is minimised given (a) vehicle(s) that has to leave and return to a depot and deliver demand to a number of customers. Constraints are used to limit the carrying capacity of the vehicles. Lately, variants of the VRP have been developed where the objective function is formulated such that costs and/or emissions can be minimised instead of distance. A VRP can be used to solve one-period routing problems. In the IRP, not only routing, but also inventory decisions are optimised simultaneously. The constraints of the IRP are the same as the VRP, but constraints that define the inventory at the depot and the demand locations are added, as well as inventory capacity constraints. The IRP can be used for multi-period routing and inventory problems: if there is enough inventory in the demand locations, less frequent routing with higher volumes could be considered.

In order to estimate emissions $E\left(\mathrm{~kg} \mathrm{CO}_{2}\right)$ using an IRP model, the fuel use is calculated since it is assumed to be linearly related to $\mathrm{CO}_{2}$ emissions. Fuel use in road transport depends on the weight carried, the slope of the road, the distance travelled, the air density, and the vehicle speed (Bektaş and Laporte, 2011). The cost $C(€)$ is calculated by adding wage cost, fuel cost, and inventory holding cost.

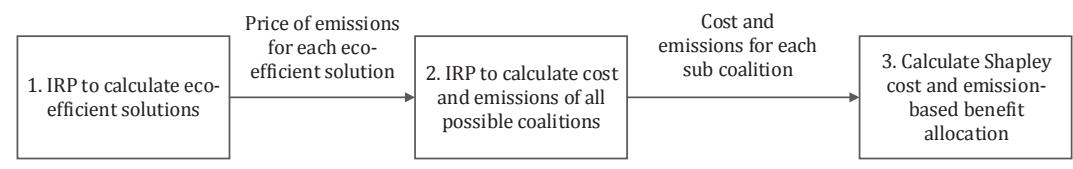

Figure 5.1: Summary of the methodology used in this Chapter to arrive to a cost and $\mathrm{CO}_{2}$ emissions based Shapley allocation.

The IRP constraints can be subdivided in three main types: (i) inventory constraints: they impose minimum and maximum levels of the inventory of the depot and the destina- 
tions in each period considering an initial inventory level; (ii) vehicle routing constraints: they impose that a delivery can be made to a location only if it is visited by a vehicle, and that a vehicle cannot visit the same location multiple times in the same time period, they restrict the number of vehicles used per day, and they define vehicle capacity; (iii) flow constraints: these define how the load of the vehicle changes after visiting a location, eliminate sub tours, and define impossible arcs. The mathematical formulation of the IRP model and the calculations of the cost and $\mathrm{CO}_{2}$ emissions are presented in the Appendix. In that section, the parameters used in the IRP model are also summarised, and the values used to run the model are given. The IRP model was coded and solved with Fico Xpress Mosel version 8.0 on a PC with Intel Core i5 processor $(2.6 \mathrm{GHz})$ and eight $\mathrm{CB}$ of RAM memory.

In the first step, in order to find eco-efficient solutions, the weighing method proposed by Romero and Rehman (2003) is used, which is a commonly used method in goal programming. This is a convenient method as the $\lambda$ value needed for the weighing method can also be used later in the gain allocation method. An additive objective function is formulated (Eq. 5.2) subject to the constraints of the above mentioned IRP model.

$$
\text { Minimise } K=C+\lambda E \text {, }
$$

where $K$ is the additive objective function value (€), $C$ is the cost ( $€$ ), $\lambda$ is the price of $\mathrm{CO}_{2}$ emissions ( $€ / \mathrm{kg}$ ), which can also be interpreted as the relative importance of saving $\mathrm{CO}_{2}$ emissions as compared to saving cost, $E$ are the $\mathrm{CO}_{2}$ emissions (kg). By varying $\lambda$, the alternative optimal (eco-efficient) solutions can be found.

The set of eco-efficient solutions is calculated by changing the values of $\lambda$. The higher the value of $\lambda$, the more importance is attached to the environmental indicator $E$. The model is run in an iterative way, starting with $\lambda$ (which results in the cost minimising solution) and the value of $\lambda$ is increased in steps of 0.1 until the emission minimising solution is found. The value of lambda leading to an eco-efficient solution is used as the price of emissions when calculating the benefit allocation of that eco-efficient solution. If there is a range of $\lambda$ values leading to the same solution, the average of that range $\bar{\lambda}$ is used for the gain allocation step.

In the second step, the $\bar{\lambda}$ value of each eco-efficient solution is used in the IRP model to calculate the minimum K-value for each possible sub-coalition that can be formed from the cooperating participants. This way, for each $\bar{\lambda}$ value, the characteristic function of the Shapley value (Eq. 5.1) is calculated.

In step three, the characteristic function is used to allocate the K-value benefits to the partners using the Shapley value (see Eq. 5.1). The K-value can be converted back to costs, which are easier to interpret, using the following equation:

$$
y_{j}^{c}=\frac{y_{j}^{k} \times C}{K} \text {, }
$$


where $y_{j}^{k}$ is the K-value allocated to partner $j(€), y_{j}^{c}$ is the cost allocated to partner $j$ $(€)$, With this procedure, the total costs of an eco-efficient solution are divided over the partners. No extra costs are paid for emissions, but when $\lambda$ chosen such that the costminimising solution is not optimal, the contributions of the partners to saving emissions are also considered in the cost allocation. The higher the value of $\lambda$, the higher the advantage for partners that help save emissions.

After this three-step procedure, all eco-efficient solutions, the prices of $\mathrm{CO}_{2}$ emissions that lead to that solutions, and the allocations based on those emissions prices are calculated. The cooperative costs (savings) are allocated among the partners, but the cooperative emissions are not since the partners have agreed to regard that as a group benefit: if the group saves $40 \%$ of emissions, all partners can claim that they save $40 \%$ of emissions by cooperating. In this Chapter, the allocation of benefits based on both costs and emissions is done using the Shapley value, but other gain allocation methods could be used as well.

The generation of all possible sub-coalitions of step 2 as well as the Shapley value calculations of step 3 were coded in R (version i386 3.2.1) on a PC with Intel Core i5 processor $(2.6 \mathrm{CHz})$ and eight $\mathrm{CB}$ of RAM memory.

\subsection{Case study: VMI for eco-efficient food distribution in the Netherlands}

\section{Data and assumptions}

The case study is based on seven supermarket chains in the Netherlands. Currently, they cooperate by buying their products together in order to negotiate a lower unit price. The cooperatively bought products are delivered to their shared central distribution centre (CDC, denoted by 0 ). From there, the supermarket chains arrange their logistics separately. They individually pick up (or use a logistics service provider to pick up) the products from the CDC and bring them to their distribution centres (DCs, denoted by 1-7). The supermarket chains aim to further cooperate by implementing VMI between the CDC and the DCs to reduce cost and $\mathrm{CO}_{2}$ emissions. They consider to allocate the resulting economic gains by accounting for their contribution to reducing both cost and $\mathrm{CO}_{2}$ emissions of the coalition.

The distances between the CDC and the DCs are presented in Table 5.2. The aggregate weekly demand of a representative set of fresh food products of each DC (in $\mathrm{kg}$ ) is presented in Table 5.3. Note that there is no demand on Sunday because the CDC and the DCs are closed on that day. On some days, there is no demand (the zeroes in Table 5.3) because some DCs do not place orders every day. Other values used to run the IRP model can be found in Table A.3 in the Appendix. 
Table 5.2: Distances (in km) between the DCs of the supermarket chains (denoted by 1-7) and the $\mathrm{CDC}$ (denoted by 0 ).

\begin{tabular}{r|rrrrrrrr} 
DC & 0 & 1 & 2 & 3 & 4 & 5 & 6 & 7 \\
\hline 0 & 0 & 91 & 6 & 134 & 82 & 117 & 74 & 192 \\
1 & 91 & 0 & 91 & 75 & 134 & 43 & 20 & 155 \\
2 & 6 & 91 & 0 & 134 & 84 & 117 & 75 & 194 \\
3 & 134 & 75 & 134 & 0 & 168 & 46 & 94 & 90 \\
4 & 82 & 134 & 84 & 168 & 0 & 158 & 140 & 180 \\
5 & 117 & 43 & 117 & 46 & 158 & 0 & 59 & 117 \\
6 & 74 & 20 & 75 & 94 & 140 & 59 & 0 & 174 \\
7 & 192 & 155 & 194 & 90 & 180 & 117 & 174 & 0
\end{tabular}

Table 5.3: Aggregate demand in kg at each DC (1-7).

\begin{tabular}{r|rrrrrr} 
& Monday & Tuesday & Wednesday & Thursday & Friday & Saturday \\
\hline 1 & 540 & 540 & 450 & 450 & 810 & 0 \\
2 & 1890 & 1680 & 1260 & 2760 & 2220 & 1170 \\
3 & 270 & 630 & 360 & 540 & 1440 & 0 \\
4 & 0 & 1122 & 0 & 1248 & 0 & 0 \\
5 & 1260 & 810 & 1350 & 1260 & 540 & 1080 \\
6 & 1170 & 1524 & 1170 & 1608 & 2412 & 0 \\
7 & 0 & 720 & 360 & 720 & 360 & 450
\end{tabular}

\section{Setup of calculations}

In order to establish a Shapley allocation based on both the environmental and the economic contributions of the partners, the three-step method described in Figure 5.1 is followed. First, the data described above are input to an IRP model (Stellingwerfet al., 2018b) with the aggregate objective function (Eq. 5.2) to find the eco-efficient cooperative solutions (Romero and Rehman, 2003). To find all these solutions, the IRP model is run iteratively with an increasing value of $\lambda$, with a step size of 0.1. The weighing method (which uses an additive objective functions) results in an optimal solution for each $\lambda$. As these $\lambda$ values directly correspond with the price of emissions, it can also be used in the rest of the calculations. For each eco-efficient solution, the corresponding average price of emissions $(\bar{\lambda})$ is determined. Second, the IRP model is solved for all possible sub-coalitions and for all different eco-efficient solutions (using the different values of $\bar{\lambda}$ ). And third, the Shapley value is calculated based using the characteristic function calculated in step 2. The Shapley allocation based on the K-value is translated to the actual cost. Finally, the savings in terms of the additive objective function value $\mathrm{K}$, and in terms of cost for all groups and for all individuals are calculated using Eq. (5.3).

To assess the potential benefits of VMI cooperation the allocations of each eco-efficient solution are compared to the current situation. To estimate the current allocations, costs are minimised using the IRP model while imposing that each supermarket chain optimises transportation separately. To better understand the impact of important model parameters such as the distance between DCs and CDC and the demand of the SC one of the eco-efficient solutions found is used to test a set of alternative scenarios is explored. These scenarios test the effect of distance and demand. Table 5.4 summarises the scenarios. In this table, base refers to one of the solutions found optimising the additive objective function given the demand and distance data in Table 5.2 and 5.3. In this table 
equal distance refers to the situation where all partners are located equally far from the CDC, but the total distance of the matrix is still the same as in the base case scenarios (illustrated in Figure 5.2). Equal demand refers to the situation where the total demand of all partners is equal ( $1 / 7$ of the group demand), but the week structure is kept (i.e. if $14 \%$ of the total demand is on Monday in the base case, that is still the case in the equal demand situations).

Table 5.4: The scenarios used to assess the impact of distance and demand.

\begin{tabular}{cll}
\hline Scenario & Distance & Demand \\
\hline 1 & Base & Base \\
2 & Equal & Equal \\
3 & Base & Equal \\
4 & Equal & Base \\
\hline
\end{tabular}

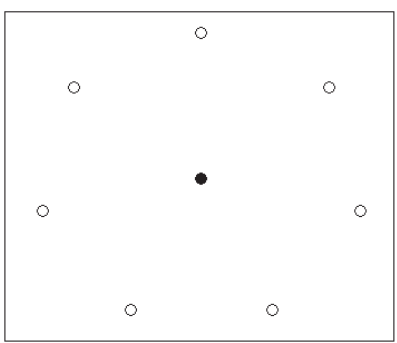

Figure 5.2: Uniform distance distribution of locations. Black point indicates $C D C$, white points indicate DCs.

\subsection{Results and discussion}

\section{Analysis of the eco-efficient solutions}

Using the IRP model with the additive objective function, the eco-efficient solutions were calculated. Figure 5.3 shows the cost and $\mathrm{CO}_{2}$ emissions of all calculated eco-efficient solutions and of the current situation: 11 discrete eco-efficient cooperative solutions were calculated. All this solutions are optimal and depend on the importance allocated to the two objectives through the value of lambda. To find these solutions, the model was run in multiple iterations with a range of lambda values (0-40 with a step size of 0.1 ). Values of $\lambda$ above 31.6 result in the emission-minimising solution. For all other solutions, there was a range of $\lambda$ values leading to that solution.

The 11 eco-efficient solutions found were labelled as $s 1$ for the emission minimising solution to $s 11$ for the cost-minimising solution. Table 5.5 shows the $\mathrm{CO}_{2}$ emissions, costs, distance, travelling time and the number of trips related to each eco-efficient solution, as well as the current situation. Since there was a range of lambdas leading to the different solutions, only the averages of each solution range are shown. 


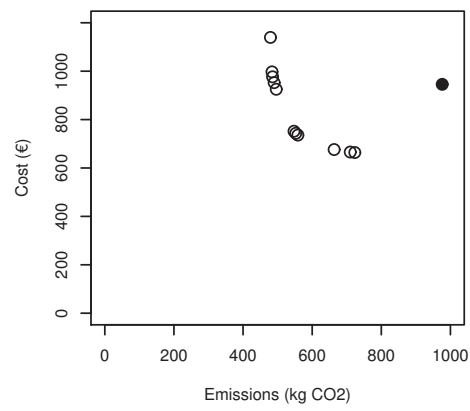

Figure 5.3: $\mathrm{CO}_{2}$ emissions $(\mathrm{kg})$ and cost $(€)$ for the cooperative solution options and the current situation.

Table 5.5: Current solution and the eco-efficient solutions. Abbreviations: $E$, emissions; $C$, costs; $C_{t}$, transportation costs; $C_{I}$, inventory costs; $D$, distance; $T$, travelling time; $n$, number of trips.

\begin{tabular}{|c|c|c|c|c|c|c|c|c|c|c|c|c|}
\hline & current & s1 & s2 & s3 & s4 & s5 & s6 & s7 & s8 & s9 & $\mathrm{s} 10$ & s11 \\
\hline $\bar{\lambda}$ & 0 & 31.6 & 22.25 & 9.15 & 4.95 & 3.95 & 2.60 & 1.50 & 0.90 & 0.45 & 0.25 & 0.10 \\
\hline$E(\mathrm{~kg})$ & 976 & 479 & 484 & 485 & 490 & 496 & 547 & 552 & 559 & 664 & 710 & 723 \\
\hline$C(€)$ & 946 & 1139 & 997 & 977 & 953 & 925 & 752 & 743 & 736 & 676 & 666 & 664 \\
\hline$-C_{t}(€)$ & 512 & 252 & 254 & 255 & 257 & 260 & 287 & 290 & 293 & 348 & 373 & 380 \\
\hline$-C_{I}(€)$ & 433 & 888 & 743 & 722 & 695 & 665 & 465 & 453 & 443 & 328 & 293 & 284 \\
\hline$D(\mathrm{~km})$ & 1837 & 808 & 816 & 820 & 828 & 841 & 931 & 938 & 950 & 1168 & 1215 & 1241 \\
\hline$T(\mathrm{~h})$ & 29.0 & 13.0 & 13.3 & 13.4 & 13.8 & 14.2 & 16.2 & 16.5 & 16.9 & 20.5 & 21.6 & 22.2 \\
\hline$n$ & 14 & 4 & 4 & 5 & 5 & 6 & 6 & 6 & 7 & 8 & 7 & 8 \\
\hline
\end{tabular}

Table 5.5 shows that all cooperative eco-efficient solutions result in less $\mathrm{CO}_{2}$ emissions compared to the current situation. Solutions $s 5$ to $s 11$ correspond to lower costs than the current situation. Moreover, minimising $\mathrm{CO}_{2}$ emissions results in a solution in which products are delivered less frequently. On the other hand, minimising cost results in a solution in which the companies are supplied more frequently, since this reduces their inventory cost. This is also reflected in the decrease in transportation cost and an increase in inventory cost when the price of $\mathrm{CO}_{2}$ emissions $(\bar{\lambda})$ increases. Moreover, it is illustrated by the change in the number of trips, the distance driven and the driving time.

\section{Shapley allocation of the K-value and costs}

Table 5.6 provides the current uncooperative costs and $\mathrm{CO}_{2}$ emissions, as well as the total savings in $\mathrm{CO}_{2}$ emissions for each solution and the cost allocation based on the K-value for each participant in each eco-efficient solution. Also, it shows the percentage allocated to each partner in each solution. Since Eq. (5.3) is used to convert the K-allocation to costs using a fixed factor (the K-allocation of a partner divided by the total K-value), the percentage of total allocation will be the same whether this is cost, emissions or K-value. 
Table 5.6: Current costs ( $€$ ) and emissions ( $\mathrm{kg} \mathrm{CO}$, and total emission savings (\%) costs ( $€$ ) and percentage of the K-value (\%) allocated for all eco-efficient solutions.

\begin{tabular}{|c|c|c|c|c|c|c|c|c|c|c|c|c|c|c|}
\hline Solution & \multicolumn{2}{|c|}{ Individual } & \multirow{2}{*}{\multicolumn{2}{|c|}{$\begin{array}{c}\text { s1 } \\
51 \%\end{array}$}} & \multicolumn{2}{|c|}{ s2 } & \multicolumn{2}{|c|}{ s3 } & \multicolumn{2}{|c|}{ s4 } & \multicolumn{2}{|c|}{ s5 } & \multicolumn{2}{|c|}{ s6 } \\
\hline E saving & & & & & & & & & & & & & & \\
\hline Partner & C & $\mathrm{E}$ & c & K & c & K & c & $\mathrm{K}$ & c & $\mathrm{K}$ & c & K & c & $\mathrm{K}$ \\
\hline 1 & 113 & 96 & 106 & 9 & 92 & 9 & 89 & 9 & 87 & 9 & 84 & 9 & 66 & 9 \\
\hline 2 & 58 & 39 & 38 & 3 & 36 & 4 & 45 & 5 & 54 & 6 & 55 & 6 & 48 & 6 \\
\hline 3 & 166 & 143 & 170 & 15 & 148 & 15 & 143 & 15 & 138 & 14 & 133 & 14 & 109 & 14 \\
\hline 4 & 75 & 86 & 177 & 16 & 153 & 15 & 143 & 15 & 131 & 14 & 124 & 13 & 97 & 13 \\
\hline 5 & 208 & 249 & 189 & 17 & 167 & 17 & 167 & 17 & 167 & 18 & 165 & 18 & 137 & 18 \\
\hline 6 & 168 & 162 & 165 & 15 & 147 & 15 & 152 & 16 & 155 & 16 & 154 & 17 & 129 & 17 \\
\hline 7 & 158 & 202 & 294 & 26 & 254 & 25 & 237 & 24 & 220 & 23 & 210 & 23 & 165 & 22 \\
\hline sum & 946 & 976 & 1139 & 100 & 997 & 100 & 977 & 100 & 953 & 100 & 925 & 100 & 752 & 100 \\
\hline
\end{tabular}

Table 5.6: Current cost $(€)$, and costs $(€)$ and percentage of the K-value (\%) allocated for all ecoefficient solutions (continued).

\begin{tabular}{|c|c|c|c|c|c|c|c|c|c|c|c|c|}
\hline Solution & \multicolumn{2}{|c|}{ Individual } & \multicolumn{2}{|c|}{ s7 } & \multicolumn{2}{|c|}{ s8 } & \multicolumn{2}{|c|}{ s9 } & \multicolumn{2}{|c|}{$s 10$} & \multicolumn{2}{|c|}{ s11 } \\
\hline \multirow{2}{*}{ E saving } & \multirow[b]{2}{*}{ C } & \multirow[b]{2}{*}{$E$} & \multicolumn{2}{|c|}{$43 \%$} & \multicolumn{2}{|c|}{$43 \%$} & \multicolumn{2}{|c|}{$32 \%$} & \multicolumn{2}{|c|}{$12 \%$} & \multicolumn{2}{|c|}{$26 \%$} \\
\hline & & & C & $\mathrm{K}$ & C & $\mathrm{K}$ & C & $\mathrm{K}$ & C & $\mathrm{K}$ & C & $\mathrm{K}$ \\
\hline 1 & 113 & 96 & 64 & 9 & 66 & 9 & 61 & 9 & 71 & 9 & 58 & 9 \\
\hline 2 & 58 & 39 & 52 & 7 & 54 & 7 & 51 & 8 & 64 & 8 & 55 & 8 \\
\hline 3 & 166 & 143 & 109 & 15 & 111 & 15 & 103 & 15 & 124 & 15 & 103 & 16 \\
\hline 4 & 75 & 86 & 92 & 12 & 86 & 12 & 77 & 11 & 89 & 11 & 72 & 11 \\
\hline 5 & 208 & 249 & 139 & 19 & 141 & 19 & 132 & 20 & 161 & 20 & 132 & 20 \\
\hline 6 & 168 & 162 & 130 & 18 & 128 & 17 & 115 & 17 & 138 & 17 & 113 & 17 \\
\hline 7 & 158 & 202 & 158 & 21 & 150 & 20 & 137 & 20 & 162 & 20 & 132 & 20 \\
\hline sum & 946 & 976 & 744 & 100 & 736 & 100 & 676 & 100 & 808 & 100 & 664 & 100 \\
\hline
\end{tabular}

The percentage of benefits allocated to partner 1 remains the same in all calculated ecoefficient solutions; partner 1 may equally contribute to saving cost and to saving $\mathrm{CO}_{2}$ emissions. For partner 2, 5, and 6 there is an increase in the allocation when moving from s1, the $\mathrm{CO}_{2}$ minimising solution, to $\mathrm{s} 11$, the cost minimising solution. Apparently partner 2 , 5 , and 6 are more helpful in saving $\mathrm{CO}_{2}$ compared to the other partners. These partners have a relatively high demand, but not too high to limit to possibility to combine their demand with other demands. Moreover, they are located close to the CDC and their demand is spread well over the week. These aspects make it easy to combine these partners in cooperative routes.

For partner 3 , the emission allocation is relatively stable from $s 1$ to $s 5$ but from $s 6$ to $s 11$, when the price of $\mathrm{CO}_{2}$ increases further, partner 3 is allocated an increased amount of emissions. Apparently partner 3 does not contribute that much to saving emissions in comparison to the other partners. This might relate to the fact that partner 3 is located relatively far from the CDC. The cost allocation results (Table 5.6) show that partner 4 does not save cost in any solution except for solution $s 11$, the cost minimising solution. The demand of partner 4 is relatively low, and this partner only has demand on two days of the week. This might limit the opportunities for making cooperative route, which is why the benefits allocated to this partner are also relatively low. For solution s1 to s8 (when the price of emissions is higher than $0.90 € / \mathrm{kg}$ ), partner 7 is faced with a cost increase. Partner 7 is located the furthest from the CDC, which is why this partner does not contribute much to saving emissions, and why this partner only benefits when the price 
of emissions is low.

\section{The effects of distance and demand on the allocation}

To assess the impact of different demand and distance scenarios on the calculated allocation, the eco-efficient solution $s 8$, which has a $\bar{\lambda}$ value of 0.9 , was further analysed. The total cost and $\mathrm{CO}_{2}$ emissions of this solution are $€ 559$ and $736 \mathrm{~kg}$, respectively. This solution is a non-extreme, balanced solution between cost and $\mathrm{CO}_{2}$ emissions. These scenarios could also be tested on any other eco-efficient solution. Table 5.7 provides the resulting allocations.

Table 5.7: The benefit allocations (\%) resulting from the distance and demand scenarios.

\begin{tabular}{cllrrrrrrr}
\hline & & \multicolumn{7}{c}{ Partner } \\
\hline Scenario & Distance & Demand & 1 & 2 & 3 & 4 & 5 & 6 & 7 \\
\hline 1 & Base & Base & 9.0 & 7.3 & 15.1 & 11.6 & 19.2 & 17.4 & 20.4 \\
2 & Equal & Equal & 14.3 & 14.3 & 14.3 & 14.3 & 14.3 & 14.3 & 14.3 \\
3 & Base & Equal & 12.0 & 4.1 & 16.3 & 17.6 & 14.0 & 11.3 & 24.7 \\
4 & Equal & Base & 10.2 & 22.9 & 12.0 & 8.5 & 16.6 & 19.5 & 10.3 \\
\hline
\end{tabular}

When all distances to the DC are equal and all demands are equal, the resulting allocation is equal for every partner (Table 5.7). This result is inherent to the Shapley value, because it has the symmetry property: if a partner contributes the same as an other partner, the allocation of those partners should be the same. Partner 4 and 7 do not always benefit from the allocation (see Table 5.6). The results of the demand and distance scenarios can help explain why this is the case. In the base case, partner 4 is relatively far from the other DCs. Therefore, the equal-distance cases cause a lower allocation for partner 4. Partner 4 also has a low demand and only has demand on two days in the base case. This probably causes partner 4 to have a limited number of cooperation possibilities which is why this partner can also not benefit that much from cooperation. Note that the relative allocation to partner 4 is not high but since the demand is low, the allocation per unit is relatively high.

Partner 7 is located very far from the CDC in the base case. Therefore, a reduction in distance (as in the equal distance scenarios) also reduces the allocated percentage of this partner.

\subsection{Conclusions and future work}

This study developed a method to allocate benefits of costs and emissions according to contributions of the cooperating partners. A case study on VMI cooperation between Dutch supermarket chains demonstrated how the method works. The results of the case study showed a trade-off between costs and emissions, and multiple alternative cooperative solutions. Different emissions prices were used to determine the different solutions. In turn, emissions prices were used to allocate the monetary benefits. Most of the solutions resulted in savings for all partners. However, some partners were confronted with cost increases. Those partners have demand or location characteristics that make cooperation with the other partners non-beneficial. In practise, those partners might choose 
to continue individually organising their logistics, or to cooperate with partners outside the group used in the case study. The results have shown that the economic benefits of cooperation can be fairly divided, while simultaneously rewarding the effort of partners that reduce $\mathrm{CO}_{2}$ emissions and costs.

Prior to the case study, the partners were already cooperating to a limited extent. The partners arranged their procurement together and were co-owners of a shared distribution centre. Therefore, CCT methods were a logical next step in distributing benefits. In other similar cases where pre-existing forms of cooperation al ready exist, CCT methods are an appropriate approach for allocating cooperative benefits. Guajardo et al. (2018) studied coalition formation among cooperative agents. When there are many possible cooperative partners, it is useful to combine their techniques with CCT. Future research or other case studies could focus on situations with no pre-existing form of cooperation. In those situations, non-cooperative game theory could be used to find coalitions with a low chance of being abandoned.

In the study, emission prices between 0 and $3.95 € / \mathrm{kg}$ led to a reduction of $\mathrm{CO}_{2}$ emissions without increasing costs. All cooperative solutions resulted in a decrease of $\mathrm{CO}_{2}$ emissions compared to the current situation, even when emissions were priced at $0 € / \mathrm{kg}$. Most of the emissions prices tested were much higher than the current price. For example, $3.95 € / \mathrm{kg}$ corresponds with $3950 € /$ ton whereas in the European Trading Scheme it has varied over the last 10 years between 4 and 40 euros per ton (CBS, 2018). However, the price of $\mathrm{CO}_{2}$ emissions used in this Chapter does not necessarily translate to an increase in costs; it mainly captures the importance a group attaches to savings attributed to $\mathrm{CO}_{2}$ emissions. It does influence the cost allocation based on the price of emissions.

The method proposed in the study can be applied to other kinds of cooperative partnerships. However, applying these methods to bigger cases can increase computation time for both the IRP model and for the Shapley value calculation. Heuristics for the IRP model and approximation methods for the Shapley value could reduce the calculation times. In this Chapter, the Shapley value is used to allocate benefits based on both costs and $\mathrm{CO}_{2}$ emissions, but the proposed three-step methodology can be used with other CCT methods as well. For example, the Equal Profit Method (Frisket al., 2010) and the methods described by Kellner and Otto (2012) could be useful for cases with more cooperating partners. Applying different CGT methods within the proposed framework can provide valuable information about the differing outcomes of cost and $\mathrm{CO}_{2}$ emissions based allocations. 
5.6. Conclusions and future work 


$$
6
$$




\section{Chapter 6}

\section{Conclusions and general discussion}

\subsection{Conclusions}

The overall objective of this PhD study was to develop decision support models that are able to capture the complexity of establishing eco-efficient food logistics cooperation in supply chains. To achieve this, we extended existing OR models to account for specific characteristics of food products such as perishability, and we quantified trade-offs between economic and environmental indicators. Different forms of cooperation were compared on their eco-efficiency performance, and a gain allocation method was adjusted such that it was not only based on costs but also on emissions. The main research question was:

Which decision support models can be used to design eco-efficient logistics cooperation in food supplychains?

To answer this question, four sub questions were formulated:

1. How to quantify eco-efficiency in temperature-controlled food logistics?

2. How to model temperature-dependent food quality decay in logistics models?

3. What are the effects of different forms of cooperation on eco-efficiency?

4. How can gain allocation be applied such that eco-efficient forms of logistics cooperation are stimulated?

Here, we summarise the main findings per research question. Then, scientific impact, societal and managerial implications, limitations of the study and future research directions are discussed.

\section{Eco-efficiency in temperature-controlled food supply chains}

In Chapter 2, it was found that estimation of costs and emissions of (fresh and frozen) food supply chains can be improved by considering the effects of temperature control. Temperature control in transportation can result in more emissions and costs, since extra fuel is used to drive the cooling engine of the vehicle. Next to that, in temperaturecontrolled transportation refrigerant slowly leaks out of the vehicle, which causes additional pollution. The study showed that in frozen food transportation, thermal emissions 
account for around $33 \%$ of total emissions. We also showed that considering temperature control can change the optimal speed. For ambient transportation, a speed around $40 \mathrm{~km} / \mathrm{h}$ results in the lowest emissions (Bektaş and Laporte, 2011), while for frozen food transportation, the optimal speed is around $60 \mathrm{~km} / \mathrm{h}$.

\section{Cooperative route planning and food quality}

In Chapter 3, we modelled food quality during cooperative transportation by extending a vehicle routing problem (VRP) with a kinetic model to describe quality decay on a multistop route. With this model, we showed that the estimation of the quality level of food during transportation can be improved and optimised by integrating kinetic modelling in routing models. This way, the effect of temperature fluctuations on a cooperative route can be considered. Extending the green VRP (Bektaş et al., 2016) with a quality decay minimisation-objective has shown that on a cooperative route, minimising quality decay can result in different optimal routes compared to minimising costs or emissions. Also, there is a significant difference in decay for the first partner that is visited on the route compared to the last one. Dependent on the type of products and their decay rate, this should be considered in route planning. For example by setting constraints on the maximum quality decay for each customer. However, this can result in increased costs and emissions.

\section{The effects of different forms of cooperation on eco-efficiency}

Chapter 4 provided a comparison between the eco-efficiency of a non-cooperative scenario to joint route planning (JRP) (Cruijssen et al., 2007b) and vendor-managed inventory (VMI) (Coelho et al., 2013) using an inventory routing problem (IRP) model, in which multiday planning and inventory are optimised (Cheng et al., 2017). JRP can provide significant benefits compared to individual routing, and VMI can provide even more savings, both in terms of costs and emissions. Other studies generally assume that reducing costs by cooperating can also result in emission savings. We found that ]RP cooperation results in the same optimal decisions independent of the objective of the model (i.e. cost or emission minimisation). However, in VMI, there is a trade-off between cost and emissions, and a set of alternative eco-efficient cooperative solutions exists. This implies that partners should agree on an eco-efficient solution that balances cost and emissions savings.

\section{Gain allocation for eco-efficient forms of logistics cooperation}

In Chapter 5, the Shapley value gain allocation method (Shapley, 1953) was used to allocate monetary gains based on contributions of the cooperating partners based on both costs and emission reductions. We demonstrated how the price of emissions can be calculated from an eco-efficient cooperation solution, and how that price can be used to allocate benefits using the Shapley value. For each eco-efficient solution benefits are distributed in a way that reflects the importance of economic and environmental goals of the cooperation. 


\subsection{Scientific impact}

The main research question (Which decision support models can be used to design eco-efficient logistics cooperation in food supply chains?) is answered by integrating the findings of the different chapters in four research themes: cooperative food logistics, cooperation and multiple objectives, eco-efficiency and gain allocation, and operations research (OR) models for cooperative food logistics.

\section{Cooperative food logistics}

In Chapter 2, 3 and 4, OR models were developed to evaluate the impact of cooperative food logistics on eco-efficiency. With these models, we showed that cooperation in food logistics can improve eco-efficiency by saving costs and emissions especially in temperature-controlled transportation, which is needed for fresh and frozen foods. Also, logistics cooperation can reduce quality decay. For quality decay minimisation however, other routes are optimal compared to cost or emission minimisation. Next to that, if multiple partners have high quality requirements, costs and emissions will increase. In order to become more eco-efficient using cooperation, partners should not minimise the total driving distance but their costs, emissions, or a combination of both. Fuel consumption should be considered in the estimation of costs and emissions. Fuel consumption depends on many aspects, but some of the most important ones are distance, load factor, order of unloading, driving speed, energy used for temperature control, and, in areas with altitude differences, the slopes of the road.

Companies in the food supply chain should also consider the effects of cooperation on food quality. On a cooperative route, the temperature fluctuations can cause products that are delivered later in the route to receive products with a lower quality. Moreover, when inventory and routing are optimised together, there exists a trade-off between product age and emissions which the cooperating partners should consider.

The effects of cooperative logistics on the eco-efficiency of food logistics depend on the form of cooperation. More advanced forms of cooperation, where more parts of the supply chain are executed together and more information is shared, can result in increased eco-efficiency of the supply chain. However, more advanced forms of cooperation result in more alternative eco-efficient solutions, which will increase complexity for partners: next to choosing a form of cooperation, they also need to choose between the alternative optimal solutions that different forms of cooperation provide.

\section{Cooperation and multiple objectives}

We have shown that logistics cooperation can reduce both cost and emissions, which is useful to convince potential partners to cooperate. Comparing different forms of cooperation has shown that joint route planning (JRP) and vendor-managed inventory (VMI) perform differently in terms of their eco-efficiency. VMI can provide further eco-efficiency improvements compared to JRP. However, in JRP there is one optimal solution that results in minimum costs and minimum emissions, while in VMI there is a set of alternative eco-efficient solutions. This means that partners need to choose which solution they 
prefer (Chapter 4). Other studies confirm that even if partner's objectives are conflicting, still forming a coalition can be beneficial for all partners (Defryn and Sörensen, 2018).

However, cooperation also requires streamlining of vehicles, routes, locations, products, and inventories (Chapter 3 and 4). Also there are multiple stakeholders, with possibly conflicting objectives. Indeed studies have shown that it is important to find a balance between efficiency improvement and an increase in complexity in cooperative logistics (Defryn and Sörensen, 2018). The advancement of new IT applications can be of help in dealing with this increased complexity (Kruize et al., 2016). For example, it is easier to monitor food quality with sensors that can track food quality throughout the supply chain (Akkerman et al., 2010).

\section{Eco-efficiency and gain allocation}

Fair gain allocation is an important prerequisite for long-term successful cooperation (Cruijssen et al., 2007a). In this thesis, gain allocation was based on both contributions of partners to saving costs and to saving emissions. This approach to gain allocation helps to stimulate eco-efficient cooperative solutions (Chapter 5). However, for partners to accept this form of gain allocation, a change of perspective might be needed. Saving emissions should be seen as a benefit instead of a burden. Indeed, studies have shown that the commitment of the upper management to climate change is an important predictor for the firm's climate change strategy, which may include carbon emission reduction plans (Eleftheriadis and Anagnostopoulou, 2017).

\section{OR models for cooperative food logistics}

To evaluate the eco-efficiency impacts of cooperation, operations research (OR) models can be used. In this thesis, OR models based on the VRP (Chapter 2 and 3 ) and the inventory routing problem (IRP) were used (Chapter 4 and 5). They were adjusted to account for the effects of temperature control and to quantify the effects of cooperation on food quality.

We have demonstrated the need to consider the effects of temperature control in fresh and frozen food transportation. Often, VRP and IRP models focus on reducing costs. Recently, green versions of VRP and IRP models have been developed to also consider emissions (Bektaş and Laporte, 2011; Cheng et al., 2017; Zachariadis et al., 2015). However, these type of models had not been adjusted to account for food specific issues. Therefore, we extended them to account for the costs and emissions related to temperature control, and to consider food quality as a consequence of cooperative routing. Moreover, in our IRP study, we calculated product age and found that less frequent inventory replenishment could save emissions but also increase average product age.

In the Introduction, a modelling framework was presented, and it was used to approach the main objective of this thesis: to develop decision support models that are able to capture the complexity of establishing effective long term food logistics cooperation in sup- 


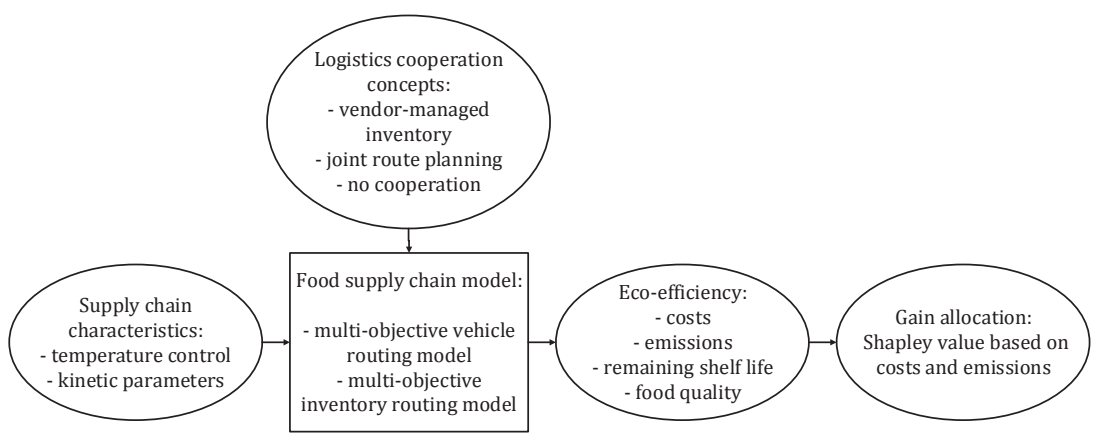

Figure 6.1: Modelling approach used in the thesis.

ply chains. In Chapters 2, 3, 4, and 5, different decision support models have been developed based on the modelling framework. Figure 6.1 shows the detailed version of the modelling framework, summarising the models and approaches used in this thesis.

Thus, VRP and IRP that are extended to account for temperature control and food quality can be used as decision support tools to design and test eco-efficient forms of logistics cooperation. However, dependent on the part of the supply chain and the scale of the cooperation, also other OR models could be of use. This thesis can be used as a guideline on how to adjust them to account for food specific aspects and cooperation.

\subsection{Societal impacts}

This thesis has focused on improving eco-efficiency, mostly by extending existing OR models with food aspects, and with an emission minimisation function. Cost minimisation is important for the company that has to pay the cost but emissions affect the whole society. Therefore, reducing emissions has more impact than reducing costs, from a societal perspective. We showed that temperature-controlled logistics has more environmental impact compared to ambient logistics. Therefore, in temperature-controlled logistics, it is even more important to improve eco-efficiency. Combined with the fact that food trucks often transport food towards and emit polluting gases in residential areas, there is a societal need to take emission reduction action in temperature-controlled transportation, and logistics cooperation is a way to do so.

Gain allocation is an important prerequisite for a successful cooperation. When eco-efficient cooperation works on the long term, emissions are saved, which benefits the society. Instead of studying how to save costs or emissions, we studied how to save and reward both. In society, for example when discussing taxes on emissions, it is often implicitly assumed that saving emissions comes at some costs. This research shows that saving costs and saving emissions can go hand in hand. 
We focused our study on eco-efficiency, by considering costs as economic indicator and emissions as environmental indicator. We also considered food quality, which could be considered both an economic and an environmental indicator, since quality affects price, but also food waste. Improving costs, emissions and food quality are thus all part of an eco-efficient food supply chain. Improving eco-efficiency can be seen as a first step towards improving sustainability (Huppes and Ishikawa, 2005). Besides the environmental and economic dimensions, sustainability also has a social dimension that we did not study in this thesis. This is still an important future research direction.

\subsection{Managerial insights}

\section{The CapsLog project}

This PhD thesis is part of the CapsLog project, which is funded by the NWO (Netherlands Organisation for Scientific Research). CapsLog stands for Capitalising on cooperation in sustainable logistics in food chains. The case studies used in this thesis originate from a group of Dutch retail companies that cooperatively buy products, but they were interested in also cooperative organising logistics. The study results were communicated with the retail organisation, and they have implemented eco-efficiency improving measures inspired by the studies in this thesis. Moreover, a supply chain advisory was involved. Before the project, they were mainly focused on minimising distance, but they are now starting to advice other companies to implement cooperative cost and emission minimising solutions.

\section{Starting logistics cooperation}

Decision makers in food logistics are confronted with the challenge to make their companies more eco-efficient and cooperation is one way to do that. However, food logistics, cooperation, eco-efficiency and gain sharing are complex topics. In many studies, cooperation has been qualitatively assessed, and quantitative assessments have often only focused on one form of cooperation and on monetary benefits. Since this thesis analyses the effects of cooperation on both costs and emissions, and different forms of cooperation are compared, it will help decision makers in getting more insight in the ecoefficiency effects of different forms of cooperation, such that they can make a more informed decision on cooperation.

\section{Effects of logistics cooperation}

In this thesis, analyses were done to test the effect of different parameters on costs and emissions. To quantify costs, emissions, and eco-efficiency correctly, it is important to have a precise fuel estimation, and (in the case of fresh and frozen food products) to consider temperature control. Especially in areas with hills or mountains, considering the slope of the road in the route planning can result in significant fuel savings as well. Also, we confirmed the importance of considering the weight of the load and the order of unloading for fuel consumption, and the resulting costs and emissions. We also studied the effect of cooperation on food quality. For managers, it can be reassuring to know that cooperation does not always yield products of lower quality. However, on a cooperative 
route with highly perishable goods and long door opening times, partners that are visited later in the route do get products with a lower quality compared to partners that are visited earlier in a route. Partners that decide to organise cooperative routes with perishable products together should thus also think about this.

\section{Maintaining logistics cooperation}

Fair gain allocation is important to make cooperation work in the long term. When decision makers decide to work together, emission reduction is often seen as a reason to cooperate (Makaci et al., 2017). The gain allocation study can be of help for partners that want to reward both efforts to reduce costs and to reduce emissions, such that the cooperation also leads to emission reductions.

\subsection{Validity of the findings}

Here, we discuss the validity of the findings related to case study choices, modelling choices, and regulatory aspects.

\section{Case study}

All studies in this thesis are based on a case study on a group of retail organisations in The Netherlands. Since we used the same case study for the different studies, it allows for easy comparison of the results within this thesis. However different case studies would allow for easier generalisation. On the other hand, the case study is a stylised example, which allows for a more general interpretation, and for interested companies to test the findings with their data. Our case study was based a data set that was small enough to be solved exactly but large enough to show relations between important parameters. When applying the methods described in this thesis in practise, data sets will be bigger and heuristics will need to be used. There has been a significant amount of research on heuristics for (green) VRPs and IRPs, such as simulated annealing (Xiao and Konak, 2015) and variable neighbourhood search (Jabir et al., 2017).

In most of the studies in this thesis, temperature-controlled transportation is assumed. The data related to calculating the effects of temperature control are based on a limited number of sources, which makes it hard to validate them. This implies that more research on temperature-controlled transportation should be done. For simplification and because of a lack of data, the self-insulating effect of the cooled load was not considered. It is expected that this effect does play a role in reducing the thermal energy needed to cool the load, especially in frozen food transportation.

In our case study, there was already a shared distribution centre. In other forms of cooperation, it could be needed to start a consolidation centre, which brings advantages and barriers for the cooperation (Paddeu et al., 2018). In our study, cooperation mainly resulted in financial benefits that needed to be divided fairly. However, when starting a consolidation centre, there are costs that need to be shared, especially in the beginning. Since most people are loss averse, the prospect of first having to pay in order to gain later in the cooperation is probably not attractive. This is why it is important that we quantified the benefits of cooperation; that can help convincing potential partners to invest in 
cooperation.

\section{Optimisation models}

We have shown possibilities for supply chain improvements using logistics cooperation. In the real world however, logistics decisions are made by people. More and more, people use (optimisation or simulation) computer models to support their decisions. But still, in order to change towards a more efficient and sustainable way of working, people need to implement changes. In case of implementing cooperation, this is even harder as multiple people need to work together, and an improvement for the overall supply chain could come at the cost of harming one part of the supply chain. Cooperation should create enough value for the partners such that they make sure it endures (Morgan and Hunt, 1994). At the same time, there exist barriers that can prevent partners from fully cooperating and reaching the cooperation objectives (Fawcett et al., 2015). These kind of issues cannot be solved by optimisation models; model findings should always be combined with a managerial approach in order to implement them in practise (Badraoui et al., 2019).

\section{Regulatory aspects}

We quantified benefits and drawbacks of cooperation in terms of costs, emissions, food quality, and we studied how cooperative gains could be shared. However, we did not discuss regulatory aspects since it is outside our scope and outside our area of expertise. Nonetheless, it should be mentioned that the antitrust law can complicate cooperative settings, and cooperation will result in an administrative burden for the partners. The antitrust law forbids forms of cooperation that harm the consumer. For example, price agreements are a form of cooperation that is not allowed by the antitrust law. Since logistics cooperation does not directly affect consumer prices, we do not expect that it causes difficulties in that sense. Logistics cooperation could even result in lower consumer prices.

\subsection{Future research directions}

\section{Eco-efficiency and supply chain resilience}

To remain competitive and satisfy the increasing demand for sustainable products, food supply chains must adapt. Cooperation is proven to be a logistical concept which can bring economic benefits to the companies of the supply chain involved but also environmental benefits for the society. However, being $100 \%$ efficient should not be an aim, because then supply chain resilience can be compromised. Supply chain resilience is the capability of a supply chain to deal with unexpected deviations from the norm and their negative consequences (Pettit et al., 2010). On the other hand, Scholten and Schilder (2015) show that supply chain cooperation also provides opportunities to improve resilience. Their study focused on buyer-supplier cooperation, while this thesis also studied horizontal cooperation; between two partners at the same level in the supply chain. Finding the balance between eco-efficiency and resilience could be a topic for further research. 


\section{Larger scale case studies}

The case study was big enough to show relations between important parameters of the model and results of the analysis, but small enough to solve exactly. For bigger cases (e.g. with more partners or a bigger part of the supply chain), bigger data sets will be used and heuristics and approximation methods are needed (Arnold et al., 2017). Gain allocation methods such as the Shapley value have been approximated (Fatima et al., 2008), which could be used in future research with bigger case studies. Moreover, different allocation methods could be used for bigger case studies (Frisk et al., 2010).

Also, the introduction of this thesis gave a limited overview of different forms of logistics cooperation. In this thesis, JRP and VMI cooperation were compared to a non-cooperative scenario. However, a classification of more forms of cooperation could be used to compare and quantify the effects of more different forms of cooperation (Badraouiet al., 2019).

\section{Applied gain allocation studies}

In this thesis, the Shapley value gain allocation method was applied in a new way, to account for economic and environmental contributions of cooperating partners. The approach to allocate benefits based on costs and emissions could also be combined with other gain allocation methods. Future research directions based on gain allocation methods could also focus on finding fair allocations of product quality on cooperative routes. Also, the acceptability of different gain allocation methods could be tested in practise.

\section{Environmental indicators}

In this thesis, $\mathrm{CO}_{2}$ emissions were quantified as measure for environmental impact. However, road transportation causes many more types of emissions, which can be divided in greenhouse gases and local pollutants. Greenhouse gases have a large scale effect and are linked to the greenhouse effect, which causes global warming. Of the different greenhouse gasses, $\mathrm{CO}_{2}$ is estimated to account for two thirds of the global warming effect. Local pollutants exhausted by vehicle engines are carbon monoxide (CO), nitrogen oxides (NOx), fine particles, and hydrocarbons. Local pollutants from vehicle engines remain in the area in which they are exhausted (Palmer, 2007). For a more comprehensive view of the environmental effects of cooperative logistics, it would be good to test the effects on more types of emissions. For local pollutants, legislation is in place to limit these emissions (Palmer, 2007). Recently, international agreements, such as the Paris agreement have been made to limit greenhouse gases. However, studies mention that the plans are not strict enough to contain climate change, and the United States have announced to step out of the agreement (Rogelj et al., 2016). Therefore, it is important to keep studying the causes of climate change such that measures can be taken to mitigate its effects. This study has shown that logistics cooperation is an effective way to reduce emissions, so policy makers that want to reduce emissions should consider stimulating logistics cooperation. Also, we have shown that reducing emissions can also result in cost reductions. This finding can help convince companies to implement emission reduction measures such as cooperative logistics. 


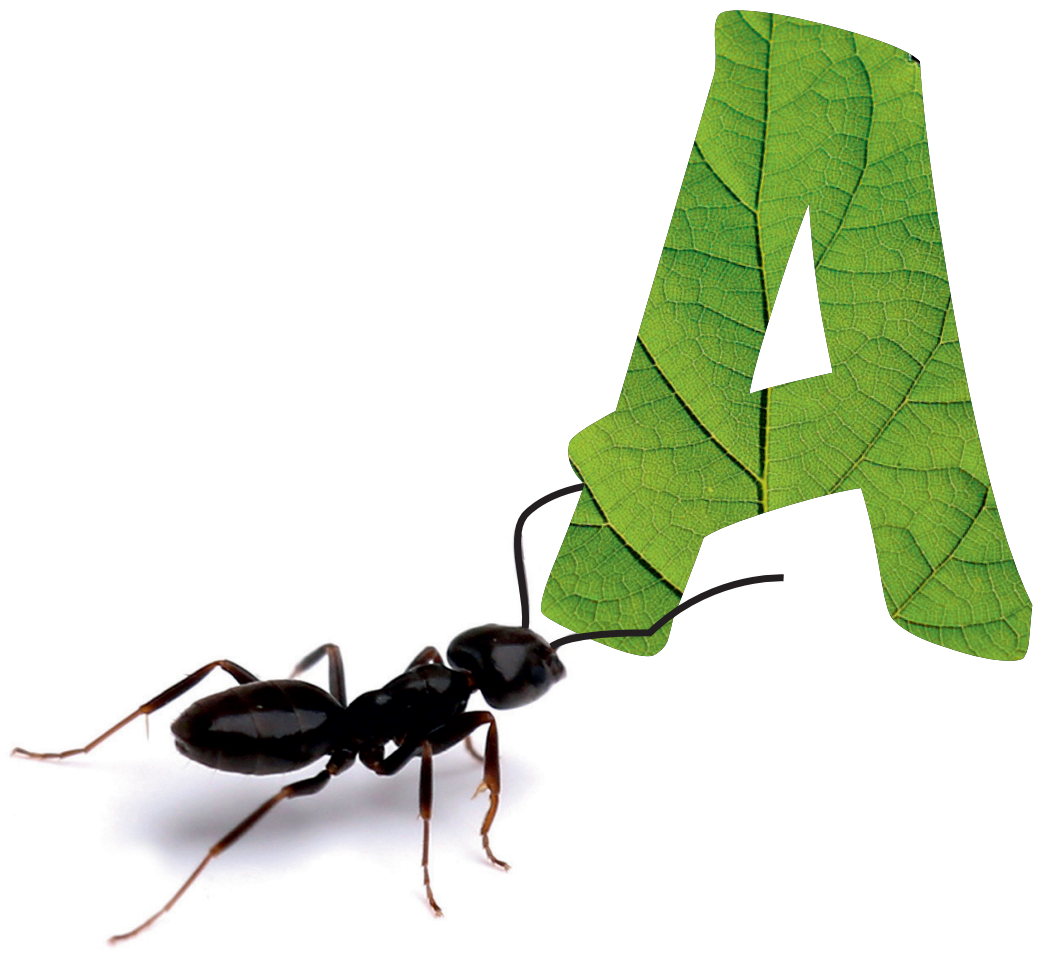




\section{Appendix}

Table A.1: Clossary.

\begin{tabular}{ll}
\hline Abbreviation & Explanation \\
\hline CDC & Central Distribution Center \\
CGT & Cooperative Game Theory \\
$\mathrm{CO}_{2}$ & Carbon dioxide \\
$\mathrm{COP}$ & Coefficient of Performance \\
DC & Distribution Center \\
CVRP & Creen Vehicle Routing Problem \\
HDV & Heavy Duty Vehicle \\
IRP & Inventory Routing Problem \\
JRP & Joint Route Planning \\
LDV & Light Duty Vehicle \\
LDVRP & Load Dependent Vehicle Routing Problem \\
MDV & Medium Duty Vehicle \\
MILP & Mixed Integer Linear Programming \\
VMI & Vendor Managed Inventory \\
VRP & Vehicle routing problem \\
OR & Operations Research \\
TCLDVRP & Temperature Controlled Load Dependent \\
& Vehicle Routing Problem \\
\hline
\end{tabular}

Table A.2: Decision variables used in the chapters.

\begin{tabular}{|c|c|c|c|}
\hline $\begin{array}{c}\text { Decision } \\
\text { variables }\end{array}$ & unit & $\begin{array}{l}\text { used in } \\
\text { chapter }\end{array}$ & definition \\
\hline$D_{i j}^{k p}$ & - & 3 & decay at an arc \\
\hline$D_{i j, v}^{k j}$ & - & 3 & decay during cooling time (at a variable temperature) at an arc \\
\hline$D_{i j, f}^{k p,}$ & - & 3 & decay at a fixed temperature on an arc \\
\hline$I_{i r}^{t}$ & $\mathrm{~kg}$ & 4 & inventory at DC $i$ of age $r$ in period $t$ \\
\hline$q_{i r}^{t}$ & $\mathrm{~kg}$ & 4 & demand fulfilled from DC $i$ of age $r$ in period $t$ \\
\hline$Q_{i j}^{p m}$ & - & 3 & quality level arriving at node $j$ from node $i$ of product $p$ with vehicle $k$ \\
\hline$s c_{i j}^{k}$ & s & 3 & cooling time of vehicle $k$ on arc $i j$ \\
\hline$u_{i}$ & - & 2 & index for order number in a delivery route (MTZ constraints) \\
\hline$x_{i j}^{k}$ & 0,1 & $2,3,4,5$ & binary: 1 if arc $i j$ is crossed with vehicle $k, 0$ otherwise \\
\hline$w_{i j}^{k}$ & $\mathrm{~kg}$ & 2 & weight transported on vehicle $k$ from node $i$ to node $j$ (incl. vehicle weight) \\
\hline$w_{i j}^{k p}$ & $\mathrm{~kg}$ & 3 & weight product $p$ transported on vehicle $k$ from node $i$ to node $j$ (ex. vehicle weight) \\
\hline$w_{i j}^{k t}$ & $\mathrm{~kg}$ & 4 & weight transported on vehicle $k$ from node $i$ to node $j$ in period $t$ (incl. vehicle weight) \\
\hline$y_{i}^{k t}$ & $\mathrm{~kg}$ & 4 & quantity delivered to DC $i$ by vehicle $k$ in period $t$ \\
\hline$z_{i j}^{k}$ & 0,1 & 3 & binary for big $\mathrm{M}$ method \\
\hline$Z$ & - & 3 & variable to minimise maximum quality decay \\
\hline
\end{tabular}


Table A.3: Values used to run the models in the different chapters.

\begin{tabular}{|c|c|c|c|c|c|}
\hline Symbol & Value & Unit & in chapter & Definition & Source \\
\hline$\alpha_{i j}$ & 0.0981 & $\mathrm{~m} / \mathrm{s}^{2}$ & $2,3,4,5$ & arc-specific constant & Bektaş and Laporte (2011) \\
\hline$\beta$ & 3.013 & $\mathrm{~kg} / \mathrm{m}$ & $2,3,4,5$ & vehicle-specific constant & Bektaş and Laporte (2011) \\
\hline$\Delta T$ & $0,8,18,28,38,48$ & $\mathrm{~K}$ & $2,3,4,5$ & Temperature difference vehicle and outside & Akkermanetal. (2010) \\
\hline$\eta_{e}$ & 0.3 & - & $2,3,4,5$ & efficiency chemical to refrigeration energy & Bell (2008) \\
\hline$\eta_{m}$ & 0.3 & - & $2,3,4,5$ & motive energy conversion efficiency & Bektaş and Laporte (2011) \\
\hline$\rho$ & 1.2041 & $\mathrm{~kg} / \mathrm{m}^{3}$ & $2,3,4,5$ & air density & Bektaşand Laporte (2011) \\
\hline$A$ & 7.15 & $\mathrm{~m}^{2}$ & $2,3,4,5$ & frontal area of the vehicle & Bektaş and Laporte (2011) \\
\hline$c_{0}$ & $0.0005,0.001,0.0015$ & $€ / \mathrm{kg}$ & 4 & unit inventory cost CDC & Soysal et al. (2015) \\
\hline$C_{d}$ & 0.7 & - & $2,3,4,5$ & coefficient of drag & Bektaş and Laporte (2011) \\
\hline$c_{f}$ & 1.4 & $€ / L$ & $2,3,4,5$ & unit fuel cost & Bektaşand Laporte (2011) \\
\hline$c_{I}$ & $0.005,0.01,0.015$ & $€ / \mathrm{kg}$ & 4 & unit inventory cost $D C$ & Soysal et al. (2015) \\
\hline$C_{r}$ & 0.01 & - & $2,3,4,5$ & rolling resistance & Bektaş and Laporte (2011) \\
\hline$c_{w}$ & 0.0022 & $€ / s$ & $2,3,4,5$ & unit wage cost & Bektaş and Laporte (2011) \\
\hline$C O P$ & $0.67,2$ & - & $2,3,4,5$ & coefficient of performance & Bektaşet al. (2016) \\
\hline$e_{f}$ & 2.668 & $\mathrm{~kg} / \mathrm{L}$ & $2,3,4,5$ & fuel to $\mathrm{CO}_{2}$ emissions factor & Tassou et al. (2009) \\
\hline$e_{r}$ & 1.205 & $\mathrm{~kg} / \mathrm{kg}$ & $2,3,4,5$ & emission factor refrigerated transport & Koehleret al. (1997) \\
\hline$g$ & 9.81 & $\mathrm{~m} / \mathrm{s}^{2}$ & $2,3,4,5$ & gravitation constant & Koehler et al. (1997) \\
\hline$h_{i}$ & 4 & $\mathrm{kWh}$ & $2,4,5$ & heat entering during service time at stop $i$ & Tso et al. (2002) \\
\hline$k$ & $3,4,7$ & & $2,3,4,5$ & number of vehicles & Tso et al. (2002) \\
\hline$P_{f}$ & 8.8 & $\mathrm{kWh} / \mathrm{L}$ & $2,3,4,5$ & energy content of the fuel & Bektaşand Laporte (2011) \\
\hline$L$ & $7500,12500,30000$ & $\mathrm{~kg}$ & $2,3,4,5$ & truck capacity & Bektaş and Laporte (2011) \\
\hline$L_{0}$ & $4500,5500,10000$ & $\mathrm{~kg}$ & $2,3,4,5$ & curb weight & Koçetal. (2016) \\
\hline$s_{i}$ & 900 & $s$ & $2,4,5$ & service time at each stop & Koçet al. (2016) \\
\hline$S^{k}$ & 165 & $\mathrm{~m}^{2}$ & $2,3,4,5$ & the surface area of vehicle $k$ & Tassou et al. (2009) \\
\hline$U$ & 0.7 & $\mathrm{~W} / \mathrm{m}^{2} / \mathrm{K}$ & $2,3,4,5$ & heat transfer coefficient & Tassou et al. (2009) \\
\hline
\end{tabular}


Table A.4: Parameters used in models.

\begin{tabular}{|c|c|c|}
\hline Symbol & Unit & Definition \\
\hline$\alpha_{i j}$ & $\mathrm{~m} / \mathrm{s}^{2}$ & arc-specific constant \\
\hline$\beta$ & $\mathrm{kg} / \mathrm{m}$ & vehicle-specific constant \\
\hline$\Delta T$ & K & difference in temperature inside an outside vehicle \\
\hline$\eta_{e}$ & - & chemical to refrigeration energy conversion efficiency \\
\hline$\eta_{m}$ & - & motive energy conversion efficiency \\
\hline$\eta_{p}$ & - & coefficient of performance \\
\hline$\lambda$ & $€ / \mathrm{kg}$ & price of $\mathrm{CO}_{2}$ emissions \\
\hline$\rho$ & $\mathrm{kg} / \mathrm{m}^{3}$ & air density \\
\hline$\theta_{i j}$ & & slope of the road \\
\hline$a$ & $\mathrm{~m} / \mathrm{s}^{2}$ & acceleration of the vehicle \\
\hline$A$ & $\mathrm{~m}^{2}$ & frontal area of the vehicle \\
\hline$C$ & $€$ & total cost \\
\hline$c_{i j}$ & $\mathrm{~m}$ & distance of arc $i j$ \\
\hline$c_{0}$ & $€ / \mathrm{kg}$ & unit inventory cost $C D C$ \\
\hline$C_{d}$ & - & coefficient of drag \\
\hline$c_{f}$ & $€ / L$ & unit fuel cost \\
\hline$c_{I}$ & $€ / \mathrm{kg}$ & unit inventory cost DC \\
\hline$C_{I}$ & $€$ & inventory cost \\
\hline$C_{i}$ & $\mathrm{~kg}$ & capacity DC $i$ \\
\hline$c_{p, a}$ & $\mathrm{~J} /\left(\mathrm{m}^{3} \mathrm{~K}\right)$ & volumetric heat capacity \\
\hline$C_{r}$ & - & rolling resistance \\
\hline$C_{t}$ & $€$ & transportation cost \\
\hline$c_{w}$ & $€ / \mathrm{s}$ & unit wage cost \\
\hline$d$ & s & maximum driving time \\
\hline$E$ & $\mathrm{kgCO}_{2}$ & total emissions for transportation \\
\hline$E_{n}$ & $\mathrm{kgCO}_{2}$ & emissions of non-refrigerated transport \\
\hline$E_{a}^{p}$ & Joule/mol & activation energy of product $p$ \\
\hline$e_{f}^{a}$ & $\mathrm{~kg} / \mathrm{L}$ & fuel to $\mathrm{CO}_{2}$ emissions factor \\
\hline$E_{r}$ & $\mathrm{kgCO}_{2}$ & emissions of refrigerated transport \\
\hline$e_{r}$ & $\mathrm{~kg} / \mathrm{kg}$ & emission factor refrigerated transport \\
\hline$f_{a}$ & L & fuel use for ambient transport \\
\hline$f_{r}$ & L & fuel use for refrigerated transport \\
\hline$f$ & $\mathrm{~K} / \mathrm{s}$ & temperature increase speed \\
\hline$g$ & $\mathrm{~m} / \mathrm{s}^{2}$ & gravitation constant \\
\hline$h_{i}$ & $\mathrm{kWh}$ & heat entering during service time at stop $i$ \\
\hline$H_{s}$ & $\mathrm{kWh}$ & heat entering during service time \\
\hline$H_{w}$ & $\mathrm{kWh}$ & heat entering through the wall \\
\hline$\kappa$ & $\mathrm{mol} /(\mathrm{Ls})$ & rate constant \\
\hline $\begin{array}{l}\kappa_{0} \\
k\end{array}$ & $\mathrm{~mol} /(\mathrm{Ls})$ & $\begin{array}{l}\text { pre-exponential factor } \\
\text { index for vehicles }\end{array}$ \\
\hline$L^{k}$ & $\mathrm{~kg}$ & capacity of vehicle $k$ \\
\hline$L_{0}$ & $\mathrm{~kg}$ & curb weight \\
\hline$M$ & - & big number \\
\hline$P_{i j}$ & $\mathrm{kWh}$ & motive power on arc $i j$ \\
\hline$P_{f}$ & $\mathrm{kWh} / \mathrm{L}$ & energy content of the fuel \\
\hline$q_{i}^{p p}$ & - & demand node $i$ of product $p$ \\
\hline$Q_{0}^{p}$ & - & initial quality product $p$ \\
\hline$R$ & $\mathrm{~J} /(\mathrm{mol} \mathrm{K})$ & gas constant \\
\hline $\bar{r}$ & days & average product age \\
\hline$s_{i}$ & s & service time at stop $i$ \\
\hline$S^{k}$ & $\mathrm{~m}^{2}$ & the surface area of vehicle $k$ \\
\hline$s c_{i j}^{k}$ & s & cooling time of vehicle $k$ on arc $i j$ \\
\hline$T^{i J}$ & K & temperature in Kelvin \\
\hline$T_{0}$ & K & goal temperature \\
\hline$T_{0}^{k}$ & $\mathrm{~K}$ & air temperature in vehicle $k$ when it leaves the $C D C$ \\
\hline$T_{a}$ & K & ambient temperature \\
\hline$T_{i}^{k}$ & $\mathrm{~K}$ & air temperature of vehicle $k$ at the end of the service time at node $i$ \\
\hline$T_{i}^{k p}$ & $\mathrm{~K}$ & air temperature of vehicle $k$ at the end of the service time at node $i$ \\
\hline$T_{r e f}^{p}$ & $\mathrm{~K}$ & reference (optimal) temperature of product $p$ \\
\hline$t_{s}$ & $\mathrm{~s} / \mathrm{kg}$ & unloading rate \\
\hline$U$ & $\mathrm{~W} / \mathrm{m}^{2} / \mathrm{K}$ & heat transfer coefficient \\
\hline$v_{i j}$ & $\mathrm{~m} / \mathrm{s}$ & speed driven on arc $i j$ \\
\hline$V_{k}$ & $\mathrm{~m}^{3}$ & volume of vehicle $k$ \\
\hline$y_{j}^{c}$ & $€$ & cost allocated to partner $j$ \\
\hline$y_{j}^{e}$ & $\mathrm{kgCO}_{2}$ & emissions allocated to partner $j$ \\
\hline$y_{j}^{K}$ & - & K-value allocated to partner $j$ \\
\hline
\end{tabular}


Table A.5: Base case speed matrix $(\mathrm{km} / \mathrm{h})$ used in Chapter 3.

\begin{tabular}{r|rrrrrrrr} 
& 0 & 1 & 2 & 3 & 4 & 5 & 6 & 7 \\
\hline 0 & & 45.9 & 50.7 & 51.4 & 63.4 & 65.9 & 50.5 & 46.7 \\
1 & 59.1 & & 42.9 & 48.1 & 60.2 & 65.3 & 44.5 & 46.9 \\
2 & 64.2 & 60.3 & & 60.2 & 66.4 & 63.6 & 59.1 & 50.5 \\
3 & 61.0 & 64.7 & 44.2 & & 67.9 & 58.9 & 51.3 & 52.3 \\
4 & 41.8 & 49.7 & 50.0 & 50.4 & & 65.5 & 48.2 & 48.1 \\
5 & 50.7 & 52.1 & 46.8 & 47.8 & 42.3 & & 42.5 & 50.2 \\
6 & 62.5 & 63.1 & 44.9 & 65.3 & 65.2 & 61.1 & & 50.7 \\
7 & 62.8 & 56.8 & 59.7 & 59.8 & 59.3 & 59.2 & 60.9 & \\
\hline
\end{tabular}

Table A.6: Demand and location data used for the demand and distance sensitivity analysis instances in Chapter 4, based on the Solomon R101 data set (Solomon, 1987). The DC number refers to the day in the Solomon data set of which the $x$ and $y$ coordination were taken.

\begin{tabular}{|c|c|c|c|c|c|c|c|}
\hline Data set & $\mathrm{DC}$ & Monday & Tuesday & Wednesday & Thursday & Friday & Saturday \\
\hline \multirow[t]{7}{*}{1} & 1 & 1 & 8 & 15 & 22 & 29 & 36 \\
\hline & 2 & 2 & 9 & 16 & 23 & 30 & 37 \\
\hline & 3 & 3 & 10 & 17 & 24 & 31 & 38 \\
\hline & 4 & 4 & 11 & 18 & 25 & 32 & 39 \\
\hline & 5 & 5 & 12 & 19 & 26 & 33 & 40 \\
\hline & 6 & 6 & 13 & 20 & 27 & 34 & 41 \\
\hline & 7 & 7 & 14 & 21 & 28 & 35 & 42 \\
\hline \multirow[t]{7}{*}{2} & 8 & 1 & 8 & 15 & 22 & 29 & 36 \\
\hline & 9 & 2 & 9 & 16 & 23 & 30 & 37 \\
\hline & 10 & 3 & 10 & 17 & 24 & 31 & 38 \\
\hline & 11 & 4 & 11 & 18 & 25 & 32 & 39 \\
\hline & 12 & 5 & 12 & 19 & 26 & 33 & 40 \\
\hline & 13 & 6 & 13 & 20 & 27 & 34 & 41 \\
\hline & 14 & 7 & 14 & 21 & 28 & 35 & 42 \\
\hline \multirow[t]{7}{*}{3} & 15 & 1 & 8 & 15 & 22 & 29 & 36 \\
\hline & 16 & 2 & 9 & 16 & 23 & 30 & 37 \\
\hline & 17 & 3 & 10 & 17 & 24 & 31 & 38 \\
\hline & 18 & 4 & 11 & 18 & 25 & 32 & 39 \\
\hline & 19 & 5 & 12 & 19 & 26 & 33 & 40 \\
\hline & 20 & 6 & 13 & 20 & 27 & 34 & 41 \\
\hline & 21 & 7 & 14 & 21 & 28 & 35 & 42 \\
\hline \multirow[t]{7}{*}{4} & 22 & 1 & 8 & 15 & 22 & 29 & 36 \\
\hline & 23 & 2 & 9 & 16 & 23 & 30 & 37 \\
\hline & 24 & 3 & 10 & 17 & 24 & 31 & 38 \\
\hline & 25 & 4 & 11 & 18 & 25 & 32 & 39 \\
\hline & 26 & 5 & 12 & 19 & 26 & 33 & 40 \\
\hline & 27 & 6 & 13 & 20 & 27 & 34 & 41 \\
\hline & 28 & 7 & 14 & 21 & 28 & 35 & 42 \\
\hline \multirow[t]{7}{*}{5} & 29 & 1 & 8 & 15 & 22 & 29 & 36 \\
\hline & 30 & 2 & 9 & 16 & 23 & 30 & 37 \\
\hline & 31 & 3 & 10 & 17 & 24 & 31 & 38 \\
\hline & 32 & 4 & 11 & 18 & 25 & 32 & 39 \\
\hline & 33 & 5 & 12 & 19 & 26 & 33 & 40 \\
\hline & 34 & 6 & 13 & 20 & 27 & 34 & 41 \\
\hline & 35 & 7 & 14 & 21 & 28 & 35 & 42 \\
\hline
\end{tabular}


Appendix 



\section{Bibliography}

Adekomaya, O., T. Jamiru, R. Sadiku, and Z. Huan (2016), Sustaining the shelf life of fresh food in cold chain-a burden on the environment, Alexandria Engineering Journal, 55(2), 1359-1365.

Adenso-Díaz, B., S. Lozano, S. Garcia-Carbajal, and K. Smith-Miles (2014), Assessing partnership savings in horizontal cooperation by planning linked deliveries, Transportation Research Part A: Policy and Practice, 66, 268279.

Ahn, K., and H. Rakha (2008), The effects of route choice decisions on vehicle energy consumption and emissions, Transportation Research Part D: Transport and Environment, 13(3), 151-167.

Akkerman, R., P. Farahani, and M. Crunow (2010), Quality, safety and sustainability in food distribution: a review of quantitative operations management approaches and challenges, OR Spectrum, 32(4), 863-904.

Ambrosino, D., and A. Sciomachen (2007), A food distribution network problem: a case study, IMA Journal of Management Mathematics, 18(1), 33-53.

Amorim, P., and B. Almada-Lobo (2014), The impact of food perishability issues in the vehicle routing problem, Computers \& Industrial Engineering, 67, 223-233.

Arnold, F., M. Gendreau, and K. Sörensen (2017), Efficiently Solving Very Large Scale Routing Problems, CIRRELT.

Aung, M. M., and Y. S. Chang (2014), Temperature management for the quality assurance of a perishable food supply chain, Food Control, 40, 198-207.

Aydin, G., and E. L. Porteus (2008), Joint inventory and pricing decisions for an assortment, Operations Research, 56(5), 1247-1255.

Aziz, H. A., and S. V. Ukkusuri (2014), Exploring the trade-off between greenhouse gas emissions and travel time in daily travel decisions: Route and departure time choices, Transportation Research Part D: Transport and Environment, 32, 334-353.

Badraoui, I., I. Van der Lans, Y. Boulaksil, and J. C. Van der Vorst (2019), Intentions versus actual actions in horizontal logistics collaboration, Journal of Operations and Production Management, (submitted).

Banasik, A., J. M. Bloemhof-Ruwaard, A. Kanellopoulos, G. D. H. Claassen, and J. G. A. J. van der Vorst (2016), Multi-criteria decision making approaches for green supply chains: a review, Flexible Services and Manufacturing Journal, pp. 1-31.

Banasik, A., J. M. Bloemhof-Ruwaard, A. Kanellopoulos, G. Claassen, and]. G. van der Vorst (2018), Multi-criteria decision making approaches for green supply chains: A review, Flexible Services and Manufacturing Journal, 30(3), 366-396.

Barth, M., and K. Boriboonsomsin (2009), Energy and emissions impacts of a freeway-based dynamic ecodriving system, Transportation Research Part D: Transport and Environment, 14(6), 400-410.

Bazan, E., M. Y. Jaber, and S. Zanoni (2015), Supply chain models with greenhouse gases emissions, energy usage and different coordination decisions, Applied Mathematical Modelling, 39(17), 5131-5151.

Bektaş, T., E. Demir, and G. Laporte (2016), Green vehicle routing, in Green Transportation Logistics, pp. 243-265, Springer.

Bektaş, T., and G. Laporte (2011), The pollution-routing problem, Transportation Research Part B: Methodological, 45(8), 1232-1250.

Bell, L. E. (2008), Cooling, heating, generating power, and recovering waste heat with thermoelectric systems, Science, 321(5895), 1457-1461. 
Bing, X., M. de Keizer, J. M. Bloemhof-Ruwaard, and J. G. van der Vorst (2014), Vehicle routing for the ecoefficient collection of household plastic waste, Waste management, 34(4), 719-729.

Bloemhof, J. M., J. G. A. J. Van der Vorst, M. Bastl, and H. Allaoui (2015), Sustainability assessment of food chain logistics, International Journal of Logistics Research and Applications, 18(2), 101-117.

Borade, A. B., and E. Sweeney (2015), Decision support system for vendor managed inventory supply chain: a case study, International Journal of Production Research, 53(16), 4789-4818.

Bozorgi, A. (2016), Multi-product inventory model for cold items with cost and emission consideration, International Journal of Production Economics, 176, 123-142.

Bozorgi, A., J. Pazour, and D. Nazzal (2014), A new inventory model for cold items that considers costs and emissions, International Journal of Production Economics, 155, 114-125.

Cai, J., X. Hu, P. R. Tadikamalla, and ]. Shang (2017), Flexible contract design for VMI supply chain with servicesensitive demand: Revenue-sharing and supplier subsidy, European Journal of Operational Research, 261(1), 143-153.

CBS (2018), CO 2 -prijs emissiehandel, last accessed november 2018.

Chakraborty, A., A. K. Chatterjee, and A. Mateen (2015), A vendor-managed inventory scheme as a supply chain coordination mechanism, International Journal of Production Research, 53(1), 13-24.

Chen, H.-K., C.-F. Hsueh, and M.-S. Chang (2009), Production scheduling and vehicle routing with time windows for perishable food products, Computers \& Operations Research, 36(7), 2311-2319.

Chen, L., X. Zhao, O. Tang, L. Price, S. Zhang, and W. Zhu (2017), Supply chain collaboration for sustainability: A literature review and future research agenda, International Journal of Production Economics, 194, $73-87$.

Cheng, C., P. Yang, M. Qi, and L.-M. Rousseau (2017), Modeling a green inventory routing problem with a heterogeneous fleet, Transportation Research Part E: Logistics and Transportation Review, 97, 97-112.

Chopra, S., and P. Meindl (2007), Supply chain management. strategy, planning \& operation, in Das summa summarum des management, pp. 265-275, Springer.

Coelho, L. C., J.-F. Cordeau, and G. Laporte (2013), Thirty years of inventory routing, Transportation Science, 48(1), 1-19.

Cruijssen, F. C. A. M. (2012), Framework for collaboration: A CO 3 position paper, Collaboration Concepts for Comodality.

Cruijssen, F. C. A. M., W. E. H. Dullaert, and H. A. Fleuren (2007a), Horizontal cooperation in transport and logistics: a literature review, Transportation Journal, 46(3), 22-39.

Cruijssen, F. C. A. M., O. Bräysy, W. E. H. Dullaert, H. A. Fleuren, and M. Salomon (2007b), Joint route planning under varying market conditions, International Journal of Physical Distribution \& Logistics Management, 37(4), 287-304.

Cruijssen, F. C. A. M., M. Cools, and W. E. H. Dullaert (2007c), Horizontal cooperation in logistics: opportunities and impediments, Transportation Research Part E: Logistics and Transportation Review, 43(2), 129-142.

Cruijssen, F. C. A. M., P. E. M. Borm, H. A. Fleuren, and H. J. M. Hamers (2010), Supplier-initiated outsourcing: A methodology to exploit synergy in transportation, European Journal of Operational Research, 207(2), 763-774.

Defryn, C., and K. Sörensen (2018), Multi-objective optimisation models for the travelling salesman problem with horizontal cooperation, European Journal of Operational Research, 267(3), 891-903.

Defryn, C., C. Vanovermeire, and K. Sörensen (2016), Gain sharing in horizontal logistic co-operation: a case study in the fresh fruit and vegetables sector, in Lu M., De Bock J. (eds) Sustainable Logistics and Supply Chains, pp. 75-89, Springer, Cham.

Dekker, R., J. M. Bloemhof, and I. Mallidis (2012), Operations research for green logistics-an overview of aspects, issues, contributions and challenges, European Journal of Operational Research, 219(3), 671-679.

Demir, E., T. Bektaş, and G. Laporte (2014), A review of recent research on green road freight transportation, European Journal of Operational Research, 237(3), 775-793.

Disney, S. M., and D. R. Towill (2003), The effect of vendor managed inventory dynamics on the bullwhip effect in supply chains, International Journal of Production Economics, 85(2), 199-215.

Eleftheriadis, I., and E. Anagnostopoulou (2017), Measuring the level of corporate commitment regarding climate change strategies, International Journal of Climate Change Strategies and Management, 9(5), 626-644.

Estrada-Flores, S., and A. Eddy (2006), Thermal performance indicators for refrigerated road vehicles, Interna- 
tional Journal of Refrigeration, 29(6), 889-898.

Fatima, S. S., M. Wooldridge, and N. R. Jennings (2008), A linear approximation method for the shapley value, Artificial Intelligence, 172(14), 1673-1699.

Fawcett, S. E., M. W. McCarter, A. M. Fawcett, C. S. Webb, and G. M. Magnan (2015), Why supply chain collaboration fails: the socio-structural view of resistance to relational strategies, Supply Chain Management: An International Journal, 20(6), 648-663.

Frisk, M., M. Cöthe-Lundgren, K. Jörnsten, and M. Rönnqvist (2010), Cost allocation in collaborative forest transportation, European Journal of Operational Research, 205(2), 448-458.

Garlov, R., V. Saveliev, K. Gavrylov, L. Golovin, and H. Pedolsky (2002), Refrigeration of a food transport vehicle utilizing liquid nitrogen, US Patent 6,345,509.

Garnett, T. (2008), Cooking up a storm, Food, greenhouse gas emissions and our changing climate. Guildford, UK: Food Climate Research Network, Centre for Environmental Strategy, University of Surrey.

Glavič, P., and R. Lukman (2007), Review of sustainability terms and their definitions, Journal of Cleaner Production, 15(18), 1875-1885.

Gonzalez-Feliu, J., C. Peris-Pla, and D. Rakotonarivo (2010), Simulation and optimization methods for logistics pooling in the outbound supply chain, in Third International Conference on Value Chain Sustainability." Towards a Sustainable Development and Corporate Social Responsibility Strategies in the 21st Century Clobal Market, pp. 394 401.

Guajardo, M. (2018), Environmental benefits of collaboration and allocation of emissions in road freight transportation, in Zeimpekis V., Aktas E., Bourlakis M., Minis I. (eds) Sustainable Freight Transport, vol. 63, Springer, Cham.

Guajardo, M., and M. Rönnqvist (2016), A review on cost allocation methods in collaborative transportation, International Transactions in Operational Research, 23(3), 371-392.

Cuajardo, M., M. Rönnqvist, P. Flisberg, and M. Frisk (2018), Collaborative transportation with overlapping coalitions, European Journal of Operational Research.

Hariga, M., R. As'ad, and A. Shamayleh (2017), Integrated economic and environmental models for a multi stage cold supply chain under carbon tax regulation, Journal of Cleaner Production, 166, 1357-1371.

Hsu, C.-I., S.-F. Hung, and H.-C. Li (2007), Vehicle routing problem with time-windows for perishable food delivery, Journal of Food Engineering, 80(2), 465-475.

Huppes, G., and M. Ishikawa (2005), A framework for quantified eco-efficiency analysis, Journal of Industrial Ecology, 9(4), 25-41.

Jabali, O., T. Van Woensel, and A. De Kok (2012), Analysis of travel times and $\mathrm{CO}_{2}$ emissions in time-dependent vehicle routing, Production and Operations Management, 21(6), 1060-1074.

Jabir, E., V. V. Panicker, and R. Sridharan (2017), Design and development of a hybrid ant colony-variable neighbourhood search algorithm for a multi-depot green vehicle routing problem, Transportation Research Part $D$ : Transport and Environment, 57, 422-457.

Jaehn, F. (2016), Sustainable operations, European Journal of Operational Research, 253(2), 243-264.

James, S., and C. James (2010), The food cold-chain and climate change, Food Research International, 43(7), 1944 1956.

James, S., M. Swain, T. Brown, J. Evans, S. Tassou, Y. Ge, I. Eames, J. Missenden, G. Maidment, and D. Baglee (2009), Improving the energy efficiency of food refrigeration operations, in Proceedings of the Institute of Refrigeration, vol. 5.

James, S. J., C. James, and J. A. Evans (2006), Modelling of food transportation systems-a review, International Journal of Refrigeration, 29(6), 947-957.

Jonkman, J., A. Kanellopoulos, and J. M. Bloemhof (2019), Designing an eco-efficient biomass-based supply chain using a multi-actor optimisation model, Journal of Cleaner Production, 210, 1065-1075.

Kara, I., B. Y. Kara, and M. K. Yetis (2007), Energy minimizing vehicle routing problem, in International Conference on Combinatorial Optimization and Applications, pp. 62-71, Springer.

Kellner, F., and A. Otto (2012), Allocating $\mathrm{CO}_{2}$ emissions to shipments in road freight transportation, Journal of Management Control, 22(4), 451-479.

Ketzenberg, M., J. Bloemhof, and G. Gaukler (2015), Managing perishables with time and temperature history, Production and Operations Management, 24(1), 54-70. 
Koç, Ç., T. Bektaş, O. Jabali, and G. Laporte (2016), The impact of depot location, fleet composition and routing on emissions in city logistics, Transportation Research Part B: Methodological, 84, 81-102.

Koehler, J., W. J. Tegethoff, D. Westphalen, and M. Sonnekalb (1997), Absorption refrigeration system for mobile applications utilizing exhaust gasesabgasbetriebene absorptionskälteanlage für transportkühlung, Heat and Mass Transfer, 32(5), 333-340.

Kondjoyan, A. (2006), A review on surface heat and mass transfer coefficients during air chilling and storage of food products, International Journal of Refrigeration, 29(6), 863-875.

Kreutzberger, E. (2010), Lowest cost intermodal rail freight transport bundling networks: conceptual structuring and identification, European Journal of Transport and Infrastructure Research, 10(2).

Kruize, J. W., J. Wolfert, H. Scholten, C. Verdouw, A. Kassahun, and A. J. Beulens (2016), A reference architecture for farm software ecosystems, Computers and Electronics in Agriculture, 125, 12-28.

Kumar, S., H.-T. Kwon, K.-H. Choi, W. Lim, J. H. Cho, K. Tak, and I. Moon (2011), Lng: An eco-friendly cryogenic fuel for sustainable development, Applied energy, 88(12), 4264-4273.

Lin, C., K. L. Choy, G. T. Ho, S. H. Chung, and H. Lam (2014), Survey of green vehicle routing problem: past and future trends, Expert Systems with Applications, 41(4), 1118-1138.

Lozano, S., P. Moreno, B. Adenso-Díaz, and E. Algaba (2013), Cooperative game theory approach to allocating benefits of horizontal cooperation, European Journal of Operational Research, 229(2), 444-452.

Makaci, M., P. Reaidy, K. Evrard-Samuel, V. Botta-Cenoulaz, and T. Monteiro (2017), Pooled warehouse management: An empirical study, Computers \& Industrial Engineering, 112, 526-536.

Mavrotas, G. (2009), Effective implementation of the $\varepsilon$-constraint method in multi-objective mathematical programming problems, Applied Mathematics and Computation, 213(2), 455-465.

McKinnon, A., Y. Ge, and D. Leuchars (2003), Analysis of transport efficiency in the uk food supply chain, Logistics Research Centre Heriot-Watt University, Edinburgh.

McKinnon, A. C. (2016), Freight transport deceleration: Its possible contribution to the decarbonisation of logistics, Transport Reviews, 36(4), 418-436.

Mercier, S., S. Villeneuve, M. Mondor, and I. Uysal (2017), Time-temperature management along the food cold chain: A review of recent developments, Comprehensive Reviews in Food Science and Food Safety, 16(4), 647-667.

Miller, C. E., A. W. Tucker, and R. A. Zemlin (1960), Integer programming formulation of traveling salesman problems, Journal of the Association for Computing Machinery, 7(4), 326-329.

Mishra, A., R. L. Buchanan, D. W. Schaffner, and A. K. Pradhan (2016), Cost, quality, and safety: A nonlinear programming approach to optimize the temperature during supply chain of leafy greens, LWT-Food Science and Technology, 73, 412-418.

Mittal, A., C. Krejci, and T. Craven (2018), Logistics best practices for regional food systems: A review, Sustainability, 10(1), 168

Molina, J. C., I. Eguia, J. Racero, and F. Guerrero (2014), Multi-objective vehicle routing problem with cost and emission functions, Procedia-Social and Behavioral Sciences, 160, 254-263.

Morgan, R. M., and S. D. Hunt (1994), The commitment-trust theory of relationship marketing, Journal of marketing, 58(3), 20-38.

Naber, S., D. de Ree, R. Spliet, and W. van den Heuvel (2015), Allocating $\mathrm{CO}_{2}$ emission to customers on a distribution route, Omega, 54, 191-199.

Nagarajan, M., and G. Sošić (2008), Game-theoretic analysis of cooperation among supply chain agents: Review and extensions, European Journal of Operational Research, 187(3), 719-745.

Neto, J. Q. F., G. Walther, J. Bloemhof, J. Van Nunen, and T. Spengler (2009), A methodology for assessing ecoefficiency in logistics networks, European Journal of Operational Research, 193(3), 670-682.

Niknamfar, A. H., and S. T. A. Niaki (2016), Fair profit contract for a carrier collaboration framework in a green hub network under soft time-windows: Dual lexicographic max-min approach, Transportation Research Part E: Logistics and Transportation Review, 91, 129-151.

Osvald, A., and L. Z. Stirn (2008), A vehicle routing algorithm for the distribution of fresh vegetables and similar perishable food, Journal of Food Engineering, 85(2), 285-295.

Özener, O., Ö. Ergun, and M. Savelsbergh (2013), Allocating cost of service to customers in inventory routing, Operations Research, 61(1), 112-125. 
Özener, O. Ö. (2014), Developing a collaborative planning framework for sustainable transportation, Mathematical Problems in Engineering, 2014.

Paddeu, D., G. Parkhurst, G. Fancello, P. Fadda, and M. Ricci (2018), Multi-stakeholder collaboration in urban freight consolidation schemes: drivers and barriers to implementation, Transport, 33(4), 913-929.

Palhazi Cuervo, D., C. Vanovermeire, and K. Sörensen (2016), Determining collaborative profits in coalitions formed by two partners with varying characteristics, Transportation Research Part C: Emerging Technologies, 70, 171-184.

Palmer, A. (2007), The development of an integrated routing and carbon dioxide emissions model for goods vehicles. PhD thesis cranfield university, Ph.D. thesis, Cranfield University.

Pelletier, S., O. Jabali, and G. Laporte (2016), 50th anniversary invited article-goods distribution with electric vehicles: review and research perspectives, Transportation Science, 50(1), 3-22.

Pérez-Bernabeu, E., A. A. Juan, J. Faulin, and B. B. Barrios (2015), Horizontal cooperation in road transportation: a case illustrating savings in distances and greenhouse gas emissions, International Transactions in Operational Research, 22(3), 585-606.

Pettit, T. J., J. Fiksel, and K. L. Croxton (2010), Ensuring supply chain resilience: development of a conceptual framework, Journal of Business Logistics, 31(1), 1-21.

Pham, Q. T. (2006), Modelling heat and mass transfer in frozen foods: a review, International Journal of Refrigeration, 29(6), 876-888.

Quintero-Araujo, C. L., A. Gruler, A. A. Juan, J. de Armas, and H. Ramalhinho (2017), Using simheuristics to promote horizontal collaboration in stochastic city logistics, Progress in Artificial Intelligence, 6(4), 275-284.

Ramanathan, U., Y. Bentley, and G. Pang (2014), The role of collaboration in the UK green supply chains: an exploratory study of the perspectives of suppliers, logistics and retailers, Journal of Cleaner Production, 70, 231-241.

Reijnders, L. (1998), The factor x debate: setting targets for eco-efficiency, Journal of Industrial Ecology, 2(1), 13-22.

Rogelj, J., M. Den Elzen, N. Höhne, T. Fransen, H. Fekete, H. Winkler, R. Schaeffer, F. Sha, K. Riahi, and M. Meinshausen (2016), Paris agreement climate proposals need a boost to keep warming well below $2^{\circ} \mathrm{C}$, Nature, 534(7609), 631.

Romero, C., and T. Rehman (2003), Multiple Criteria Analysis for Agricultural Decisions, vol. 11, Elsevier.

Rong, A., R. Akkerman, and M. Grunow (2011), An optimization approach for managing fresh food quality throughout the supply chain, International Journal of Production Economics, 131(1), 421-429.

Sanchez, M., L. Pradenas, J.-C. Deschamps, and V. Parada (2016), Reducing the carbon footprint in a vehicle routing problem by pooling resources from different companies, NETNOMICS: Economic Research and Electronic Networking, 17(1), 29-45.

Scholten, K., and S. Schilder (2015), The role of collaboration in supply chain resilience, Supply Chain Management: An International Journal, 20(4), 471-484.

Shapley, L. S. (1953), A value for n-person games, Contributions to the Theory of Games, 2(28), 307-317.

Simatupang, T. M., and R. Sridharan (2002), The collaborative supply chain, The International Journal of Logistics Management, 13(1), 15-30.

Solomon, M. M. (1987), Algorithms for the vehicle routing and scheduling problems with time window constraints, Operations Research, 35(2), 254-265.

Song, B. D., and Y. D. Ko (2016), A vehicle routing problem of both refrigerated-and general-type vehicles for perishable food products delivery, Journal of Food Engineering, 169, 61-71.

Soysal, M., J. M. Bloemhof-Ruwaard, R. Haijema, and J. C. Van der Vorst (2015), Modeling an inventory routing problem for perishable products with environmental considerations and demand uncertainty, International Journal of Production Economics, 164, 118-133.

Soysal, M., J. M. Bloemhof-Ruwaard, R. Haijema, and J. G. Van der Vorst (2018), Modeling a green inventory routing problem for perishable products with horizontal collaboration, Computers \& Operations Research, 89 , 168-182.

Spence, S. W., W. J. Doran, and D. W. Artt (2004), Design, construction and testing of an air-cycle refrigeration system for road transport, International Journal of Refrigeration, 27(5), 503-510.

Stellingwerf, H. M., A. Kanellopoulos, J. G. A. J. van der Vorst, and J. M. Bloemhof (2018a), Reducing $\mathrm{CO}_{2}$ emissions in temperature-controlled road transportation using the LDVRP model, Transportation Research Part D: 
Transport and Environment, 58, 80-93.

Stellingwerf, H. M., G. Laporte, F. C. Cruijssen, A. Kanellopoulos, and J. M. Bloemhof (2018b), Quantifying the environmental and economic benefits of cooperation: A case study in temperature-controlled food logistics, Transportation Research Part D: Transport and Environment, 65, 178-193.

Tassou, S., G. De-Lille, and Y. Ge (2009), Food transport refrigeration-approaches to reduce energy consumption and environmental impacts of road transport, Applied Thermal Engineering, 29(8-9), 1467-1477.

Tijs, S. H., and T. S. H. Driessen (1986), Game theory and cost allocation problems, Management Science, 32(8), 1015-1028.

Toth, P., and D. Vigo (2002), Models, relaxations and exact approaches for the capacitated vehicle routing problem, Discrete Applied Mathematics, 123(1-3), 487-512.

Tsironi, T., E. Dermesonlouoglou, M. Giannoglou, E. Gogou, G. Katsaros, and P. Taoukis (2017), Shelf-life prediction models for ready-to-eat fresh cut salads: Testing in real cold chain, International Journal of Food Microbiology, 240, 131-140.

Tso, C., S. Yu, H. Poh, and P. Jolly (2002), Experimental study on the heat and mass transfer characteristics in a refrigerated truck, International Journal of Refrigeration, 25(3), 340-350.

Van Boekel, M. A. (2008), Kinetic modeling of food quality: a critical review, Comprehensive Reviews in Food Science and Food Safety, 7(1), 144-158.

Van Der Vorst, J. G., S.-O. Tromp, and D.-J. v. d. Zee (2009), Simulation modelling for food supply chain redesign; integrated decision making on product quality, sustainability and logistics, International Journal of Production Research, 47(23), 6611-6631.

Vanovermeire, C., and K. Sörensen (2014), Measuring and rewarding flexibility in collaborative distribution, including two-partner coalitions, European Journal of Operational Research, 239(1), 157-165.

Vanovermeire, C., K. Sörensen, A. Van Breedam, B. Vannieuwenhuyse, and S. Verstrepen (2014), Horizontal logistics collaboration: decreasing costs through flexibility and an adequate cost allocation strategy, International Journal of Logistics Research and Applications, 17(4), 339-355.

Vrat, P., R. Cupta, A. Bhatnagar, D. K. Pathak, and V. Fulzele (2018), Literature review analytics on sustainable cold-chain for perishable food products: research trends and future directions, OPSEARCH, pp. 1-27.

Wang, Y., X. Ma, Z. Li, Y. Liu, M. Xu, and Y. Wang (2017), Profit distribution in collaborative multiple centers vehicle routing problem, Journal ofCleaner Production, 144, 203-219.

Xiao, Y., and A. Konak (2015), A simulating annealing algorithm to solve the green vehicle routing \& scheduling problem with hierarchical objectives and weighted tardiness, Applied Soft Computing, 34, 372-388.

Xiao, Y., Q. Zhao, I. Kaku, and Y. Xu (2012), Development of a fuel consumption optimization model for the capacitated vehicle routing problem, Computers \& Operations Research, 39(7), 1419-1431.

Zachariadis, E. E., C. D. Tarantilis, and C. T. Kiranoudis (2015), The load-dependent vehicle routing problem and its pick-up and delivery extension, Transportation Research Part B: Methodological, 71, 158-181.

Zhang, Y., and X. Chen (2014), An optimization model for the vehicle routing problem in multi-product frozen food delivery, Journal of Applied Research and Technology, 12(2), 239-250.

Zhu, W., M. P. Nowak, and S. O. Erikstad (2016), Emission allocation issues in repositioning transportation, International Journal of Sustainable Transportation, 10(4), 365-375. 


\section{Statement of authorship contribution}

An overview of the contribution from co-authors for the other chapters is provided below.

$$
\begin{aligned}
\mathrm{AK} & =\text { A. Kanellopoulos } \\
\mathrm{BB} & =\text { B. Behdani } \\
\mathrm{FC} & =\text { F.C.A.M. Cruijssen } \\
\mathrm{CL} & =\text { G. Laporte } \\
\mathrm{HS} & =\text { H.M. Stellingwerf } \\
\mathrm{JB} & =\text { J.M. Bloemhof } \\
\mathrm{JV} & =\text { J.C.A.J. van der Vorst } \\
\mathrm{LC} & =\text { L.H.C. Groeneveld }
\end{aligned}
$$

\section{Chapter 1}

Conception or design of the work: HS, AK, JB, JV

Data collection: -

Data analysis and interpretation:

Drafting the article: HS

Critical revision of the article: AK, JB, JV

Approval of the version to be published: HS, AK, JB, JV

\section{Chapter 2}

Conception or design of the work: HS, AK, JV, JB

Data collection: HS

Data analysis and interpretation: HS in consultation with AK, JV, JB

Drafting the article: HS

Critical revision of the article: AK, JB

Approval of the version to be published: HS, AK, JV, JB

\section{Chapter 3}

Conception or design of the work: HS, LC, BB

Data collection: HS

Data analysis and interpretation: $\mathrm{HS}$ and $\mathrm{LG}$ in consultation with $\mathrm{AK}, \mathrm{GL}, \mathrm{AK}, \mathrm{BB}$

Drafting the article: HS

Critical revision of the article: $L G, A K, B B, J B, G L$

Approval of the version to be published: HS, LC, GL, AK, JB, BB 


\section{Chapter 4}

Conception or design of the work: HS, GL, AK

Data collection: HS, FC

Data analysis and interpretation: $\mathrm{HS}$ in consultation with $\mathrm{GL}, \mathrm{AK}, \mathrm{FC}$, JB

Drafting the article: HS

Critical revision of the article: CL, AK

Approval of the version to be published: $\mathrm{HS}, \mathrm{GL}, \mathrm{AK}, \mathrm{FC}, \mathrm{JB}$

\section{Chapter 5}

Conception or design of the work: HS, AK, FC, JB

Data collection: HS

Data analysis and interpretation: $\mathrm{HS}$ in consultation with $\mathrm{AK}, \mathrm{FC}, \mathrm{JB}$

Drafting the article: HS

Critical revision of the article: AK, JB

Approval of the version to be published: $\mathrm{HS}, \mathrm{AK}, \mathrm{FC}, \mathrm{JB}$

\section{Chapter 6}

Conception or design of the work: HS

Data collection: -

Data analysis and interpretation: -

Drafting the chapter: $\mathrm{HS}$

Critical revision of the chapter: AK, JV, JB

Approval of the version to be published: : HS, AK, JV, JB 



\section{Summary}

Road transportation generates significant costs for firms that deliver and collect products. Next to that, it results in emissions. Food supply chains cause additional emissions because of the extra energy needed to guarantee product quality. Moreover, current transportation systems are inefficient since the available capacity is not optimally used. Cooperation between food supply chain actors could provide opportunities to reduce costs and emissions and improve eco-efficiency, which is defined as solutions for which it is impossible to improve the environmental objective without worsening the economic objective. In food supply chains, it is also important to guarantee food quality. However, reducing costs, emissions, and quality decay do not always go hand in hand, and trade-offs need to be made. Moreover, despite the benefits, companies hesitate to implement cooperation because it might bring advantages to competitors and they find it difficult to agree on gain sharing. To find out how cooperation can improve eco-efficiency in food logistics, we need decision support models that can capture these complexities. Therefore, the main research question that this thesis aims to answer is: Which decision support models can be used to design eco-efficient logistics cooperation in food supply chains?

All studies in this PhD thesis are based on a case study on a Dutch retail cooperative, where several smaller retail organisations cooperate by forming a buying organisation. By jointly purchasing their products, they can negotiate a lower unit price. The retailers currently hire different logistics service providers to pick up their orders from their shared distribution centre and bring them to their own distribution centre. From there, they distribute the products to their supermarket outlets. Currently, the retailers arrange their logistics individually, but they are considering to cooperate to reduce costs, emissions and quality decay.

In this thesis, the effects of different forms of logistics cooperation between food supply chain actors are analysed using existing optimisation models, which we extended to account for temperature control and food quality. Using these extended models, routing and inventory are optimised to minimise costs, emissions, and quality loss. Moreover, trade-offs between the objectives are established. Also, we proposed a method to divide cooperative gains, not only based on costs but also on emissions. This way, eco-efficient forms of logistics cooperation are rewarded and stimulated.

In Chapter 2, we extend the Load Dependent Vehicle Routing Model such that it accounts for the extra costs and emissions related to temperature control. We show that temperature control can significantly affect costs and emissions and thus the optimal routing. 
This extended model can be used to test the effect of new cooling technologies on the costs and emissions of routing.

In Chapter 3, we introduce the Quality Driven Vehicle Routing Problem. This problem is modelled and used to more realistically quantify how food degrades during distribution processes. We consider effects of outside temperature, door openings, and differences in optimal temperatures for different products. When transporting temperaturesensitive products, minimising quality loss results in multiple routes with less stops per route whereas minimising costs or emissions results in longer routes. The negative quality effects of multi-stop routes can be mitigated by adjusting driving speed, unloading rate, cooling rate, and by setting a quality threshold level.

In Chapter 4, we compare the effect of different forms of cooperation in temperaturecontrolled transportation on cost and emissions. Joint route planning (JRP, in which daily transport decisions are optimised cooperatively) is compared to vendor-managed inventory (VMI, in which multi-day routing and inventory decisions are optimised cooperatively) and to a non-cooperative scenario using vehicle routing and inventory routing models. In JRP, there is one optimal solution for minimising costs and for minimising emissions. For VMI however, additional savings in both objectives are obtained but there is a set of alternative eco-efficient solutions and partners need to choose which of those solutions (i.e. cooperative routing and inventory plans) they prefer. Also, in VMI there exists a trade-off between product age and emissions: less frequent inventory replenishment leads to reduced emissions but it also to a higher average product age.

In Chapter 5, we study how the monetary benefits of VMI can be allocated. We discuss that gain allocations should reflect both contributions to savings in costs and emissions. That way, gain allocation can be used to stimulate eco-efficient forms of cooperation. A green IRP model is used to quantify cooperative benefits and establish all possible ecoefficient cooperative solutions. For each solution, we allocate monetary benefits based on costs and emissions using the Shapley value. This approach results in cost savings for all partners that help reducing impacts.

In this thesis, we adjusted VRP and IRP models to account for temperature control and perishability. Using these OR models, we found that food logistics cooperation can result in significant economic and environmental benefits. The findings of all studies can be summarised in three main concluding statements: (i) temperature control influences costs and emissions of cooperative routes, and cooperative routing influences food quality. Therefore, these food specific aspects should be considered in cooperative logistics; (ii) dependent on the intensity of the cooperation, it can result either in one optimal solution for both costs and emissions, or a set of eco-efficient solutions; and (iii) to stimulate forms of cooperation that both reduce costs and emissions, we should allocate cooperative gains based on partner's contributions to both indicators. 


\section{Samenvatting}

Wegtransport is een grote kostenpost voor bedrijven die producten bezorgen en ophalen. Daarnaast veroorzaakt het uitstoot. Levensmiddelenketens zorgen daarbovenop nog voor extra uitstoot omdat ze energie gebruiken voor koeling om productkwaliteit te kunnen garanderen. Verder wordt in de huidige transportsystemen de capaciteit niet optimaal benut. Samenwerking tussen actoren in levensmiddelenketens kan mogelijkheden bieden om kosten en uitstoot te verminderen en eco-efficiëntie te verbeteren. Een situatie kan worden gezien als eco-efficiënt wanneer het niet mogelijk is verdere milieuwinst te behalen zonder er financieel op achteruit te gaan en andersom. In levensmiddelenketens is het daarnaast ook belangrijk om voedselkwaliteit te garanderen. Helaas gaan het verbeteren van kwaliteit en het verminderen van uitstoot en kosten niet altijd hand in hand en moeten er afwegingen gemaakt worden. Bedrijven hebben, ondanks de beschreven voordelen, vaak een aarzelende houding ten opzichte van samenwerking, omdat het ook voordelen kan brengen voor concurrenten en ze het eens moeten worden over het verdelen van de besparingen. Om erachter te komen hoe samenwerking eco-efficiëntie kan bevorderen, zijn beslissingsondersteunende modellen nodig die met dit soort complexiteit kunnen omgaan. Daarom is de hoofdvraag van deze dissertatie: Welke beslissingsondersteunende modellen kunnen gebruikt worden om eco-efficiënte vormen van samenwerking te ontwerpen in de voedsellogistiek?

De onderzoeken in deze dissertatie zijn gebaseerd op een studie bij een Nederlandse inkooporganisatie voor supermarkten. Hierin werken verschillende supermarktketens samen. Door samen in te kopen, kunnen ze beter onderhandelen over de productprijs. In de huidige situatie huren de supermarktketens verschillende logistieke dienstverleners in om hun bestellingen op te halen bij het gezamenlijke distributiecentrum: ze plannen hun logistiek dus individueel. Ze overwegen ook op het gebied van logistiek samen te werken om zo kosten, uitstoot en kwaliteitsvermindering tegen te gaan.

In deze dissertatie worden de effecten van verschillende vormen van logistieke samenwerking tussen actoren in de levensmiddelenketen geanalyseerd met bestaande optimalisatiemodellen die uitgebreid zijn om rekening te houden met het effect van gekoeld transport en het effect op voedselkwaliteit. Met deze uitgebreide modellen worden transport- en voorraadbeslissingen geoptimaliseerd om kosten, uitstoot en kwaliteitsvermindering te minimaliseren. Bovendien worden de modellen gebruikt om de afweging tussen de verschillende doelfuncties (zoals uitstootvermindering, kostenminimalisatie en kwaliteitsverbetering) in kaart te brengen. Ook wordt een methode geïntroduceerd om de kostenbesparingen als gevolg van samenwerking eerlijk te verdelen, gebaseerd op 
zowel kosten als emissies. Op deze manier worden eco-efficiënte vormen van logistieke samenwerking beloond en gestimuleerd.

In hoofdstuk 2 wordt het Load Dependent Vehicle Routing Model uitgebreid zodat rekening wordt gehouden met kosten en uitstoot gerelateerd aan het koelen van vrachtwagens. Uit de resultaten blijkt dat koeling leidt tot een significante verhoging van kosten en uitstoot en dat het daarom nodig is de standaard routeplanning aan te passen. Dit model kan worden gebruikt om de effecten van nieuwe koeltechnieken op kosten en emissies in kaart te brengen.

In hoofdstuk 3 wordt het Quality Driven Vehicle Routing Problem geïntroduceerd. Dit probleem wordt gemodelleerd en gebruikt om realistischer te kwantificeren hoe voedselkwaliteit verandert gedurende het distributieproces. Hierbij wordt rekening gehouden met de effecten van de buitentemperatuur, het openen van de deur van de wagen en verschillen in de optimale temperatuur van verschillende producten die samen vervoerd worden. In dit hoofdstuk blijkt dat het minimaliseren van kwaliteitsverlies in meerdere, kortere routes resulteert wanneer temperatuurgevoelige producten vervoerd worden. Het minimaliseren van kosten en emissies resulteert daarentegen in langere routes. De negatieve kwaliteitseffecten van routes met veel tussenstops worden verminderd door het aanpassen van rijsnelheid, uitlaadsnelheid, koelefficiëntie en door een minimumkwaliteit in te stellen voor alle klanten.

In hoofdstuk 4 worden de effecten van verschillende vormen van logistieke samenwerking in koeltransport vergeleken op basis van kosten en uitstoot met behulp van een inventory routing problem (IRP). Joint route planning (JRP, waarbij dagelijkse transportbeslissingen samen worden geoptimaliseerd) wordt vergeleken met vendor-managed inventory (VMI, waarbij meerdaagse transport-en opslagbeslissingen samen worden genomen) en met een scenario waarin transport en opslag individueel worden geoptimaliseerd. Met]RP is er één optimale oplossing wat betreft kost- en uitstootminimalisatie. Met VMI kunnen extra besparingen in kosten en uitstoot worden behaald maar is er een set van alternatieve eco-efficiënte oplossingen. De partners moeten in dit geval dus kiezen voor welke oplossing (m.a.w. welk plan voor transport en opslag) ze een voorkeur hebben. Daarnaast moet er bij VMI samenwerking een afweging worden gemaakt tussen de versheid van een product en de uitstoot: als de voorraad mindervaak wordt aangevuld, wordt uitstoot bespaard, maar gaat de gemiddelde versheid van de producten naar beneden.

In hoofdsuk 5 wordt er gekeken hoe de financiële voordelen van VMI verdeeld kunnen worden over de partners. De verdeling van de voordelen moet gebaseerd worden op de bijdragen van de verschillende partners aan het besparen van kosten, maar ook op de bijdragen van de partners aan het besparen van uitstoot. Op deze manier kan het verdelen van de baten van samenwerking zorgen dat eco-efficiënte vormen van samenwerking gestimuleerd worden. Een green inventory routing problem (GIRP) model wordt gebruikt om de gezamenlijke voordelen te kwantificeren en alle mogelijke eco-efficiënte oplossingen in kaart te brengen. Voor elke oplossing worden de financiële voordelen verdeeld gebaseerd op kosten en uitstoot met behulp van zogenaamde Shapley waarde. 
Deze aanpak resulteert in kostenbesparingen voor alle partners die helpen om de gezamenlijke impact te verminderen.

In deze dissertatie zijn VRP en IRP modellen aangepast zodat ze het effect van koeling en de effecten op kwaliteit kunnen meten. Met behulp van deze modellen blijkt dat samenwerking in de voedsellogistiek zorgt voor significante economische besparingen en ook milieuvoordelen biedt. De bevindingen van deze studies kunnen worden samengevat in drie punten: (i) transportkoeling beïnvloedt kosten en uitstoot van gezamenlijke routes en het gezamenlijk organiseren van transport beïnvloedt voedselkwaliteit. Daarom is het belangrijk om rekening te houden met voedselspecifieke eigenschappen bij samenwerking in de logistiek; (ii) afhankelijk van de vorm van logistieke samenwerking, kan het zorgen voor óf één optimale oplossing voor zowel kosten als uitstoot óf een set van eco-efficiënte oplossingen; en (iii) om vormen van samenwerking te stimuleren die zowel kosten als uitstoot verminderen, moeten gezamenlijke verdiensten worden gebaseerd op bijdragen van partners op beide gebieden. 


\section{Acknowledgements}

Only in the end of my Master's, I realised that it would maybe be fun to pursue a PhD degree. When the possibility of doing a PhD in food logistics presented itself, I decided to go for it. I knew about food but logistics was a nearly new field of study, so in the beginning it felt much like 'trying something' instead of 'working on something'. Luckily I had supervisors to help me succeed. Jacqueline, you have been there and supported me during the whole PhD trajectory, also when you were limited by your health. The last four years have without a doubt been more challenging for you than for me, but I could always count on your feedback. Jack, we were mainly involved in the beginning to help me start up and in the end to help me finish and I greatly appreciate that. It was very helpful that you kept the bigger picture in mind when I wanted to focus too much on tiny details. When Jack had to take a step back from the supervision, Argyris joined the team. Argyris, I am super happy that you've been my supervisor and friend and that I could always walk in your office to disturb you for a chat or to go for a run.

Next to my supervisors, I've also learnt a lot from working with other co-authors: Behzad, Frans, Gilbert, and Luuk. You've all helped to generate ideas, models, improve my writing, and to publish. People see a PhD project as lonely work but l've really enjoyed working together with you. I've also really enjoyed being around the people on the $6^{\text {th }}$ floor. Especially the ones in room 6029, you have been a great source of inspiration and distraction.

When I started my PhD, Peter went from being my friend to being my boyfriend, and now, we have a 'samenlevingscontract' and a house together. We also passed the official milestone of having a cat, which some people already see as a reason to call themselves parents, but soon enough, we'll really be able to call ourselves parents. Peter, you've been the greatest support during these years and whereas my PhD stops, I'm very sure that we will keep having adventures together.

Then, there were also other people that I've enjoyed being around a lot, the Bonzais, the Foodies, the Pomonians, the Montréal friends, the $8 \mathrm{c}$ gangsters and the $7 \mathrm{c}$ girls. And last, but definitely not least, my family and their 'aanhang' (the Dutch language is so poetic): thanks for your support!

Heleen 


\section{Curriculum Vitae}

Helena Margaretha Stellingwerf

July 31,1990 , Rheden

2002-2008 Pre-university education (VWO), Christelijk Lyceum Delft

Profile Nature and Health

2008-2011 BSc Food Technology, Wageningen University

Minor Marine Living Resources

2012-2014 MSc Food Technology, Wageningen University

Specialisation Sustainable Food Process Engineering

Internship at WUR Chile, Santiago de Chile

2015-2019 PhD candidate, Wageningen University

Chair Group Operations Research and Logistcs

Including four-month visit to the Haute Etudes Commerciales (HEC)

Montréal, Canada 


\section{List of publications}

\section{Peer-reviewed publications}

H.M. Stellingwerf, A. Kanellopoulos, J.G.A.J. van der Vorst, and J.M. Bloemhof (2018), Reducing $\mathrm{CO}_{2}$ emissions in temperature-controlled road transportation using the LDVRP model, Transportation Research part D: Transport and Environment, 58, 80-93, doi: https://doi.org/10.1016/j.trd.2017.11.008.

H.M. Stellingwerf, G. Laporte, F.C.A.M. Cruijssen, A. Kanellopoulos, and J.M. Bloemhof (2018), Quantifying the environmental and economic benefits of cooperation: A case study in temperature-controlled food logistics, Transportation Research part D: Transport and Environment, 65, 178-193, doi: https://doi.org/10.1016/j.trd. 2018.08.010

H.M. Stellingwerf, A. Kanellopoulos, F.C.A.M. Cruijssen and J.M. Bloemhof (2019), Fair gain allocation in ecoefficient vendor-managed inventory cooperation, Journal of Cleaner Production, accepted for publication.

H.M. Stellingwerf, L.H.C. Groeneveld, G. Laporte, A. Kanellopoulos, J.M. Bloemhof, and B. Behdani (2019), The quality-driven vehicle routing problem: Model and application to a case of cooperative logistics, under review.

\section{Book chapter}

H.M. Stellingwerf, A. Kanellopoulos, and J.M. Bloemhof (2019), Sustainable Food Supply Chains: Planning, Design, and Control through Interdisciplinary Methodologies (Eds: Riccardo Accorsi Riccardo Manzini), Ch. 11: Using vehicle routing models to improve sustainability of temperature-controlled food chains, Elsevier, Amsterdam, The Netherlands. 
Helena Margaretha Stellingwerf

Wageningen School of Social Sciences (WASS)

Completed Training and Supervision Plan

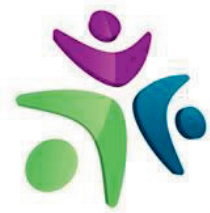

Wageningen School

of Social Sciences

\begin{tabular}{|c|c|c|c|}
\hline Name of the learning activity & Department/Institute & Year & ECTS* \\
\hline \multicolumn{4}{|l|}{ A) Project related competences } \\
\hline Operations Research and Logistics (ORL-30806) & WUR & 2015 & 6 \\
\hline Transport Logistics Modelling & GP-OML & 2015 & 4 \\
\hline OR-games & LNMB & 2015 & 4 \\
\hline Predicting Food Quality (FQD-31306) & WUR & 2016 & 6 \\
\hline Quantitative modelling and analysis of Supply Chains & GP-OML & 2016 & 1 \\
\hline EURO PhD Summer School on Sustainable Supply chains & $\begin{array}{l}\text { EURO working group sustainable supply } \\
\text { chains }\end{array}$ & 2016 & 5 \\
\hline Data Management Planning & WGS & 2016 & 0.4 \\
\hline Research visit with professor Gilbert Laporte (4 months) & $\begin{array}{l}\text { École des Hautes Études Commerciales, } \\
\text { Montréal, Canada }\end{array}$ & 2017 & 0.5 \\
\hline Supply Chain Guru X Foundations in Network Optimization & Llamasoft & 2018 & 0.5 \\
\hline Introduction in R & WASS & 2018 & 2 \\
\hline \multicolumn{4}{|l|}{ B) General research related competences } \\
\hline WASS introduction course & WASS & 2015 & 1 \\
\hline Essentials of Scientific Writing and Presenting & WGS & 2015 & 1.2 \\
\hline $\begin{array}{l}\text { Logistics collaboration for sustainability performance } \\
\text { improvement in the Dutch food retail sector' and attendance } \\
\text { doctoral workshop }\end{array}$ & $\begin{array}{l}\text { Logistics Research Network Conference, } \\
\text { Derby, UK }\end{array}$ & 2015 & 1 \\
\hline Scientific Writing & WGS & 2016 & 1.8 \\
\hline Project \& time management & WGS & 2016 & 1.5 \\
\hline $\begin{array}{l}\text { 'Allocating collaborative gains based on both economic and } \\
\text { environmental contributions: towards sustainable logistics' }\end{array}$ & $\begin{array}{l}\text { European Conference on Operations } \\
\text { Research, Poznan, Poland }\end{array}$ & 2016 & 0.5 \\
\hline $\begin{array}{l}\text { 'Designing eco-efficient transportation in fresh food supply } \\
\text { chains using load and temperature dependent VRPs' }\end{array}$ & TRAll conference, Utrecht & 2016 & 0.5 \\
\hline 'Towards sustainable collaborative logistics' & $\begin{array}{l}\text { Erasmus Research Institute of Management, } \\
\text { Rotterdam }\end{array}$ & 2017 & 0.5 \\
\hline $\begin{array}{l}\text { 'Reducing fuel consumption and emissions in temperature } \\
\text { controlled road transportation' }\end{array}$ & $\begin{array}{l}\text { International Federation of Operational } \\
\text { Research Societies, Quebec, Canada }\end{array}$ & 2017 & 0.5 \\
\hline $\begin{array}{l}\text { Reviewing scientific papers for 'Computers and Electronics in } \\
\text { Agriculture', 'Annals of Operations Research', 'International } \\
\text { Journal of Production Economics' and 'Transportation } \\
\text { Research part D: Transport and Environment' }\end{array}$ & Elsevier and Springer & $2015-$ & 0.5 \\
\hline \multicolumn{4}{|l|}{ C) Career related competences/personal development } \\
\hline Competence Assessment & WGS & 2015 & 0.3 \\
\hline Research Proposal writing & WASS & 2016 & 4 \\
\hline $\begin{array}{l}\text { Participating in supervising MSc and BSc students and } \\
\text { assisting in ORL courses: advanced supply chain } \\
\text { management, decision science II, sustainability in food } \\
\text { chains, bio-based logistics }\end{array}$ & WUR & $\begin{array}{l}2015- \\
2019\end{array}$ & 2 \\
\hline Total & & & 44.7 \\
\hline
\end{tabular}


The work presented in this thesis is part of the research programme CapsLog, which is (partly) financed by the Netherlands Organisation for Scientific Research (NWO), and by the Canadian Natural Sciences and Engineering Research Council under grant 2015-01689. This support is gratefully acknowledged.

Cover by Proefschriftmaken.nl 



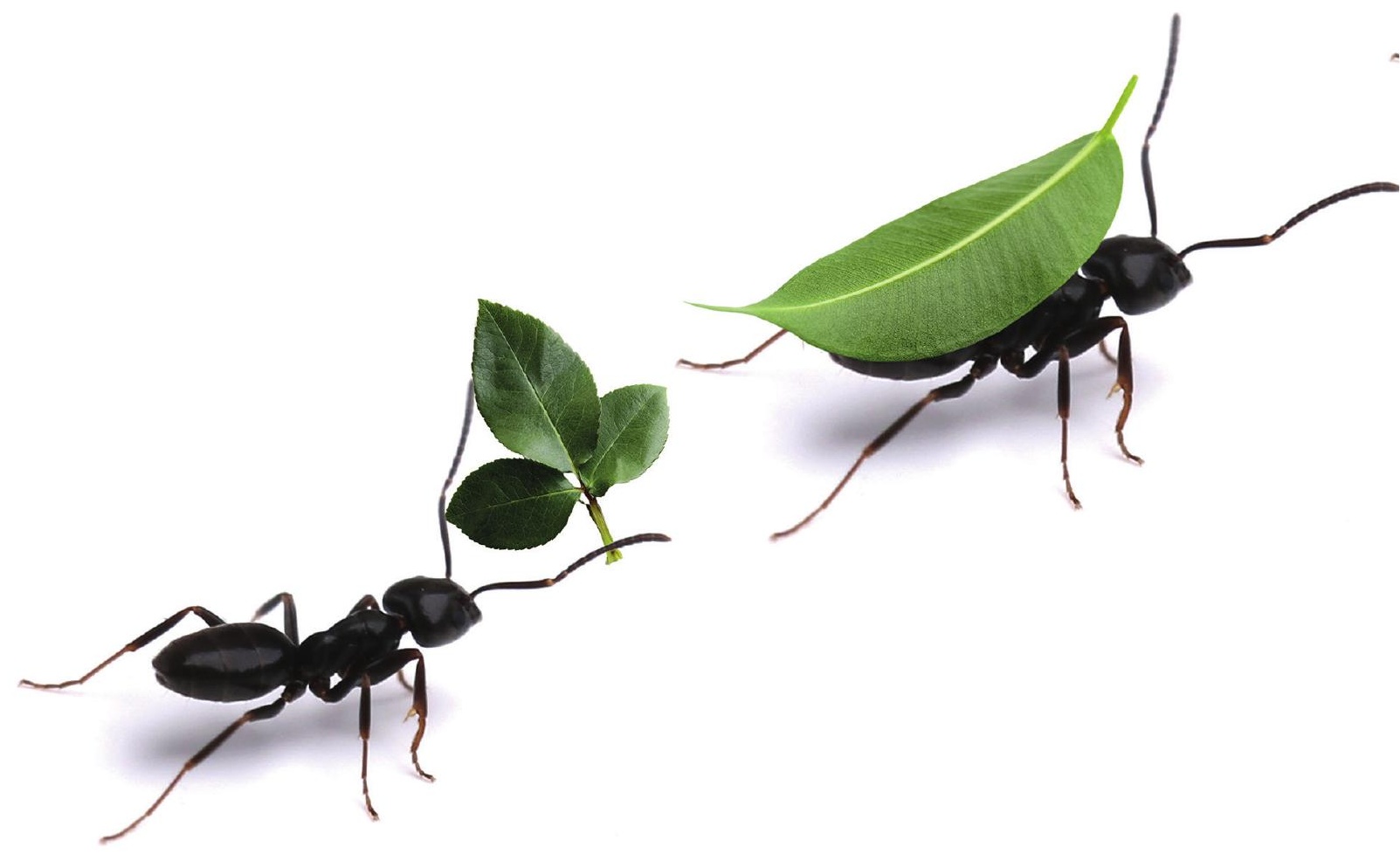

